\author{
Universidade de São Paulo \\ Instituto de Astronomia, Geofísica e Ciências Atmosféricas
}

Departamento de Astronomia

Pedro E. M. de Almeida

\title{
Cosmologias com Decaimento do Vácuo e K-essência
}

São Paulo 

Pedro E. M. de Almeida

\section{Cosmologias com Decaimento do Vácuo e K-essência}

Dissertação apresentada ao Departamento de Astronomia do Instituto de Astronomia, Geofísica e Ciências Atmosféricas da Universidade de São Paulo como requisito parcial para a obtenção do título de Mestre em Ciências.

Versão Corrigida. O original encontra-se disponível na Unidade.

Área de Concentração: Astronomia Orientador: Prof. Dr. José Ademir Sales de Lima

São Paulo 

Dedico esse trabalho à toda minha família; em especial aos meus pais Maria Angélica e João Mendonça e aos meus irmãos Heloísa e Maurício. 



\section{Agradecimentos}

Gostaria de agradecer primeiramente ao meu orientador, José Ademir Sales de Lima, pela oportunidade de formação cultural, profissional e pessoal, por todas as discussões e elucidações das minhas dúvidas e, em especial, por ter me aberto as portas para o mundo da pesquisa;

Aos meus pais, João e Maria Angélica, que me proporcionaram toda a condição e apoio para que esse trabalho se realizasse; à toda minha família, pelo carinho, sempre presente mesmo quando distante e, em especial, ao meu primo Getúlio, por ter aberto às portas de sua casa para mim, quando cheguei em São Paulo;

Aos meus colegas de república, João e Victor, e à minha namorada Loreany, por fazerem de São Paulo a minha casa;

Aos professores e colegas do IAG, por todo o aprendizado e por contribuírem para a expansão dos meus horizontes e à todos os funcionários, por possibilitarem que esse ambiente funcione da melhor forma possível;

Aos colegas estudantes de cosmologia, Bruno, Henrique e José Júnior, por me fazerem companhia nas minhas dúvidas e na busca pelas respostas. Um agradecimento especial ao colega de instituto Sérgio, por ter compartilhado esses dois anos comigo e pela ajuda durante o processo de revisão dessa dissertação;

E, por fim, à CAPES, pelo apoio financeiro.

Esta tese/dissertação foi escrita em IATEX com a classe IAGTESE, para teses e dissertações do IAG. 

"O absurdo é resultado de uma razão lúcida que constata os seus limites."

Albert Camus 



\section{Resumo}

Nessa dissertação, faremos uma apresentação do modelo padrão da cosmologia, o $\Lambda$ CDM. Apresentaremos seu desenvolvimento e as evidências que o apontam como o melhor modelo para descrever o Universo observado até o momento. Em seguida, apresentaremos alguns dos principais problemas dentro desse modelo: o problema da coincidência cosmológica e o problema da constante cosmológica.

Com o objetivo de resolver esse problemas, foram propostos vários modelos alternativos na literatura. Nesse trabalho, nos concentraremos em uma classe de modelos com decaimento do vácuo, $\Lambda(\mathrm{t})$. Essa classe de modelos possui a vantagem de descrever uma transição exata e suave de uma fase De Sitter primordial instável para a fase da radiação padrão. Esse modelo é não-singular e livre de horizontes. Mostraremos também, como ele é capaz de aliviar os problemas do modelo padrão, mencionados acima.

Finalmente, na parte original da dissertação, utilizaremos uma teoria de campos escalares, denominada k-essência, para obter uma descrição de campo escalar do Universo primordial da classe de modelos com decaimento do vácuo. A formulação em termos de campos escalares pode auxiliar no estudo da fase inflacionária desse modelo.

Palavras-chave - cosmologia, decaimento do vácuo, constante cosmológica, k-essência 



\section{Abstract}

In this dissertation, we will make a presentation of the standard model of cosmology, the $\Lambda \mathrm{CDM}$. We will present its development and the evidence that point it as the best model to describe the Universe observed so far. We will, then, present some of the main problems within this model: the problem of cosmological coincidence and the problem of the cosmological constant.

In order to solve these problems, several alternative models have been proposed in the literature. In this work, we will focus on a class of models with vacuum decay, $\Lambda(\mathrm{t})$. This model has the advantage of describing an exact and smooth transition from an unstable primordial De Sitter phase to the standard radiation phase. This model is non-singular and free of horizons. We will also show how it is able to alleviate the problems of the standard model, mentioned above.

Finally, in the original section of the dissertation, we will use a scalar field theory, dubbed k-essence, to obtain a scalar field description of the vacuum decay model primordial Universe. The formulation in terms of scalar fields can assist in the study of the inflationary phase of this model.

Keywords - cosmology, vacuum decay, cosmological constant, k-essence 



\section{Lista de Figuras}

1.1 Gráfico original da primeira medida do parâmetro $H_{0}$, retirado de Hubble (1929a). . . . . . . . . .

1.2 Gráfico original da primeira medida da aceleração do Universo retirado de Riess et al. (1998). . . . . . . . . . . . . . 36

1.3 Gráfico retirado de Amanullah et al. (2010), mostrando os parâmetros de densidade obtidos por vários métodos observacionais diferentes. . . . . . . 41

1.4 Evolução das densidades energéticas do fluido cosmológico em função do

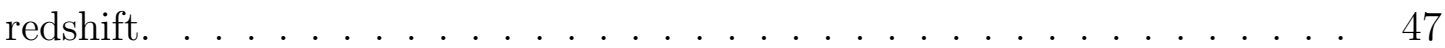

1.5 Evolução dos parâmetros de densidade do vácuo e da matéria no modelo

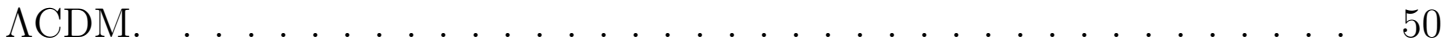

1.6 Gráfico sobre a tensão das medidas em $H_{0}$, retirado de Lima e Santos (2019). 53

2.1 Evolução das densidades energéticas do fluido cosmológico para o modelo $\Lambda(t) \ldots \ldots \ldots \ldots \ldots \ldots \ldots \ldots \ldots \ldots \ldots \ldots \ldots \ldots \ldots$

2.2 Gráficos dos parâmetros de densidade do vácuo e da matéria para os modelos $\Lambda \mathrm{CDM}$ e $\Lambda(t) \ldots \ldots \ldots \ldots \ldots \ldots$

4.1 Gráfico da evolução das densidades energéticas do fluido cosmológico para a descrição de campo escalar canônico do modelo $\Lambda(t) \ldots$. . . . . . . . . . 79

4.2 Gráfico da evolução das densidades energéticas do fluido cosmológico para a descrição de k-essência do modelo $\Lambda(t) \ldots$. . . . . . . . . . . . 



\section{Sumário}

Notações e Convenções . . . . . . . . . . . . . . . . . . . . . . . . . . . . 19

Introdução . . . . . . . . . . . . . . . . . . . . . . 21

1. Modelo $\Lambda C D M \ldots \ldots \ldots \ldots \ldots \ldots \ldots$

1.1 Equações de Einstein . . . . . . . . . . . . . . . . . . . . . . . . 27

1.1.1 Conservação de Energia para um Fluido Perfeito . . . . . . . . . . . 30

1.2 Equações de Friedmann . . . . . . . . . . . . . . . . . . . . . 32

1.2.1 Universo de Sitter . . . . . . . . . . . . . . . . . 33

1.3 Resultados Observacionais . . . . . . . . . . . . . . . . . 34

1.3.1 Expansão do Universo . . . . . . . . . . . . . . 35

1.3.2 Radiação Cósmica de Fundo . . . . . . . . . . . . 37

1.3.3 Matéria Escura . . . . . . . . . . . . . . 38

1.3.4 Medidas dos Parâmetros Observacionais . . . . . . . . . . 39

1.4 Inflação . . . . . . . . . . . . . . . . . . . . . . 42 42

1.4.1 Inflação Adiabática . . . . . . . . . . . . . . . 42

1.5 Dinâmica do Modelo . . . . . . . . . . . . . . . . 45

1.5.1 Evolução das Componentes Energéticas . . . . . . . . . . . 45

1.5.2 Evolução do Fator de Escala . . . . . . . . . . . . . 47

1.5.3 Idade do Universo . . . . . . . . . . . . . . . . . . 48

1.6 Problemas do Modelo $\Lambda \mathrm{CDM} \ldots \ldots \ldots$. . . . . . . . . . . . . . .

1.6.1 Problema da Coincidência . . . . . . . . . . . . 49

1.6.2 Problema da Constante Cosmológica . . . . . . . . . . . . . 5 50

1.6.3 Problemas com o Cenário Inflacionário . . . . . . . . . . . 52 
2. Modelos com Decaimento do Vácuo - $\Lambda(t) \ldots \ldots$. . . . . . . . . . 55

2.1 Conservação de Energia nos Modelos com $\Lambda(\mathrm{t})$. . . . . . . . . . . . 55

2.2 Lei de Decaimento do Vácuo . . . . . . . . . . . . . . . 56

2.3 Dinâmica do Modelo $\Lambda(\mathrm{t}) \quad \ldots \ldots \ldots$. . . . . . . . . . . . 59

2.3.1 Universo Primordial . . . . . . . . . . . . . . . . . 6 60

2.3.2 Universo Atual . . . . . . . . . . . . . . . 62

2.4 Possível Solução de Alguns Problemas do Modelo Padrão . . . . . . . . . . 64

2.4.1 Problema da Coincidência Cosmológica . . . . . . . . . . . 64

2.4.2 Problema da Constante Cosmológica . . . . . . . . . . . 65

3. Modelos com Campos Escalares: Campo Canônico e K-Essência . . . . . . . . 67

3.1 Dinâmica Cosmológica para o Campo Escalar Canônico . . . . . . . . . 68

3.2 O Caso Geral: Formulação Lagrangiana de Horndeski . . . . . . . . . . . . 69

3.2 .1 Quintessência ......................... 71

$3.2 .2 \quad$ K-Essência . . . . . . . . . . . . . . . . . . 71

4. K-Essência e Decaimento do Vácuo . . . . . . . . . . . . . . . . 75

4.1 Campo Escalar Canônico como Decaimento do Vácuo . . . . . . . . . . . 75

4.1.1 Interpretação do Campo escalar como Decaimento do Vácuo . . . . 75

4.1.2 Dinâmica Cosmológica para o Campo Escalar Canônico . . . . . . . 76

4.1.3 Campo escalar e o Universo Primordial em Cosmologias com Decaimento do Vácuo . . . . . . . . . . . . . . . . . . . . . 77

4.2 K-Essência como Decaimento do Vácuo . . . . . . . . . . . . . . . . 79

4.2.1 K-Essência como um Fluido Perfeito . . . . . . . . . . . 80

4.2.2 Dinâmica Cosmológica para a K-Essência . . . . . . . . . . . . 81

4.2.3 Universo Primordial na Descrição de Campo Escalar . . . . . . . . 82

5. Conclusões e Perspectivas . . . . . . . . . . . . . . . . 85

Apêndice

A. Tensor Energia-Momento do Vácuo . . . . . . . . . . . . . . . . . . . . . . 89 
B. Redshift, Distância de Luminosidade e Horizontes . . . . . . . . . . . . . . . . . 93

B.1 Redshift ................................. 93

B.2 Distância de Luminosidade . . . . . . . . . . . . . . . . . . . . . . 95

B.3 Horizontes . . . . . . . . . . . . . . . . . . . . . . . . . . . . . . . . 99

B.3.1 Horizonte de Eventos . . . . . . . . . . . . . . . . . . . . 99

B.3.2 Horizonte de Partículas . . . . . . . . . . . . . . . . . . . . 101

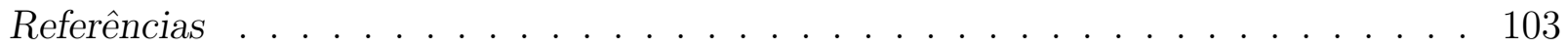





\section{Notações e Convenções}

- Empregaremos a métrica com a assinatura (+ - - -);

- Índices gregos variam de 0 a 3. Índices latinos variam de 1 a 3;

- Seguiremos a convenção de Einstein, onde índices superiores e inferiores repetidos indicam soma: $a^{\mu} a_{\mu} \equiv \sum_{i=0}^{3} a^{i} a_{i}$;

- No contexto de relatividade geral, utilizaremos a seguinte notação para a derivada parcial: $\frac{\partial f}{\partial x^{\alpha}} \equiv f_{, \alpha}$;

- Denotaremos a derivada covariante por: $A_{; \beta}^{\alpha} \equiv A_{, \beta}^{\alpha}+\Gamma_{\beta \lambda}^{\alpha} A^{\lambda}$;

- Ao longo do trabalho, utilizaremos unidades naturais onde $\hbar=c=k_{b}=1$. No caso de outras unidades serem empregadas, será dito explicitamente no texto;

- Nas unidades naturais, todas as dimensões podem ser escritas em termos da energia: $[$ energia $]=[$ massa $]=[$ temperatura $]=[\text { comprimento }]^{-1}=[\text { tempo }]^{-1}$. Utilizaremos o elétron-volt (eV) como unidade fundamental;

- A unidade de distância adotada será o megaparsec: $1 M p c=3,26 \times 10^{6}$ anos $-l u z=$ $3,09 \times 10^{22} \mathrm{~m}$. 


\section{Introdução}

A curiosidade sobre a natureza do Universo que nos cerca acompanha a humanidade desde seus primórdios. Nas sociedades primitivas, foram propostos modelos cosmogônicos explicando o surgimento do Cosmos através de mitos e lendas. Nesta introdução, faremos uma breve contextualização histórica aos modelos cosmológicos baseados na teoria da Relatividade Geral (RG), seguida por uma motivação dos problemas investigados no corpo da presente dissertação.

Na ciência, podemos nos referir ao sistema geocêntrico de Ptolomeu como o primeiro modelo cosmológico proposto. Este propunha uma descrição do movimento do Sol, da Lua, dos planetas (corpos errantes) e das estrelas distantes (fixadas na esfera celeste) em torno da Terra, estando esta, posicionada no centro do Universo. Este modelo foi sucedido pelo modelo Heliocêntrico, proposto inicialmente por Copérnico e aprimorado pelos trabalhos de Tycho Brahe e Johannes Kepler. Entretanto embora este modelo retirasse a Terra do centro do Universo, ele não modificava o plano de fundo em que este estava inserido e não surgia de nenhuma teoria física básica.

Naturalmente, esse último passo só foi possível após o desenvolvimento da Teoria de Gravitação Universal por, Isaac Newton. Sua teoria é capaz de deduzir, de primeiros princípios, as três leis do movimento planetário de Kepler e nos possibilitou perceber que as leis que governam os céus eram as mesmas que governavam o movimento dos "graves" (corpos massivos) nas proximidades da Terra. É importante ressaltar que Newton não modificou o modelo copernicano, apenas forneceu uma base teórica mais sólida para ele.

Apesar disso, a teoria Newtoniana possuía uma questão inquietante, pois não explicava de forma alguma como se dava a atração gravitacional, apenas mensurava seu efeito, dado 
que de acordo com sua teoria, a gravitação era uma força de ação à distância e instantânea. Inicialmente, esse era um problema notadamente filosófico, com origem no fato de ser contra intuitivo pensar em uma força atuando dessa forma. À medida que nossos conhecimentos na física avançaram, no entanto, esta questão se revelou fundamental, uma vez que indicava um conflito entre a Teoria de Mecânica Clássica com a Teoria do Eletromagnetismo de Maxwell; se revelando incompatível com o desenvolvimento da Teoria da Relatividade Especial (Einstein, 1905), que postulava que nenhuma informação poderia ser transmitida com velocidade superior à da luz (finita). Devido a esses problemas, Henri Poincaré, sugeriu que uma teoria capaz de unificar relatividade com gravitação necessitaria prever a existência de ondas gravitacionais (Poincaré, 1908).

Finalmente, em 1915 o próprio Einstein desenvolve a Teoria da Relatividade Geral (Einstein, 1915), com isso resolvendo os dois problemas que a teoria da gravitação Newtoniana possuía. Agora, a gravidade passa a ser interpretada como uma deformação do espaço-tempo, sendo esta propagada por meio de ondas gravitacionais que se deslocam na velocidade da luz (Carroll, 2004). Esse desenvolvimento permite o surgimento do primeiro modelo da Cosmologia moderna: o de universo estático de Einstein (Einstein, 1917), no qual a força de gravitação é contrabalanceada pela constante cosmológica. Nesse momento, o cenário cosmológico ainda é muito parecido com o Newtoniano, já que à época, ainda se imaginava que o Universo era composto apenas pela nossa Galáxia (Nemiroff e Bonnell 1999). No mesmo ano, de Sitter demonstra que em um universo vazio, em que apenas a constante cosmológica estivesse presente, seria possível se observar uma expansão acelerada e ininterrupta (de Sitter, 1917); ideia esta que, futuramente, seria utilizada para descrever a dinâmica do cenário inflacionário. Mais tarde este primeiro modelo é demonstrado ser instável e a ideia da constante cosmológica foi descartada por Einstein (tendo sido considerada por ele, seu maior erro (Gamow, 1956). Ali já estava presente a ideia principal e todo o artifício matemático que permitiria o desenvolvimento do modelo padrão cosmológico que conhecemos hoje.

Nos anos que se seguiram, os principais avanços para os modelos cosmológicos do ponto de vista observacional, foram as descobertas de que o Universo estava se expandindo (Hubble, 1929a) e que a nebulosa de Andrômeda (M31) estava fora da nossa Galáxia (Hubble, 1929b). Isso nos revelou que, além de não ser estático, o Universo é muito maior do que imaginávamos, modificando substancialmente o cenário cosmológico em que acreditávamos. Já 
do ponto de vista teórico, Friedmann obteve as soluções das equações de Einstein para um universo homogêneo, isotrópico e não estático (Friedmann, 1922, 1924); ao passo que, Georges Lemaître, de maneira independente, propõe a "Teoria do Big Bang", mostrando que a constante cosmológica poderia justificar a expansão de um Universo repleto de matéria (Lemaître, 1931). Posteriormente, Howard Robertson e Arthur Walker, demostraram que tais modelos eram as únicas soluções possíveis impostas as condições de um homogeneidade e isotropia (Robertson, 1935; Walker, 1935). Por esse motivo, a métrica, que é solução das equações de Einstein nesse caso, é conhecida como métrica de Friedmann-LemaîtreRobertson-Walker (FLRW).

Apesar de todo esse avanço, a cosmologia ainda não poderia ser considerada uma teoria física, pois não existiam observações, além da expansão do Universo, capazes de descartar (ou corroborar) os modelos teóricos sugeridos. Tal condição só foi alterada após a descoberta da Radiação Cósmica de Fundo (CMB, em inglês) (Penzias e Wilson, 1965), que havia sido proposta como evidência observacional da Teoria do Big-Bang Alpher et al. 1948).

A radiação cósmica pode ser imaginada como um banho de fótons, todos à mesma temperatura, que permeia o Universo. Ela é uma relíquia, um fóssil, da época em que os elétrons se fundiram com prótons e formaram os primeiros átomos de Hidrogênio: a época da Recombinação. A radiação é consideravelmente isotrópica, possuindo a temperatura média de $T=2,73 K$, com anisotropias de $\Delta T \approx 10^{-5} K$ (Mukhanov, 2005), sendo detectada em todas as direções da esfera celeste. Entretanto, essa isotropia se revelou um problema, já que o modelo proposto à época era incapaz de explicar como regiões sem conexão causal poderiam estar em equilíbrio térmico. Para explicar essa observação (conhecido como problema dos horizontes), foi proposto o chamado cenário inflacionário (Guth, 1981): um período no início do universo em que as escalas cosmológicas cresceram exponencialmente. Atualmente, esse cenário também explica o surgimento das sementes que originaram as galáxias e aglomerados (Liddle e Lyth, 2000); os componentes principais da estrutura de larga escala no Universo. Entretanto, a Inflação é adicionada ao modelo padrão apenas pela necessidade de explicar essa termalização do universo. De fato, ainda não sabemos a escala temporal em que ela ocorre e nem qual é a sua origem física 1 .

\footnotetext{
${ }^{1}$ Embora não possamos afirmar com certeza quando a inflação primordial aconteceu, sabemos que ela deve acontecer poucos segundos após o Big-Bang, $\delta t \approx 10^{-35} \mathrm{~s}$, com uma temperatura $T \approx 10^{14} \mathrm{GeV}$.
} 
Inicialmente, acreditava-se que uma expansão acelerada do universo havia ocorrido somente na sua fase inicial. Essa ideia revelou-se equivocada após observações de Supernovas do tipo Ia mostrarem que a expansão do universo acontecia de forma acelerada (Riess et al. 1998). Esse resultado foi surpreendente, uma vez que à época imaginava-se que essa expansão deveria ocorrer de maneira desacelerada, devido à natureza atrativa da interação gravitacional. Para explicar essa observação, era necessária a existência de uma densidade de energia com pressão negativa denominada Energia Escura, cuja melhor candidata era a constante cosmológica $(\Lambda)$, proposta originalmente por Einstein para manter o universo estático. Medidas atuais dos parâmetros de densidade nos apontam que a $\Lambda$ corresponde $70 \%$ da energia do Universo atual (Amanullah et al., 2010, Aghanim et al., 2018). Assim, temos todos os ingredientes presentes no modelo padrão aceito da cosmologia contemporânea, o $\Lambda$ CDM. Embora a energia escura seja comumente associada à densidade de energia do vácuo, ainda não sabemos de fato o que é essa energia, ou mesmo qual a sua origem. Existe uma vastidão de modelos que buscam solucionar esse problema, incluindo teorias de gravitação alternativas à Relatividade geral.

Nessa dissertação, iremos nos concentrar numa classe de modelos com decaimento do vácuo que buscam solucionar alguns dos problemas existentes no modelo padrão, sugerindo o vácuo como candidato à energia escura e um Universo que se origina, naturalmente, em uma fase inflacionária. Em seguida, iremos propor, na parte original do trabalho, uma maneira de descrever esse decaimento com um campo escalar, por meio da k-essência.

O presente trabalho seguirá a seguinte estrutura: no primeiro capítulo faremos uma discussão aprofundada sobre modelo $\Lambda \mathrm{CDM}$, apresentando sua descrição teórica e as principais evidências observacionais que o sustentam. Faremos uma síntese sobre sua dinâmica, mostrando a evolução temporal das componentes energéticas do fluido cosmológico. Em seguida, discutiremos alguns dos seus principais problemas, com enfoque especial nos problemas da constante cosmológica e da coincidência cósmica.

No segundo capítulo, discutiremos modelos com decaimento do vácuo. Apresentaremos uma breve revisão histórica da classe de modelos estudada e, em seguida, suas consequências para descrição da dinâmica do Universo. Nessa classe de modelos, o Universo parte de uma fase (quase) de Sitter primordial e evolui naturalmente para a fase da radiação, sendo livre do chamado "problema de saída graciosa". Sua evolução segue gradativamente para a fase da matéria e, finalmente, retorna a uma fase de expansão ace- 
lerada do tipo de Sitter. Mostraremos, ainda, como o modelo se propõe a resolver os dois problemas citados no parágrafo anterior.

No terceiro capítulo, discutiremos descrições de campo escalar para o estágio inflacionário. Partindo da ação de Einstein-Hilbert, mostraremos como podemos obter, por meio de uma forma lagrangeana, as equações de Friedmann para o campo canônico. Em seguida, utilizaremos a lagrangeana de Horndeski (Horndeski, 1974) para obter uma descrição de campo escalar mais geral, chamada k-essência, e as equações dinâmicas para essa descrição.

No quarto capítulo, apresentaremos a parte original do trabalho: uma descrição de campo escalar para o decaimento do vácuo utilizando a k-essência. Finalmente, no quinto capítulo, apresentaremos nossas conclusões e perspectivas. 
Capítulo 1

\section{Modelo $\Lambda \mathrm{CDM}$}

\subsection{Equações de Einstein}

Nesse capítulo, estudaremos o modelo cosmológico padrão, também chamado de modelo de concordância cósmica $(\Lambda \mathrm{CDM})$. A abordagem feita aqui é inspirada no Kolb e Turner (1990) e em Weinberg (2008a). O primeiro passo é resolver as equações de Einstein, portanto, precisamos determinar a métrica a ser utilizada. A nível geométrico, o modelo pode ser descrito pela métrica FLRW:

$$
g_{\mu \nu}=\left(\begin{array}{cccc}
1 & 0 & 0 & 0 \\
0 & -\frac{a(t)^{2}}{1-k r^{2}} & 0 & 0 \\
0 & 0 & -a^{2}(t) r^{2} & 0 \\
0 & 0 & 0 & -a^{2}(t) r^{2} \operatorname{sen}^{2} \theta
\end{array}\right) .
$$

em que $a(t)$ é o fator de escala e está relacionado com o raio do universo, $r^{2}=x^{2}+y^{2}+z^{2}$ representa a distância espacial física e $k$ é o parâmetro de curvatura espacial, que pode assumir três valores:

$$
k=\left\{\begin{array}{lll}
-1 & \rightarrow & \text { universo curvo hiperbólico. } \\
0 & \rightarrow & \text { universo plano. } \\
1 & \rightarrow & \text { universo curvo fechado. }
\end{array}\right.
$$

Podemos obter a versão contravariante da métrica por meio da relação $g^{\mu \beta} g_{\beta \nu}=\delta_{\nu}^{\mu}$. Note que apenas os termos diagonais são diferentes de zero e que o fator de escala depende exclusivamente do tempo. Isso significa que estamos modelando o Universo como homogêneo e isotrópico, condição que, de acordo com as observações, é respeitada para 
escalas $\approx 100 \mathrm{Mpc}$, sendo esta, portanto, a escala de validade para o modelo cosmológico não-perturbado. É possível mostrar que, a imposição de homogeneidade e isotropia implicam ser a métrica FLRW a única possível (Robertson, 1935; Walker, 1935).

Na relatividade geral, a métrica é o que nos permite calcular distâncias no espaço-tempo considerado. Fazemos isso através do intervalo: uma medida de distância na geometria curva e quadridimensional do espaço-tempo que depende da métrica espacial adotada. No caso da métrica FLRW, o quadrado do intervalo $\left(d s^{2}\right)$ assume a seguinte forma em coordenadas quasi-esféricas:

$$
\begin{aligned}
d s^{2} & =g_{\mu \nu} d x^{\mu} d x^{\nu} \\
& =d t^{2}-a^{2}(t)\left(\frac{d r^{2}}{1-k r^{2}}+r^{2} d \theta^{2}+r^{2} \operatorname{sen}^{2} \theta d \phi^{2}\right)
\end{aligned}
$$

Escolhendo um sistema de coordenadas comóveis, ou seja, movendo-se junto com o fluido cosmológico, pode-se inferir que a implementação do fator de escala impõe que as distâncias espaciais evoluam com o tempo. Em outras palavras, $a(t)$ é responsável por permitir a descrição dinâmica do Universo.

Agora que temos nossa métrica determinada, podemos modelar o Universo por meio das Equações de Einstein:

$$
G_{\mu \nu}=8 \pi G T_{\mu \nu}^{T}
$$

onde $G$ representa a constante de gravitação universal. O tensor energia-momento total acima está escrito na forma:

$$
T_{\mu \nu}^{T}=T_{\mu \nu}^{f}+T_{\mu \nu}^{\Lambda},
$$

onde $T_{\mu \nu}^{f}$ é o tensor energia-momento do fluido cosmológico e $T_{\mu \nu}^{\Lambda}=\frac{\Lambda}{8 \pi G} g_{\mu \nu}$ é o tensor energia momento associado com a constante cosmológica.

$G_{\mu \nu}$ é o tensor de Einstein, dado por:

$$
G_{\mu \nu}=R_{\mu \nu}-\frac{1}{2} g_{\mu \nu} R
$$

$R_{\mu \nu}$ e $R$ são, respectivamente, o tensor e o escalar de Ricci, determinados por: 


$$
\begin{gathered}
R_{\mu \nu}=R_{\mu \rho \nu}^{\rho}=\partial_{\rho} \Gamma_{\nu \mu}^{\rho}-\partial_{\nu} \Gamma_{\rho \mu}^{\rho}+\Gamma_{\rho \lambda}^{\rho} \Gamma_{\nu \mu}^{\lambda}-\Gamma_{\nu \lambda}^{\rho} \Gamma_{\rho \mu}^{\lambda}, \\
R=g^{\mu \nu} R_{\mu \nu} .
\end{gathered}
$$

Para determinarmos o lado esquerdo da equação (1.3), precisamos calcular, a partir da métrica (1.2), os símbolos de Christoffel (Conexões)

$$
\Gamma_{\mu \nu}^{\alpha}=\frac{1}{2} g^{\alpha \beta}\left(\partial_{\nu} g_{\beta \mu}+\partial_{\mu} g_{\nu \beta}-\partial_{\beta} g_{\mu \nu}\right)
$$

Utilizando a métrica (1.2), mostra-se que as únicas componentes não-nulas das conexões (1.8) são dadas por (Kolb e Turner, 1990):

$$
\begin{aligned}
& \Gamma_{i j}^{0}=a \dot{a} \delta_{i j}, \\
& \Gamma_{j 0}^{i}=\Gamma_{j 0}^{i}=\frac{\dot{a}}{a} \delta_{j}^{i}, \\
& \Gamma_{j k}^{i}=\frac{1}{2} g^{i l}\left(\partial_{k} g_{l j}+\partial_{j} g_{l k}-\partial_{l} g_{j k}\right) .
\end{aligned}
$$

Substituindo as equações acima em (1.6), verifica-se que as únicas componentes nãonulas do tensor de Ricci (diagonais) podem ser escritas como:

$$
\begin{aligned}
R_{00} & =-3 \frac{\ddot{a}}{a}, \\
R_{i j} & =-\left[\frac{\ddot{a}}{a}+2 \frac{\dot{a}^{2}}{a^{2}}+\frac{2 k}{a^{2}}\right] g_{i j} .
\end{aligned}
$$

Finalmente, o escalar de curvatura $R$, assume a seguinte forma:

$$
\begin{aligned}
R & =g^{\mu \nu} R_{\mu \nu}=g^{00} R_{00}+3 g^{i i} R_{i i} \\
& =-6\left(\frac{\ddot{a}}{a}+\frac{\dot{a}^{2}}{a^{2}}+\frac{k}{a^{2}}\right) .
\end{aligned}
$$

De posse das quantidades geométricas acima, o tensor de Einstein $G_{\mu \nu}$ fica determinado.

Precisamos ainda modelar o tensor de momento energia $T_{T}^{\mu \nu}$ que descreve as componentes energéticas do Universo. O tensor $T_{\Lambda}^{\mu \nu}=\frac{\Lambda}{8 \pi G} g^{\mu \nu}$ pode ser interpretado como o tensor energia-momento do vácuo (ver apêndice A), sendo sua energia e pressão dadas por: 


$$
\begin{aligned}
& p_{\Lambda}=T^{00}=\frac{\Lambda}{8 \pi G}, \\
& \rho_{\Lambda}=-T_{i}^{i}=-g_{i i} T^{i i}=-\frac{\Lambda}{8 \pi G} .
\end{aligned}
$$

O fluido cosmológico, descrito pelo tensor $T_{f}^{\mu \nu}$ é usualmente modelado por um fluido perfeito (Peebles, 1994),

$$
T_{f}^{\mu \nu}=\left(\rho_{f}+p_{f}\right) u^{\mu} u^{\nu}-p_{f} g^{\mu \nu},
$$

onde, em um referencial inercial, a 4-velocidade é $u_{\mu}=(1,0,0,0)$ e $\rho_{f}=\sum_{i} \rho_{i}$ é a soma da densidade de todas as componentes do fluido (vale, de maneira análoga, para a pressão). Em geral, as equações de estado das componentes materiais/energéticas são definidas por $p_{i}=\omega \rho_{i}$, sendo:

$$
\omega=\left\{\begin{array}{lll}
0 & \rightarrow & \text { poeira (matéria não-relativística). } \\
1 / 3 & \rightarrow & \text { radiação. } \\
1 & \rightarrow & \text { matéria rígida. }
\end{array}\right.
$$

A matéria rígida recebe esse nome pois nela a velocidade do som e da luz são a mesma; incluímos aqui sua definição pois, embora ela não faça parte da descrição do modelo padrão, nós a discutiremos posteriormente.

A princípio, poderíamos incluir também $\omega=-1$ para descrever o vácuo, mas, como dito anteriormente, deixamos esse papel para a constante cosmológica, de forma que, na nossa descrição, seria redundante acrescentá-lo aqui. Em alguns casos, no entanto, é conveniente incluir a densidade do vácuo como uma componente do fluido cosmológico. Quando isso for necessário, será dito explicitamente.

\subsubsection{Conservação de Energia para um Fluido Perfeito}

A relação para a conservação de energia do fluido cosmológico pode ser obtida de várias formas distintas e não constitui uma equação independente das equações de Einstein. De forma que, poderíamos obtê-la fazendo apenas uma manipulação algébrica das equações de Friedmann. Por generalidade, faremos a dedução partindo diretamente do tensor energiamomento do fluido cosmológico. Tomando a projeção da divergência do tensor de energiamomento na direção da 4-velocidade do elemento de volume do fluido, temos: 


$$
u_{\mu} T_{(f) ; \nu}^{\mu \nu}=0
$$

Substituindo 1.16 em 1.17, obtemos:

$$
u_{\mu}\left[\left(\rho_{(f)}+p_{(f)}\right)_{, \nu} u^{\mu} u^{\nu}+\left(\rho_{(f)}+p_{(f)}\right) u_{; \nu}^{\mu} u^{\nu}+\left(\rho_{(f)}+p_{(f)}\right) u^{\mu} u_{; \nu}^{\nu}-p_{(f), \nu} g^{\mu \nu}-p_{(f)} g_{; \nu}^{\mu \nu}\right]=0
$$

Sabemos que na geometria Riemmaniana, adotada na $R G$ e em outras teorias $(F(R)$, Brans-Dicke, etc), a derivada covariante da métrica é sempre nula $\left(g_{; \nu}^{\mu \nu}=0\right)$ e, pela normalização da 4 -velocidade $\left(u^{\mu} u_{\mu}=1\right)$, temos

$$
u_{\mu} u_{; \nu}^{\mu}=0
$$

Substituindo as expressões acima na equação para conservação da energia, obtemos

$$
\begin{aligned}
\left(\rho_{(f)}+p_{(f)}\right)_{, \nu} u^{\nu}+\left(\rho_{(f)}+p_{(f)}\right) u_{; \nu}^{\nu}-p_{(f), \nu} g^{\mu \nu} u_{\mu} & = \\
\rho_{(f), \nu} u^{\nu}+\left(\rho_{(f)}+p_{(f)}\right) u_{; \nu}^{\nu}-p_{(f), \nu} u^{\nu}+p_{(f), \nu} u^{\nu} & = \\
\rho_{(f), \nu} u^{\nu}+\left(\rho_{(f)}+p_{(f)}\right) & u_{; \nu}^{\nu}=0,
\end{aligned}
$$

sendo esta a expressão para a conservação de energia em uma métricas nas quais a 4aceleração é nula em RG. Agora, escolhendo coordenadas comóveis (que acompanham um elemento de volume do fluido), obtemos:

$$
\dot{\rho_{f}}+\left(\rho_{f}+p_{f}\right) \Theta=0
$$

onde $\Theta \equiv u_{; \nu}^{\nu}$ é a taxa de dissipação do fluido, que será definida pela escolha de uma métrica. Para a métrica de FLRW, podemos mostrar que $\Theta=3 \frac{\dot{a}}{a}$. Vemos que, nesse caso, a taxa de diluição de energia do fluido é dada pela taxa de expansão do universo. Substituindo esse resultado na equação (1.21) obtemos a seguinte relação para a conservação de energia:

$$
\dot{\rho_{f}}+3 H\left(\rho+p_{f}\right)=0
$$

onde $H \equiv \frac{\dot{a}}{a}$, é o chamado parâmetro de Hubble. É importante ressaltar que a equação de conservação de energia é válida, também, para cada fluido separadamente. 


\subsection{Equações de Friedmann}

Agora, temos todos os ingredientes necessários para resolver as equações de Einstein. A componente temporal nos dá uma equação, enquanto as três componentes espacias nos fornecem apenas mais uma equação. Assim, restam apenas duas equações independentes que governam a dinâmica do Universo, chamadas equações de Friedmann:

$$
\begin{aligned}
& 8 \pi G \rho_{f}+\Lambda=3 H^{2}+3 \frac{k}{a^{2}}, \\
& 8 \pi G p_{f}-\Lambda=-2 \dot{H}-3 H^{2}-\frac{k}{a^{2}},
\end{aligned}
$$

Dadas essas duas equações, podemos definir a densidade crítica $\rho_{c}$ como a densidade do fluido cosmológico tal que o Universo seja plano:

$$
\rho_{c} \equiv \frac{3 H^{2}}{8 \pi G}
$$

Esse parâmetro é importante pois nos permite definir o parâmetro de densidade $\Omega_{i}$ de cada componente energética do Universo, incluindo a densidade de energia do vácuo. Estes parâmetros podem ser determinados observacionalmente (Schneider, 2006) e descrevem a abundância relativa de cada componente:

$$
\begin{aligned}
\Omega_{i} & \equiv \frac{\rho_{i}}{\rho_{c}} \\
& \equiv \frac{8 \pi G}{3 H^{2}} \rho_{i} .
\end{aligned}
$$

Podemos, ainda, definir o parâmetro de densidade de curvatura $\Omega_{k}$ :

$$
\Omega_{k} \equiv-\frac{k}{a^{2} H^{2}}
$$

Escrevendo a equação 1.23 em termos dos parâmetros de densidade, obtemos

$$
\Omega_{T}+\Omega_{k}=1
$$

onde $\Omega_{T}=\sum_{i} \Omega_{i}$. Portanto, ao medirmos os parâmetros de densidade, podemos usá-los para retirar a informação sobre a curvatura do Universo: 


$$
\left\{\begin{array}{l}
\Omega_{T}>1\left(\Omega_{k}<0\right) \rightarrow k=1 . \\
\Omega_{T}=1\left(\Omega_{k}=0\right) \rightarrow k=0 . \\
\Omega_{T}<1\left(\Omega_{k}>0\right) \rightarrow k=-1 .
\end{array}\right.
$$

Conforme observado na Introdução, vários modelos cosmológicos foram propostos a partir das soluções das equações de Friedmann. Embora tais modelos possuam valor histórico e didático, a maioria foi descartada como uma possível descrição do Universo observado e, por esse motivo, não serão discutidos nesse texto.

Entretanto o universo de Sitter, em especial, proporciona uma dinâmica para o cenário inflacionário bastante intuitiva, descrevendo os estágios iniciais do Universo para a classe de modelos com decaimento do vácuo que será discutida mais adiante. A seguir, apresentaremos brevemente os principais resultados deste modelo.

\subsubsection{Universo de Sitter}

O universo de Sitter é modelado como um universo composto exclusivamente por vácuo, de forma que $\rho_{f}=0$ e $\Lambda=$ constante. Sob tais hipóteses, as equações de Friedmann 1.23 , 1.24) assumem as formas:

$$
\begin{aligned}
& \Lambda=3 H^{2}, \\
& \Lambda=2 \dot{H}+3 H^{2} .
\end{aligned}
$$

Substituindo 1.29 em 1.30 , obtemos $\dot{H}=0$. Isso implica que, durante toda a evolução do Universo, o parâmetro de Hubble é constante e dado por:

$$
H_{I}=\sqrt{\frac{\Lambda}{3}}
$$

A equação diferencial citada no parágrafo acima pode ser escrita em termos do fator de escala $\mathrm{a}(\mathrm{t})$ :

$$
\begin{aligned}
& \frac{\ddot{a}}{a}-\frac{\dot{a}^{2}}{a^{2}}=0, \\
\Rightarrow & \frac{\ddot{a}}{a}=H^{2} .
\end{aligned}
$$


Podemos ver que esse modelo nos fornece um universo sempre acelerado $(\ddot{a}>0$ ), representando uma inflação eterna. Por esse motivo, os modelos do tipo de Sitter podem ser utilizados para descrever estágios inflacionários tanto no universo primordial quanto no universo tardio. A solução da equação 1.33 é dada por:

$$
a(t)=a_{I} e^{H_{I} t}
$$

onde $a_{I}$ é o valor inicial do fator de escala nesse universo.

A expansão durante o estágio inflacionário pode ser quantificada pelo número de e-folds $N(t)$ :

$$
N(t) \equiv \ln \left[\frac{a_{*}}{a(t)}\right]
$$

onde $a_{*}$ é o fator de escala no fim do regime inflacionário $(\ddot{a}=0)$. Esse número nos diz a que potência do número de Euler $a(t)$ foi elevado e nos dá uma ideia do quão intensa foi a inflação. Para que a Inflação resolva os problemas a que se propõe (ver seção 1.4), são necessários de 40 a 60 e-folds (Kamionkowski e Kovetz, 2016).

Outra característica importante desse modelo é que ele é livre de horizonte de eventos ou, equivalentemente, é um universo não-singular (ver apêndice B.3).

\subsection{Resultados Observacionais}

Antes de apresentar a discussão da dinâmica desse modelo discutiremos os motivos pelos quais esse é o modelo padrão da cosmologia. Para isso precisamos apresentar os principais resultados observacionais que indicaram a sua validade: a expansão do universo Hubble, 1929a), a detecção da radiação cósmica de fundo (Cosmic Microwave Background, CMB) (Penzias e Wilson, 1965) e a descoberta da aceleração da expansão do Universo (Riess et al., 1998; Perlmutter et al., 1999). Embora esses sejam ingredientes imprescindíveis para a descrição e compreensão do modelo vigente, existem outros resultados igualmente importantes como, por exemplo, a bariogênese primordial, as anisotropias da CMB (Smoot et al., 1992; Hinshaw et al., 2013) e as oscilações acústicas dos bárions (Eisenstein et al. 2005) que não serão discutidos nesse texto. 


\subsubsection{Expansão do Universo}

A primeira observação a indicar a expansão do Universo foi feita por Edwin Hubble em 1929. Ele utilizou dados Cefeidas, as quais podem ser usadas como velas padronizáveis no Universo (ver apêndice B.2), para medir a distância de galáxias até nós, bem como mediu seu redshift para calcular a velocidade peculiar com que essas se afastavam (ou se aproximavam) da Via Láctea (Hubble, 1929a). Seus resultados originais estão apresentados no gráfico 1.1

Sua observação mostrou que a maioria das galáxias estava, de fato, se afastando de nós e obteve uma relação linear entre a distância de uma galáxia até nós e sua velocidade de afastamento,

$$
v=H_{0} d
$$

Esta foi a primeira medida do parâmetro $H_{0}$, batizado por esse motivo de parâmetro de Hubble. Entretanto, devido ao baixo número de supernovas utilizado e a sua proximidade com a Galáxia, o resultado obtido para esse parâmetro de, $500 \mathrm{Km} . \mathrm{s}^{-1} \mathrm{Mpc}^{-1}$, estava muito longe do aceito atualmente.
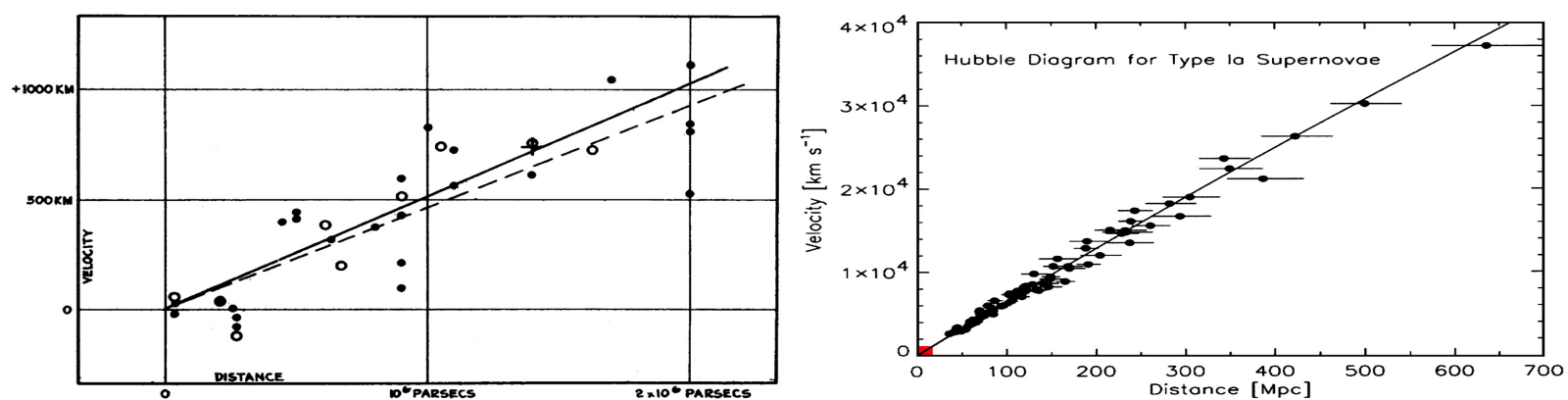

Figura 1.1: À esquerda, mostramos o gráfico original da primeira medida do parâmetro $H_{0}$, retirado de Hubble (1929a). À direita, temos o gráfico obtido em Kirshner (2004), uma observação mais atual que extende os resultados de Hubble. O quadrado vermelho no canto inferior esquerdo indica o espaço amostral utilizado por Hubble em sua medida original.

Ainda que esse resultado indicasse uma expansão do Universo, à época imaginava-se que essa expansão seria desacelerada devido à natureza atrativa da força de gravitação. Entretanto, em 1998, foi descoberto que essa expansão se dava de forma acelerada (Riess et al. 1998). Assim, se tornou necessário postular a existência de um mecanismo, denominado de Energia Escura (Dark Energy, DE), que justificasse essa aceleração. No gráfico 
1.2 mostramos os resultados de 2001, obtidos por dois grupos independentes, confirmando o estado acelerado do Universo.

Dado a observação da expansão acelerada do Universo, torna-se importante ver sob quais condições um fluido perfeito pode descrever essa dinâmica. Escrevendo as equações de Friedmann em termos do fator de escala, temos:

$$
\begin{aligned}
\frac{8 \pi G}{3} \rho_{T} & =\frac{\dot{a}^{2}}{a}, \\
8 \pi G \omega \rho_{T} & =-2 \frac{\ddot{a}}{a}-\frac{\dot{a}^{2}}{a},
\end{aligned}
$$

onde dividimos a primeira equação por 3 ; utilizamos $p=\omega \rho$ na segunda equação e não estamos incluindo o termo de constante cosmológica. Somando as equações acima, obtemos:

$$
\frac{\ddot{a}}{a}=-4 \pi G \rho\left(\omega+\frac{1}{3}\right)
$$

A condição para que o universo esteja acelerado é $\ddot{a}>0$. Como $a(t)>0 \forall t$, essa condição pode ser expressa por:

$$
\omega+\frac{1}{3}<0
$$

Ou seja, para que o universo seja acelerado, precisamos de uma componente energética cuja equação de estado tenha $\omega<-1 / 3$, sendo $\omega=-1$ a constante cosmológica.

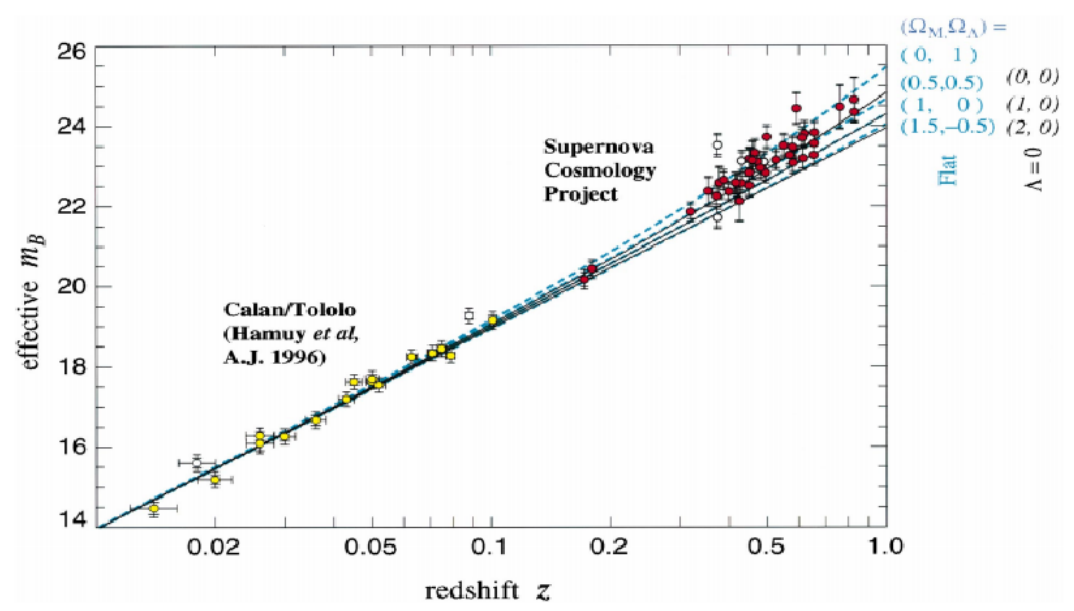

Figura 1.2: Gráfico retirado de Riess et al. (1998), que demonstrou a expansão acelerado do Universo.

A partir do descobrimento da expansão acelerada do Universo, projetos como o Dark Energy Task Force, foram criados para estudar a componente responsável por essa ace- 
leração e descobrir quem é a melhor candidata a D.E. Na verdade, a natureza da Energia Escura e sua possível interação com as outras componentes é uma grande questão em aberto na física atual.

\subsubsection{Radiação Cósmica de Fundo}

Devido à importância desse resultado, faremos uma descrição qualitativa do processo de formação desses fótons que compõe a CMB. Uma análise quantitativa e mais aprofundada pode ser encontrada nas referências: Kolb e Turner (1990), Dodelson (2003), e Weinberg (2008a).

A CMB é composta por fótons de baixa energia $\left(E \approx k_{B} t\right)$ de origem primordial, que permeiam todo o Universo. Ela foi originalmente proposta como evidência observacional do Big Bang por Alpher e Herman (1948), mas nessa época não havia o interesse (ou a tecnologia) necessária para fazer a sua detecção. Assim, ela foi esquecida pela maior parte da comunidade científica até a década de 1960, ressurgindo pelos trabalhos de Dicke et al. (1965) e Sunyaev e Zeldovich (1970), sendo observada, acidentalmente, pela primeira vez por Penzias e Wilson (1965). Esta detecção é considerada por muitos cosmólogos como o início da cosmologia observacional.

Para começar, precisamos estudar o Universo Primordial. Sabendo que o Universo está se expandindo e aceitando que as equações de Friedmann modelam bem essa expansão, pode-se mostrar que no início do Universo, $t \approx 0$, o fator de escala (ou raio do Universo) era muito pequeno, $a(t) \approx 0$ e, portanto, a densidade de energia era muito grande.

Nessa época, supõe-se que a matéria estivesse sujeita a temperaturas extremas (da ordem de $10^{12} \mathrm{~K}$ ); altas o suficiente para que os elétrons livres não conseguissem se ligar aos prótons para formar Hidrogênio e para que a matéria se mantivesse em equilíbrio térmico com os fótons. Assim, temos um ambiente que é opaco para os fótons, uma vez que eles estão constantemente colidindo com essa "sopa" de matéria relativística.

Entretanto, à medida que o Universo se expande, essa densidade de energia é diluída, o comprimento de onda dos fótons primordiais aumenta e o Universo esfria. Quando a temperatura cai o suficiente (algo entre $10^{5} \mathrm{~K}$ e $10^{3} \mathrm{~K}$ ), os elétrons passam a ser capazes de se ligar aos prótons e os fótons primordiais não possuem mais a energia necessária para romper a ligação. Essa é denominada a época da recombinação. Nesse momento, temos a formação dos primeiros átomos de hidrogênio e o Universo passa a ser "transparente" para 
os fótons, tornando possível a propagação desses fótons primordiais até hoje, formando a CMB. Eles continuaram a "esfriar" devido à expansão do Universo e, por esse motivo, hoje detectamos a radiação cósmica de fundo com a temperatura de aproximadamente $2,7 \mathrm{~K}$.

Outra característica marcante da CMB é que ela é aproximadamente isotrópica, com variações na sua temperatura da ordem de $10^{-5} \mathrm{~K}$. Ainda assim essas anisotropias são extremamente importantes, pois dão uma provável explicação para o processo de formação de grandes estruturas (Liddle e Lyth, 2000). Embora essa isotropia seja esperada, uma vez que é imaginado que os fótons primordiais tenham surgido simultaneamente, ela também é surpreendente, pois, se adotarmos o modelo $\Lambda \mathrm{CDM}$, apenas regiões no céu dentro do ângulo de $1^{\circ}$ estariam em contato causal no início do Universo. Isso significa que não existe uma explicação dentro do modelo padrão para que essas regiões estivessem à mesma temperatura. Esse problema foi resolvido graças ao cenário inflacionário, de forma que torna-se importante fazer uma breve apresentação desse cenário (ver seção 1.4).

\subsubsection{Matéria Escura}

Nessa seção, apresentarei uma discussão qualitativa sobre a importância da matéria escura para o modelo padrão da cosmologia. Provavelmente, a sua evidência mais conhecida (e que popularizou o termo matéria escura) são as curvas de rotação das galáxias (Rubin et al., 1980; Schneider, 2006). A teoria de gravitação Newtoniana prevê que a velocidade de rotação das estrelas em uma galáxia apresentaria dois tipos de comportamento: um para distâncias dentro do bojo da galáxia ${ }^{1}$ e outro para regiões fora desse bojo. No primeiro caso, a velocidade das estrelas deveria aumentar linearmente com a distância até o centro da galáxia, até atingir um valor máximo nas extremidades do bojo. A partir daí, é previsto que a velocidade das estrelas decaia proporcionalmente ao quadrado da distância até o centro da galáxia. No entanto, as observações mostram que, embora o primeiro comportamento seja confirmado, essa velocidade assume um valor aproximadamente constante para distâncias maiores que os limites do bojo galáctico. Isso só pode ser explicado se aceitarmos que a teoria de gravitação clássica está errada ou se postularmos a existência de uma massa extra distribuída na galáxia que não pode ser detectada visualmente. À essa matéria, deu-se o nome de matéria escura (Dark Matter, DM). Outro exemplo muito estudado é a chamada

\footnotetext{
${ }^{1} \mathrm{O}$ bojo é definido como um grupo esferoide de estrelas encontrado na região central da maioria das galáxias espirais e concentra uma densidade de massa maior do que o restante da galáxia.
} 
Bullet Cluster (Clowe et al., 2006), uma colisão entre dois aglomerados de galáxias em que medidas de raio-x mostram que o seu centro de massa está deslocado do centro de massa bariônica.

Na cosmologia, o papel mais importante da matéria escura é gerar as sementes primordiais que serão responsáveis pela formação das estruturas no Universo. Atualmente, acredita-se que esse processo ocorreu bottom-top (Peebles, 1982; Blumenthal et al., 1984), isto é, com a formação das estruturas menores (e.g galáxias) antes das estruturas maiores (e.g aglomerados de galáxias). Para que isso aconteça, é importante que no início da história cosmológica ela esteja desacoplada da radiação, recebendo o nome de matéria escura fria (Cold Dark Matter, CDM). A matéria escura, assim como a radiação e os bárions, é também diluída pela expansão. No entanto, por ser não-interagente, essa componente escura pode se aglomerar, gerando os poços de potencial que, posteriormente (após a recombinação), irão atrair os bárions. As oscilações acústicas do fluido de fóton- bárion, na última superfície de espalhamento, são capazes de quantificar a contribuição relativa da matéria não-relativística (CDM + bárions), sendo essa muito maior do que a contribuição bariônica (ver figura 1.3).

Existem outras evidências importantes para indicar a existência da matéria-escura, como a relação massa-luminosidade de aglomerados de galáxias obtida por meio de lentes gravitacionais (Wu et al., 1998) e as anisotropias da CMB. Ainda assim, sua existência e contribuição para o conteúdo cósmico é um tema de debate atual tanto na área de física de partículas quanto em teorias de gravitação alternativas.

Agora que vimos os principais resultados que dão suporte ao modelo $\Lambda$ CDM, discutiremos as medidas atribuídas aos parâmetros observacionais do modelo.

\subsubsection{Medidas dos Parâmetros Observacionais}

As observações mais recentes nos fornecem os seguintes parâmetros de densidade (Aghanim et al. 2018):

Curvatura: $\Omega_{k}=0,0007 \pm 0,0019$.

Matéria Escura: $\Omega_{d m 0} h^{2}=0,120 \pm 0,001$.

Matéria Bariônica: $\Omega_{m 0} h^{2}=0,0224 \pm 0,0001$. 
Aqui, definimos $h \equiv H_{0} /\left(100 K m \cdot s^{-1} \cdot M p c^{-1}\right)$. Esse parâmetro é utilizado pois existe uma discrepância nos valores observados de $H_{0}$ por diferentes métodos (ver subseção 1.6.4). Em geral, temos $0,66 \leq h \leq 0,73$.

O parâmetro de curvatura $\Omega_{k}$ pode indicar uma pequena curvatura positiva no Universo, no entanto o consenso da comunidade científica é de que esse resultado é consistente com a descrição de Universo plano, dado que por limitações inerentemente observacionais seria impossível obter um medida do parâmetro $\Omega_{k}$ identicamente nula.

Com o intuito de ser didático e de manter os resultados o mais simples possível, utilizaremos $h=0,7$ e $\Omega_{k}=0$ para determinar os parâmetros de densidade das componentes do Universo. Nesse caso, temos:

$$
\left\{\begin{array}{l}
\text { Energia Escura } \Omega_{\Lambda_{0}}=0,685 . \\
\text { Matéria Escura: } \Omega_{d m 0}=0,265 . \\
\text { Matéria Bariônica: } \Omega_{m 0}=0,050 .
\end{array}\right.
$$

As componentes restantes como radiação e neutrinos ocupam, atualmente, uma porcentagem tão pequena da energia total (da ordem de $0.03 \%$ ) que não são comparáveis com as outras. Por motivos didáticos, separei as componentes de matéria escura e bariônica nesse caso, mas daqui pra frente elas serão tratadas conjuntamente e denominadas pelo subíndice " $m$ ", já que elas são descritas por uma mesma equação de estado não-relativística $(p=0)$. Além disso, com exceção das sementes primordiais, ambas exercem o mesmo papel na dinâmica do Universo. Com essa distribuição das componentes, temos:

$$
\Omega_{\Lambda}+\Omega_{m}=0,685+0,315=1 .
$$

Os dados mencionados acima são obtidos por meio de observações da CMB. Existem vários formas de se obter os parâmetros de densidade, sendo que o modelo $\Lambda \mathrm{CDM}$ é o que melhor se ajusta a eles (ver gráfico 1.3). Por esse motivo, o $\Lambda$ CDM também é conhecido como modelo de concordância cósmica.

Uma vez que as observações nos indicam um universo plano $\left(\Omega_{k}=0\right)$, daqui em diante, consideraremos apenas modelos cosmológicos com $k=0$. Não é objetivo desse trabalho mostrar como esses parâmetros podem ser obtidos observacionalmente. O leitor interessado pode encontrar uma breve discussão sobre como obtê-los por meio de observações de 


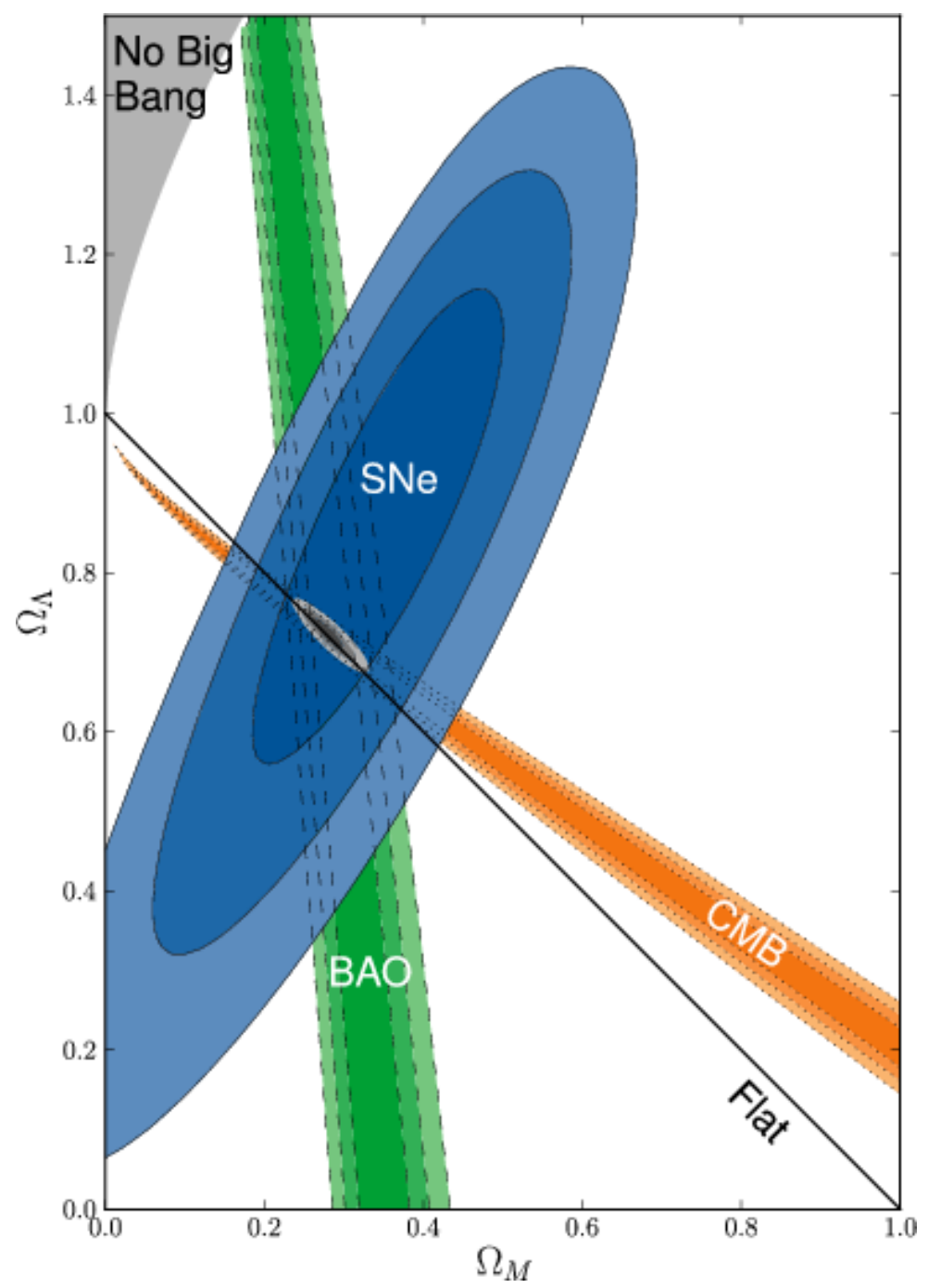

Figura 1.3: Gráfico retirado de Amanullah et al. (2010), mostrando os parâmetros de densidade obtidos por vários métodos observacionais diferentes. Repare que o modelo $\Lambda$ CDM plano com $\Omega_{\Lambda 0} \approx 0.7$ e $\Omega_{m 0} \approx 0.3$ é o escolhido na interseção dos métodos.

supernovas no apêndice B.2. Uma discussão mais aprofundada pode ser encontrada na referência Schneider (2006).

Além dos parâmetros de densidade, outro parâmetro observacional importante é o parâmetro de Hubble. Ele pode ser medido de várias maneiras, desde medidas diretas como a realizada por Hubble, como indiretamente, por meio do espectro de potências da CMB (Sunyaev e Zeldovich, 1980) e nos permite calcular, entre outras coisas, a idade do Universo. 


\subsection{Inflação}

A inflação é hoje parte imprescindível do modelo cosmológico padrão, uma vez que não só justifica a isotropia da CMB, como também fornece uma explicação para a origem das sementes primordiais (Hawking et al., 1982). Ainda assim, o processo constitui uma adição ad hoc ao modelo, já que, no caso $\Lambda \mathrm{CDM}$, a inflação não surge naturalmente das equações de Einstein.

O cenário inflacionário foi proposto pela primeira vez por Alan Guth em 1981 e tinha como principais objetivos solucionar dois problemas do modelo padrão (Guth, 1981):

- Problema de horizonte - regiões aparentemente desconectadas casualmente apresentam a mesma temperatura, medida através da radiação cósmica de fundo.

- Problema da planura do universo - para que o universo seja plano dentro do modelo padrão, precisamos de um ajuste fino do parâmetro de Hubble no início do universo.

Conforme visto na seção 1.2.1, durante a inflação o fator de escala cresce de forma exponencial $a(t) \propto e^{H_{I}(t)}$. Com esse grande aumento do fator de escala, as densidades volumétricas de todas as componentes do fluido caem para próximo de zero, levando o universo à um estado de baixa temperatura.

Nesse modelo de inflação, o mecanismo proposto para o reaquecimento do universo seriam colisões entre as "bolhas de verdadeiro vácuo". Quando essas bolhas se chocam, elas liberam energia, causando assim a termalização do universo. Entretanto, esse mecanismo não é eficiente (Hawking et al., 1982; Guth e Weinberg, 1983).

Dessa forma, embora o crescimento exponencial do fator de escala consiga resolver os dois problemas propostos, esse cenário se torna implausível, uma vez que não consegue explicar o reaquecimento do universo. Assim, novos cenários inflacionários foram propostos.

\subsubsection{Inflação Adiabática}

A diferença desse cenário, também conhecido como Novo Cenário Inflacionário, para o cenário proposto originalmente por A. Guth, é que o fluido cosmológico não irá decair para o verdadeiro vácuo por meio do tunelamento. O fluido, descrito por um campo escalar $\phi$, o inflaton, decai lentamente até um valor $\phi_{e}$. Esse processo se dá devido à existência de 
um potencial $V(\phi)$, que, durante essa fase, é aproximadamente plano. Essa é a chamada fase da inflação. Após esse valor, o campo oscila em torno do verdadeiro vácuo, decaindo nas outras componentes e reaquecendo o universo.

Esse cenário não leva em conta nenhuma dissipação do inflaton durante a inflação. Portanto, podemos escrever a equação para a conservação de energia do fluido da maneira usual:

$$
\dot{\rho_{T}}+3 H\left(p_{T}+\rho_{T}\right)=0 .
$$

Em se tratando do início do universo, estamos na era da radiação. Dessa forma temos $\rho_{T}=\rho_{r}+\rho_{\phi}$ e $p_{T}=p_{r}+p_{\phi}$. Neste ponto, apresentaremos apenas alguns resultados básicos referentes à inflação gerada por um campo escalar 2 . A densidade e pressão do campo do inflaton são dados por (Liddle e Lyth, 2000):

$$
\rho_{\phi}=\frac{\dot{\phi}^{2}}{2}+V(\phi), \quad p_{\phi}=\frac{\dot{\phi}^{2}}{2}-V(\phi)
$$

Substituindo as expressões acima nas equações (1.23) e (1.41), obtemos as equações que governam a dinâmica do campo escalar:

$$
\begin{aligned}
3 M_{P}^{2} H^{2} & =\frac{\dot{\phi}^{2}}{2}+V(\phi), \\
-2 M_{P}^{2} \dot{H}-3 M_{P}^{2} H^{2} & =\frac{\dot{\phi}^{2}}{2}-V(\phi) .
\end{aligned}
$$

Podemos obter também a equação para conservação de energia do campo escalar, substituindo as equações para a pressão e densidade do capo escalar 1.42 na equação (1.41):

$$
\ddot{\phi}+3 H \dot{\phi}+V^{\prime}(\phi)=0
$$

onde $V^{\prime} \equiv \frac{d V}{d \phi}$. Essa equação define a evolução temporal do campo escalar que descreve inflação. Pode-se utilizar teorias de perturbação nela para mostrar de onde surgem as anisotropias da CMB (Liddle e Lyth, 2000).

\footnotetext{
${ }^{2}$ Mais detalhes sobre o formalismo de campo escalar podem ser encontrados no capítulo 3
} 
Quando a energia cinética do campo é muito menor do que o potencial $\left(\frac{\dot{\phi}^{2}}{2}<<V(\phi)\right)$, temos $\rho_{\phi} \approx-p_{\phi}$, sendo essa fase conhecida como "rolamento lento". Por esse motivo o campo escalar pode ser utilizado para descrever o vácuo durante essa fase. Utilizando as equações de Friedmann e as aproximações de rolamento lento, temos:

$$
\begin{aligned}
H^{2} & \approx \frac{V(\phi)}{3 M_{P}^{2}}, \\
3 H \dot{\phi} & \approx V^{\prime}(\phi) .
\end{aligned}
$$

Definam-se os parâmetros de rolamento lento (Liddle e Lyth, 2000):

$$
\begin{aligned}
\epsilon & \equiv \frac{1}{2 k}\left(\frac{V^{\prime}}{V}\right)^{2}, \\
\eta & \equiv \frac{1}{k} \frac{V^{\prime \prime}}{V}
\end{aligned}
$$

onde $k=\frac{1}{M_{P}{ }^{2}}$ e $M_{P}=\frac{1}{\sqrt{8 \pi G}}$ é a massa de Planck reduzida.

O parâmetro $\epsilon$ é uma medida do quão rápido o rolamento do campo acontece, enquanto o parâmetro $\eta$ é uma medida da persistência (ou aceleração) desse processo. A condição para que ocorra rolamento lento é $\{\epsilon,|\eta|\}<<1$, de modo que, quando $\epsilon=1$ temos o fim da inflação.

Agora, substituindo as aproximações das equações de Friedmann na definição dos parâmetros de rolamento lento, podemos reescrevê-los em termos do parâmetro de Hubble:

$$
\begin{aligned}
\epsilon & =-\frac{\dot{H}}{H^{2}}, \\
\eta & =2 \epsilon-\frac{\dot{\epsilon}}{2 H \epsilon} .
\end{aligned}
$$

Esse cenário ainda exige uma fase de reaquecimento do universo depois da inflação. Esse processo de "pasteurização" parece pouco natural. Com isso em mente, foram propostos cenários alternativos como a k-inflação (Berera, 1995, Armendariz-Picon et al., 2001) e a inflação morna (Gron, 2016).

Agora que apresentamos os principais argumentos para sustentar o modelo $\Lambda$ CDM e os dados observacionais mais recentes, estudaremos a sua dinâmica. 


\subsection{Dinâmica do Modelo}

Em particular, estamos interessados em mostrar a evolução das densidades energéticas de cada componente do fluido cosmológico $\rho_{i}$, a evolução temporal do fator de escala $a(t)$ e a idade do Universo.

Conforme anteriormente, partiremos das equações de Friedmann para estudar a evolução do Universo. No entanto, para nossos propósitos, será útil escrevê-las em outro formato. Para isso substituiremos a $\operatorname{EoS} p=\omega \rho$ na equação (1.24) e somaremos as duas equações

$$
\dot{H}+\frac{3(1+\omega)}{2} H^{2}-\frac{(1+\omega)}{2} \Lambda=0 .
$$

Essa é a equação para a dinâmica do Universo e nos fornece a evolução temporal do parâmetro de Hubble (e alternativamente, do fator de escala). Entretanto, ela não nos indica, explicitamente, nenhuma informação sobre a evolução das componentes energéticas. Esse resultado pode ser obtido utilizando a equação de conservação de energia para o fluido cosmológico (1.22). Mas antes, mostraremos como ela está contida nas equações de Friedmann. Para isso basta tomar a derivada temporal da equação (1.23):

$$
8 \pi G \dot{\rho_{T}}=3 H\left(2 \frac{\ddot{a}}{a}-2 \frac{\dot{a}^{2}}{a^{2}}\right) .
$$

Note que o termo em parênteses na equação acima pode ser obtido multiplicando as equações 1.23 e 1.24 por -1 e somando as duas. Daí:

$$
\begin{gathered}
8 \pi G \dot{\rho_{f}}=24 \pi G H\left(-\rho_{f}-\Lambda-p_{f}+\Lambda\right), \\
=-24 \pi G H\left(\rho_{f}+p_{f}\right) . \\
\Rightarrow \dot{\rho}_{f}+3 H\left(\rho_{f}+p_{f}\right)=0 .
\end{gathered}
$$

Isso demonstra que a lei de conservação de energia está contida implicitamente nas equações de Friedmann. Esse resultado é apenas uma observação interessante sobre as equações e mostra que elas, de fato, contém todo o arcabouço necessário para a análise da dinâmica cosmológica.

\subsubsection{Evolução das Componentes Energéticas}

Para simplificar, faremos a suposição de que o fluido cosmológico é composto por apenas uma componente. Essa suposição é válida para modelar momentos da história cosmológica 
em que uma componente é dominante sobre as outras (e.g universo primordial $\rho_{r}>>$ $\left.\rho_{m}, \rho_{\Lambda}\right)$ ou quando não existe uma interação significativa entre as componentes, de forma que elas se conservem separadamente. E, embora uma análise de multifluidos forneça uma análise mais robusta, os resultados que vamos obter nos fornecem intuições interessantes sobre o comportamento das densidades energéticas.

Começaremos reescrevendo a equação de conservação de energia na forma a seguir,

$$
\frac{d}{d a}\left(\rho_{i} a^{3}\right)=-3 p_{i} a^{2}
$$

É fácil demonstrar que, ao efetuar a derivação acima, recuperamos a forma da equação 1.22. Substituindo a EoS na expressão acima e efetuando a integração de um tempo arbitrário $t$ até o tempo atual $t_{0}$, obtemos:

$$
\ln \left(\frac{\rho_{0 i} a_{0}^{3}}{\rho_{i} a^{3}}\right)=-3 \omega \ln (a)+c_{1},
$$

Com uma simples manipulação algébrica, podemos reescrever essa equação na seguinte forma:

$$
\rho_{i}=\rho_{0 i}\left(\frac{a_{0}}{a}\right)^{3(1+\omega)}
$$

Assim, para os valores de $\omega=-1,0,1 / 3,1$ (ver seção 1.1), temos:

$$
\rho_{i}= \begin{cases}\rho_{\Lambda 0}, & \text { vácuo. } \\ \rho_{m 0}\left(\frac{a_{0}}{a}\right)^{3}, & \text { matéria bariônica. } \\ \rho_{r 0}\left(\frac{a_{0}}{a}\right)^{4}, & \text { radiação. } \\ \rho_{s 0}\left(\frac{a_{0}}{a}\right)^{6}, & \text { matéria rígida. }\end{cases}
$$

Incluímos a matéria rígida nessa discussão pois ela será discutida mais à frente, no entanto, ela não é uma componente considerada no modelo padrão.

Desse resultado podemos retirar informações interessantes. A primeira delas é que, sob a suposição de que o vácuo não interaja com as outras componentes, sua densidade de energia permanece constante durante toda a história do Universo, sendo o seu valor igual a $\frac{\Lambda}{8 \pi G}$. Ou seja, seu valor é extremamente pequeno e só passa a ser relevante quando as outras componentes já foram muito diluídas (ver gráfico 1.4). Além disso, como o universo 


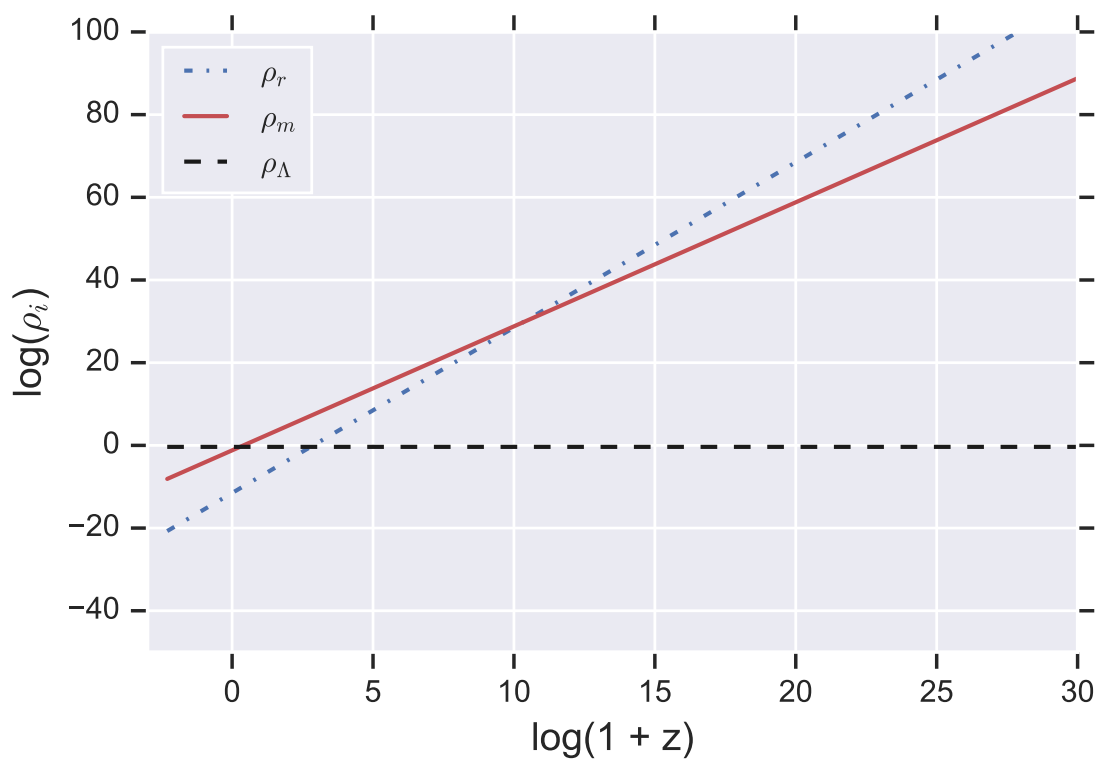

Figura 1.4: Gráfico das densidades energéticas do fluido cosmológico em função do redshift. Utilizamos a escala logaritmica devido à magnitude das grandezas, repare que $z$ grande indica passado, $z$ negativo o futuro e sendo $z=0$ o presente. Supondo $\Lambda=c t e$., vemos como cada componente tem uma fase dominante

seguindo a ordem: radiação, matéria, vácuo. Nesse gráfico, incluímos apenas as componentes que fazem parte do modelo padrão.

está em expansão, vemos que a radiação é diluída mais rapidamente do que a matéria, ou seja, mesmo que o Universo comece em uma era dominada pela radiação, eventualmente a matéria irá dominar e, em seguida, o vácuo, por menores que sejam suas densidades iniciais. Abaixo, mostramos o gráfico das densidades em função de $a(t)$.

Essa análise é suficiente para entender como as densidades energéticas evoluem com o tempo e ter uma ideia de quais são as fases pelas quais o Universo evolui. No entanto, para saber a relação explícita dessa evolução com o tempo, precisamos estudar a evolução do fator de escala.

\subsubsection{Evolução do Fator de Escala}

Para obter a evolução temporal do fator de escala, precisamos resolver a equação (1.52). Utilizando $\dot{H}=a H H^{\prime}$, onde linha denota derivação em relação a $a(t)$, podemos escrevê-la 
em sua forma integral e resolvê-la em termos do fator de escala:

$$
\begin{gathered}
\int_{H_{0}}^{H(t)} \frac{H d H}{\left(3 H^{2}-\Lambda\right)}=-\frac{(1+\omega)}{2} \int_{a_{0}}^{a(t)} \frac{d a}{a} \\
\frac{1}{6} \ln \left(\frac{\Lambda-3 H_{0}^{2}}{\Lambda-3 H^{2}}\right)=-\frac{(1+\omega)}{2} \ln \left(\frac{a_{0}}{a(t)}\right) \\
H(a)=\sqrt{\frac{\Lambda}{3}-\left(\frac{\Lambda}{3}-H_{0}^{2}\right)\left(\frac{a_{0}}{a(t)}\right)^{3(1+\omega)}}
\end{gathered}
$$

onde o subíndice "0" representa o valor de uma quantidade no tempo atual e descartamos a solução negativa pois, por construção, sabemos que o parâmetro de Hubble é estritamente positivo. No modelo padrão, o valor de $\Lambda$ é muito pequeno, portanto, vamos assumir $\Lambda=0$ para ilustrar as fases dominadas pelas componentes do fluido cosmológico. Assim, a equação $1.58 \mathrm{c}$ assume a forma

$$
\frac{\dot{a}}{a}=H_{0}\left(\frac{a_{0}}{a}\right)^{\frac{3}{2}(1+\omega)}
$$

cuja solução é dada por:

$$
a(t)=a_{0}\left[\frac{3(1+\omega)}{2} H_{0} t\right]^{\frac{2}{3(1+\omega)}},
$$

em que definimos $a(t=0) \equiv 0$.

Lembrando que, nesse caso o valor $\omega=-1$ não é permitido, pois não estamos incluindo o vácuo. Isso significa que essa descrição não permite um universo acelerado, como vimos na seção 1.3.1. Mesmo assim, esse é um resultado interessante, pois nos permite inferir como o fator de escala evolui nas fases dominadas pela matéria e radiação e será reproduzido quando apresentarmos os modelos com $\Lambda(t)$.

\subsubsection{Idade do Universo}

Com os valores de $\Omega_{i}$ e as equações para a evolução temporal de $\rho_{i}$, podemos calcular a idade do Universo. Para isso, iremos reescrever a equação 1.23 em termos dos parâmetros de densidade,

$$
\begin{aligned}
H & =H_{0} \Omega_{T}^{1 / 2} \\
& =H_{0}\left[\Omega_{\Lambda 0}+\Omega_{m 0}\left(\frac{a_{0}}{a}\right)^{3}+\Omega_{r 0}\left(\frac{a_{0}}{a}\right)^{4}\right]^{1 / 2}
\end{aligned}
$$


Agora, fazendo a mudança de variável $x=\frac{a}{a_{0}}$ e, utilizando $\frac{a}{a_{0}}=\frac{1}{1+z}$ onde "z"é o redshift (ver apêndice B.1), temos a expressão para a idade do universo em um redshift qualquer:

$$
t(z)=\frac{1}{H_{0}} \int_{0}^{\frac{1}{1+z}} \frac{d x}{x \sqrt{\Omega_{\Lambda 0}+\Omega_{m 0}\left(\frac{a_{0}}{a}\right)^{3}+\Omega_{r 0}\left(\frac{a_{0}}{a}\right)^{4}}} .
$$

A idade atual do Universo é obtida quando tomamos $z=0$ :

$$
t_{0}=\frac{1}{H_{0}} \int_{0}^{1} \frac{d x}{x \sqrt{\Omega_{\Lambda 0}+\Omega_{m 0} x^{-3}+\Omega_{r 0} x^{-4}}} .
$$

Para obtermos o valor da idade do Universo basta substituir os valores de $H_{0}$ e dos parâmetros de densidade e, então, efetuar a integração. Utilizando os valores informados na seção 1.3 .4 obtemos o resultado $t_{0} \approx 1.3 \times 10^{10}$ anos, ou seja, aproximadamente 13 bilhões de anos.

Tendo discutido o modelo $\Lambda \mathrm{CDM}$ e os motivos que sugerem sua validade, veremos a seguir quais são os problemas que o modelo possui e o porquê da necessidade de propormos modelos alternativos. Mas, com essa discussão, espero ter convencido o leitor de que o modelo é extremamente bem fundamentado e que, portanto, qualquer modelo alternativo deve reproduzir os resultados de $\Lambda$ CDM em algum limite.

\subsection{Problemas do Modelo $\Lambda$ CDM}

Nessa seção, apresentaremos alguns dos problemas do modelo padrão. Nos concentraremos principalmente em dois problemas: o da constante cosmológica (PCC) e o da coincidência (PC). Esses problemas serão discutidos novamente no capítulo sobre os modelos com $\Lambda(t)$, onde apresentaremos possíveis soluções para ambos os problemas.

Além dos problemas com o modelo $\Lambda \mathrm{CDM}$, existem questões em aberto com o cenário inflacionário. Faremos uma breve apresentação de alguns desses problemas uma vez que eles também serão retomados mais à frente.

\subsubsection{Problema da Coincidência}

Para entender esse problema, precisamos voltar nossa atenção à evolução dos parâmetros de densidade. Para isso, é conveniente escrevê-los em termos do redshift: 


$$
\Omega_{i}=\Omega_{i 0}\left[\Omega_{\Lambda 0}+\Omega_{m 0}(1+z)^{3}+\Omega_{r 0}(1+z)^{4}\right]^{-1}(1+z)^{-3(1+\omega)} .
$$

Podemos ver no gráfico 1.5 abaixo que, embora durante quase toda a história do Universo os parâmetros de densidade do fluido e do vácuo sejam incomparáveis, sendo $\Omega_{f}$ dominante durante o início do Universo e $\Omega_{\Lambda}$ dominante no futuro, atualmente as duas grandezas possuem a mesma escala de grandeza. Isso gera o questionamento do porque vivemos em uma época única na história cosmológica.

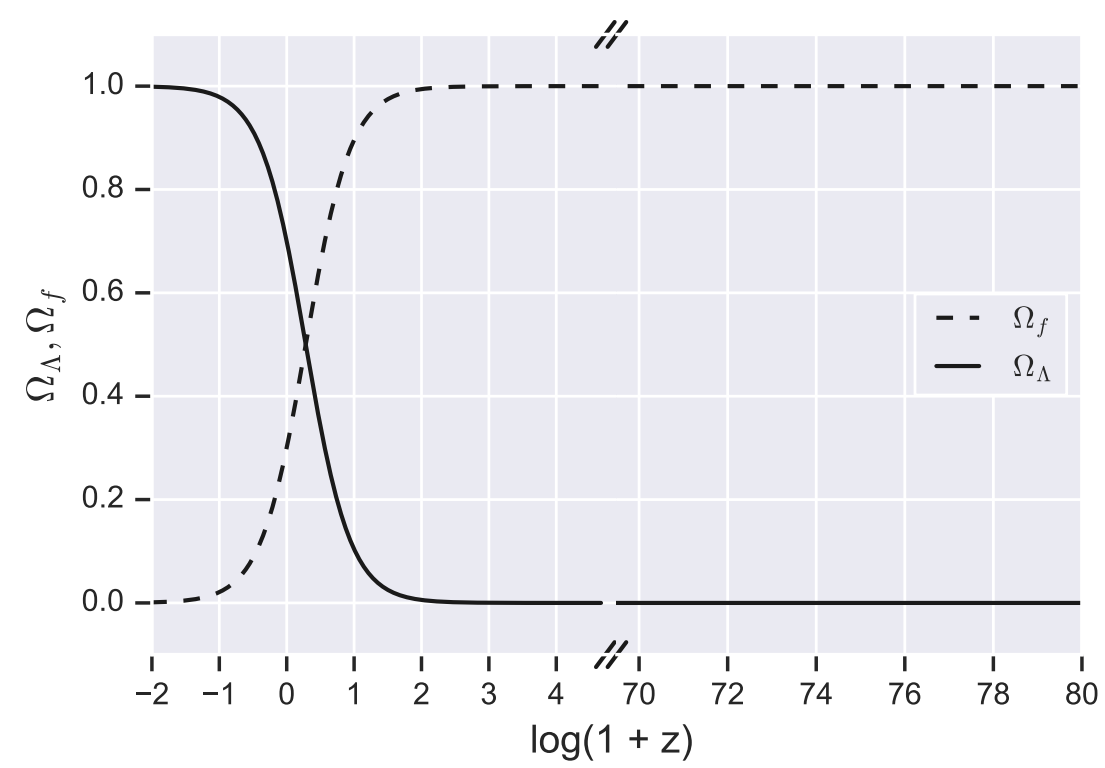

Figura 1.5: A linha tracejada representa o parâmetro de densidade do fluido cosmológico (matéria e radiação) e a linha contínua representa o parâmetro de densidade do vácuo. Utilizamos $\Omega_{\Lambda_{0}}=0.7 \mathrm{e}$ $\Omega_{m 0}=0.3$. Vemos que no início do Universo o fluido contém aproximadamente toda a energia disponível, mas próximo do tempo atual, ocorre uma inversão. De forma que, atualmente as duas densidades são comparáveis.

Naturalmente, esse não é um problema físico, uma vez que não existe nenhuma lei física que não permita essa coincidência, mas sim um problema filosófico, já que para que isso ocorra, as constantes físicas precisam assumir valores específicos entre uma infinidade de possibilidades.

\subsubsection{Problema da Constante Cosmológica}

Esse problema foi discutido por Stenven Weinberg em um artigo de revisão (Weinberg, 1989), onde ele não somente apresenta o problema como também expõe algumas possíveis soluções. 
De maneira simples, podemos enunciar esse problema como o desacordo entre o valor observacional medido para a densidade de energia do vácuo e sua estimativa teórica. Podemos determinar a densidade energética do vácuo atualmente $\rho_{\Lambda 0}$ através da expressão (1.26):

$$
\begin{aligned}
\rho_{\Lambda 0} & =\frac{3 H_{0}^{2}}{8 \pi G} \Omega_{\Lambda 0}, \\
& \approx 0.7 \times \rho_{c 0}, \approx 10^{-47} \mathrm{GeV}^{4} .
\end{aligned}
$$

Seu valor teórico pode ser previsto via teoria quântica de campos, calculando a energia de ponto zero de um campo quântico de massa $m$ fazendo sua renormalização com o cutoff em $\Lambda>>$ (Weinberg, 1989):

$$
\rho_{\Lambda 0} \approx \frac{\Lambda^{4}}{16 \pi^{2}}
$$

É importante salientar que nessa equação $\Lambda$ não é a constante cosmológica, apenas uma escala de energia. Assumindo a validade da relatividade geral até a escala de Planck, temos $\Lambda \approx \frac{1}{\sqrt{8 \pi G}}$. Substituindo em 1.66 obtemos,

$$
\rho_{\Lambda 0} \approx 10^{71} \mathrm{GeV}^{4}
$$

Comparando o valor observado com o valor teórico,

$$
\frac{\rho_{\text {teo }}}{\rho_{\text {obs }}} \approx 10^{118}
$$

observamos uma discrepância de 118 ordens de grandeza entre os dois valores, claramente um fracasso da teoria.

À primeira vista, alguém poderia pensar que esse problema pode ser evitado se interpretarmos $\Lambda$ como uma correção na teoria de gravitação Newtoniana ao invés da densidade de energia do vácuo. Nesse caso, teríamos duas componentes responsáveis pela aceleração do Universo e, para essa discussão, vamos denominar a soma de ambas por energia escura, de forma que

$$
\rho_{D E}=\rho_{V}+\Lambda
$$

onde $\rho_{V}$ é a densidade de ponto zero dos campos quânticos, dada por 1.66) e $\Lambda$ é a constante cosmológica geométrica. No entanto, nesse caso o problema toma uma forma 
parecida com o PC: uma vez que sabemos que atualmente o valor da densidade energética da DE é aproximadamente zero, seria surpreendente que $\Lambda$ tivesse um valor tal que, 13 bilhões de anos após o Big Bang (i.e. no momento em que estamos vivos) este cancelasse a contribuição dos campos quânticos. Ou seja, esse interpretação não é capaz de resolver o problema.

Temos ainda duas questões a serem levantadas no que diz respeito à interface entre o modelo $\Lambda$ CDM e o cenário inflacionário. A primeira delas é o fato de que o modelo padrão é incapaz de fornecer uma origem natural para a inflação, de forma que, atualmente, ela é um "enxerto" no modelo. A segunda questão é mais uma provocação do que de fato um problema: sabemos que o Universo hoje em dia está em expansão acelerada, se utilizarmos a definição fornecida na seção 1.1.1, essa seria uma fase inflacionária. Dessa forma será que existe alguma conexão entre o mecanismo que gera a inflação primordial e o que acelera o Universo atualmente?

Aproveitando o momento, seguiremos para a apresentação dos problemas da Inflação propriamente dita.

\subsubsection{Problemas com o Cenário Inflacionário}

Como vimos, é necessário que a inflação ocorra pouco após o Big Bang para que ela seja capaz de resolver os problemas dos horizontes e da planura universo. Entretanto, como não temos um mecanismo para justificar a sua origem, a escala de energia exata (ou tempo) em que ela se inicia ainda é desconhecido.

Vimos também que ela deve durar tempo suficiente para que o número de e-folds gerados seja (ao menos) maior do que 40, mas ela também não pode se alongar por muito tempo, pois nesse caso a radiação seria extremamente diluída nesse processo e o Universo seria frio demais para que ocorresse a bariogênese primordial. Sendo assim, existe um equilíbrio delicado no seu tempo de duração que deve ser respeitado, de forma que ela dure o suficiente para resolver os problemas, mas que o Universo saia dessa fase em condições de produzir os elementos que observamos atualmente. Esse problema é conhecido como "problema da saída graciosa" (graceful exit). 


\subsubsection{Tensão Supernova x CMB}

Um dos grandes problemas cosmológicos em aberto atualmente é a tensão na medida de $H_{0}$. Ela surge pois dois dos métodos mais confiáveis de se efetuar a medida, por meio de Supernovas do Tipo Ia (medida local), que fornece $H_{0}=73,8 \pm 2,4 \mathrm{~km} \cdot \mathrm{s}^{-1} \cdot \mathrm{Mpc}^{-1}$ (Riess et al., 2011) e através da CMB (medida global), $H_{0}=67,4 \pm 1,4 k m . s^{-1} \cdot M p c^{-1}$ (Aghanim et al. 2018), possuem uma discrepância acima dos seus erros (ver gráfico 1.6). Como o método por meio da CMB depende da suposição de um modelo cosmológico a priori, uma das possíveis explicações para a origem dessa tensão é que o modelo $\Lambda$ CDM não seja o correto, embora um modelo alternativo precise se aproximar dele, visto que todas as outras evidências observacionais apontam a sua validade.

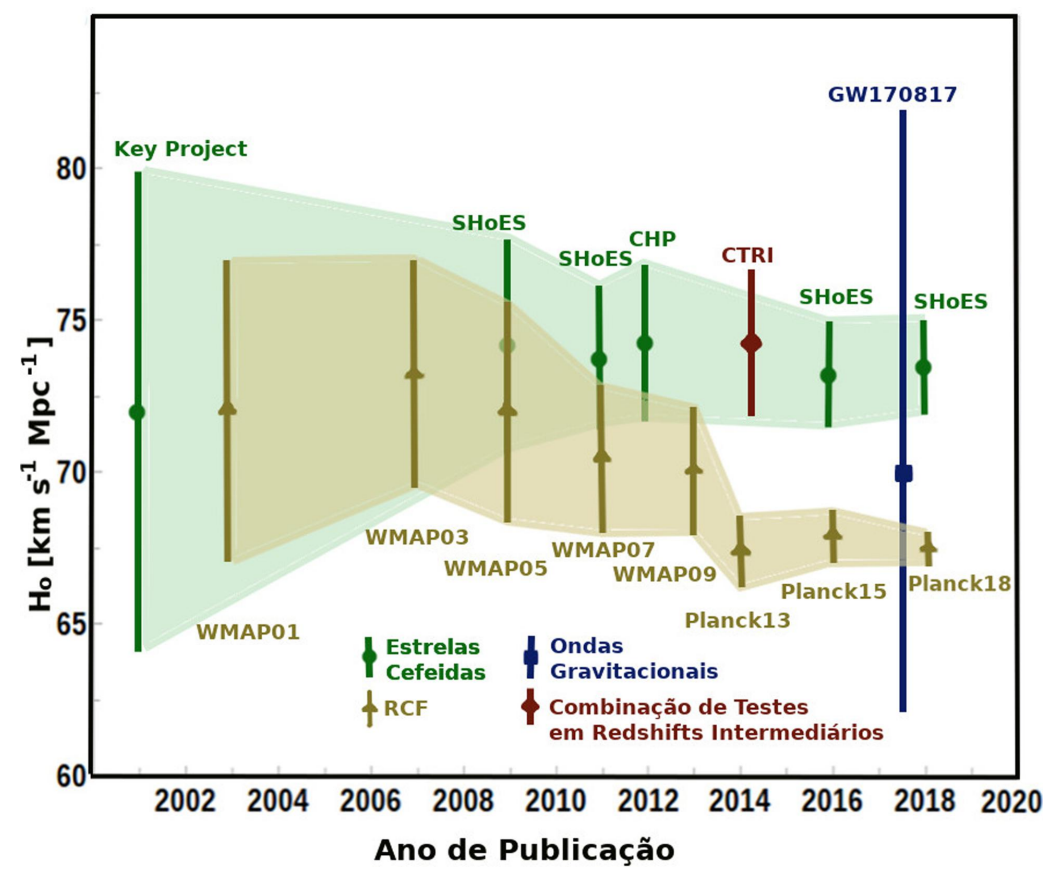

Figura 1.6: Gráfico retirado de Lima e Santos (2019), mostrando a evolução nas medidas da constante de Hubble e a crescente tensão entre os resultado via CMB (medidas em bege) e Supernovas (medidas em verde).

No capítulo a seguir, apresentaremos uma classe de modelos alternativos que se propõe a resolver alguns dos problemas mencionados nessa seção. 
Capítulo 2

\section{Modelos com Decaimento do Vácuo - $\Lambda(\mathrm{t})$}

Vistos os problemas existentes com o modelo padrão, há uma vastidão de modelos alternativos propostos. As ideias mais conservadoras sugerem modificações no setor de energia escura, indo desde descrições com $\Lambda(\mathrm{t}$ ) (Carvalho et al., 1992) que serão o tema central desse capítulo, passando por campos escalares não canônicos (e.g quintessência (Ratra e Peebles, 1988) e k-essência (Armendariz-Picon et al., 2001; Vikman, 2007) até os modelos em que o $\omega$ da equação de estado de estado varia com o tempo ou com o redshift (Yang et al., 2017). Discutiremos no capítulo a seguir os modelos com campos escalares, canônicos e não canônicos, voltados principalmente para a descrição do Universo primordial, incluindo o regime inflacionário.

Além dos citados acima, existem modelos, como o de criação de matéria escura (Prigogine et al., 1989; Lima e Germano, 1992), sugerindo modificações no tensor de energiamomento das equações de Einstein. Tais modelos são dotados de uma pressão negativa e, portanto, são capazes de justificar a expansão acelerada do Universo sem energia escura. Existem ainda modelos provenientes de teorias de gravitação modificada como, por exemplo, teorias F(R) (Bergmann, 1968; Starobinsky, 1980) e F(T) (Clifton et al., 2012).

Antes de começar a discussão sobre a classe de modelos com decaimento do vácuo, vamos ver quais são as implicações para a conservação de energia.

\subsection{Conservação de Energia nos Modelos com $\Lambda(t)$}

Vamos estudar a conservação do tensor energia-momento $T_{T}^{\mu \nu}$ como descrito pela equação (1.4): 


$$
\begin{aligned}
u_{\mu} T_{(T) ; \nu}^{\mu \nu} & =0 \\
\Rightarrow & u_{\mu} T_{(f) ; \nu}^{\mu \nu}+u_{\mu} T_{(\Lambda) ; \nu}^{\mu \nu}=0 \\
\Rightarrow & u_{\mu} T_{(f) ; \nu}^{\mu \nu}=-u_{\mu}\left(g^{\mu \nu} \frac{\Lambda_{, \nu}}{8 \pi G}+g_{; \nu}^{\mu \nu} \frac{\Lambda}{8 \pi G}\right) .
\end{aligned}
$$

Como a derivada covariante da métrica é nula, obtemos:

$$
u_{\mu} T_{(f) ; \nu}^{\mu \nu}=-u^{\nu} \frac{\Lambda_{, \nu}}{8 \pi G}
$$

Historicamente, pensou-se no vácuo desacoplado, isto é, não interagindo com a matéria. Portanto, a expressão acima tomaria a forma $T_{(f) ; \nu}^{\mu \nu}=0$, recuperando o resultado da seção 1.1.1 e fornecendo $\Lambda=$ cte. Ou seja, aqui temos um resultado geral (válido para qualquer métrica): quando não existe interação entre o vácuo e as componentes do fluido cosmológico, $\Lambda$ é uma constante.

O termo à esquerda na equação (2.1) é exatamente o termo de conservação para um fluido perfeito deduzido na equação 1.22 , já o termo à direita é uma simples derivada temporal de $\Lambda$. Assim, incluindo o termo $\Lambda$-variável, a equação se torna uma equação de balanço para a densidade de energia do fluido material:

$$
\dot{\rho_{f}}+3 H\left(\rho_{f}+p_{f}\right)=-\dot{\rho_{\Lambda}}=-\frac{\dot{\Lambda}}{8 \pi G}
$$

Essa expressão mostra que se $\Lambda$ variar com o tempo, existe uma interação entre o vácuo e as outras componentes do fluido. Mais especificamente, devido ao sinal negativo do lado esquerdo da equação, podemos interpretar o vácuo como uma fonte para as outras componentes. Inversamente, ao considerarmos $\Lambda=$ cte., necessariamente excluímos a possibilidade de interação do vácuo com o restante do fluido. Para saber como se dá essa interação, precisamos saber qual é a forma funcional de $\Lambda(t)$.

\subsection{Lei de Decaimento do Vácuo}

Nessa seção, faremos uma apresentação histórica do ansatz que utilizaremos para descrever o decaimento do vácuo. A primeira proposta dessa lei pode ser encontrada em Ozer e Taha (1986, 1987). Eles partiram de um modelo cosmológico com curvatura arbitrária 
e impuseram as condições de que a soma das densidades de energia da matéria e da radiação deveria ser igual à densidade crítica e que a variação da entropia é sempre positiva. Fazendo isso, mostra-se que esse universo é fechado $(k=1)$ com a constante cosmológica sendo definida por:

$$
\begin{aligned}
& \Lambda(a)=\frac{3}{a^{2}}, \\
& \Rightarrow \rho_{\Lambda}=\frac{3}{8 \pi G a^{2}} .
\end{aligned}
$$

Alguns anos depois, Chen e $\mathrm{Wu}(1990)$ utilizaram um argumento dimensional para justificar a validade de um modelo com $\Lambda \propto a(t)^{-2}$. Em seu trabalho, eles argumentam que é natural o decaimento ser descrito por uma quantidade adimensional multiplicada pela densidade de Planck, uma vez que essa dá a escala de energia:

$$
\frac{\Lambda(a)}{8 \pi G}=\rho_{\Lambda}(a) \propto \frac{\rho_{p l}}{8 \pi G}\left[\frac{L_{p l}}{a(t)}\right]^{n} .
$$

Nessa expressão, o fator de escala fixa a dependência temporal. A densidade e o comprimento de Planck $\rho_{p l}, L_{p l}$ são dados, respectivamente, por:

$$
\begin{aligned}
\rho_{p l} & =\frac{c^{5}}{h G^{2}}, \\
L_{p l} & =\left(\frac{\hbar G}{c^{3}}\right)^{1 / 2} .
\end{aligned}
$$

Como a relatividade geral é um teoria clássica, é esperado que a constante de Planck não esteja presente nas expressões. Portanto, para que ela seja cancelada, escolhe-se $n=2$. Assim, a equação (2.5) assume a forma:

$$
=\rho_{\Lambda}(a)=\frac{\Lambda(a)}{8 \pi G}=\frac{\gamma}{a^{2}},
$$

onde $\gamma$ é uma constante adimensional.

O modelo de Chen e Wu é um generalização do modelo de Ozer e Taha pois a expressão acima é válida para universos com qualquer curvatura. Além disso, o modelo fornece um argumento físico para sustentar a ideia de um modelo com $\Lambda(t) \propto a(t)^{-2}$. As consequências desse modelo para a dinâmica do Universo foram analisadas em Abdel-Rahman (1992). 
Posteriormente, Carvalho et al. (1992) utilizaram um argumento dimensional para propor uma generalização do modelo de Chen e Wu, considerando a lei de decaimento do tipo $\Lambda(H, a)$ :

$$
\Lambda(H, a)=3 \beta H^{2}+3 \frac{\alpha}{a^{2}},
$$

onde $\alpha$ e $\beta$ são constantes e o fator 3 foi incluído por conveniência matemática. Porém, esse modelo é descartado pois nele não existe um redshift de transição entre a fase acelerada e desacelerada (Lima e Maia, 1994; Lima e Trodden, 1996; Perico et al., 2013). Com isso em mente, foi proposta uma modificação ao modelo (Shapiro e Solà, 2002):

$$
\Lambda(t)=c_{0}+3 \beta H^{2}
$$

onde $c_{0}=8 \pi G \rho_{\Lambda 0}$ e $\beta \equiv \frac{\rho_{\Lambda}-\rho_{\Lambda 0}}{\rho_{f}+\rho_{\Lambda}}$ são constantes (Perico et al. 2013$)$.

Nesse caso, o valor da constante cosmológica atualmente é dado por $\Lambda_{0}=c_{0}+3 \beta H_{0}^{2}$. A constante $c_{0}$ resolve o problema da existência de uma fase de transição entre as fases acelerada e desacelerada. É importante chamar a atenção para o fato de que os estágios finais são do tipo $\Lambda \mathrm{CDM}$ quando $\beta \rightarrow 0$.

É importante mencionar que, no modelo acima foi utilizado $\beta$ constante, entretanto, não existe nenhum princípio fundamental que imponha tal condição. De fato, uma descrição mais geral seria $\beta \equiv \beta(t)$, em que podemos assumir a seguinte expansão para o parâmetro:

$$
\beta(t)=\nu+\alpha\left(\frac{H}{H_{I}}\right)^{n},
$$

onde $\nu, \alpha$ são constantes cujas interpretações serão esclarecidas mais à frente e $H_{I}$ é o valor do parâmetro de Hubble no início do Universo. Com essa expansão, a densidade do vácuo é expressa por:

$$
\Lambda(H)=c_{0}+3 \nu H^{2}+3 \alpha H^{2}\left(\frac{H}{H_{I}}\right)^{n} .
$$

Repare que, para o caso $\alpha \rightarrow 0$, a equação acima assume a forma de 2.10. É importante ressaltar que, dimensionalmente $[H]^{-1}=[t]$. Assim, podemos considerar que uma dependência de $\Lambda$ com o parâmetro de Hubble é equivalente a uma dependência temporal como já suposto acima. É conhecido também que $|\nu|=\mathcal{O}\left(10^{-3}\right.$ ) Basilakos et al. 2009. Grande et al., 2011), sendo uma correção pequena para a dinâmica do Universo recente. O 
caso com $c_{0}=0$ e $\nu=1-\beta$ e valores arbitrários de $n$, foi fenomenologicamente proposto em Maia (2000).

A expressão 2.12) será a lei de decaimento que iremos adotar no restante do trabalho. Vale ressaltar que, embora sua motivação original tenha sido puramente fenomenológica, essa lei de decaimento pode ser obtida via teoria quântica de campos para o caso $n=2$ (Shapiro e Solà, 2002; Solà, 2008). A seguir, investigaremos quais são as suas consequências para a dinâmica do Universo.

\subsection{Dinâmica do Modelo $\Lambda(t)$}

A dependência temporal da densidade do vácuo não modifica a forma das equações de Friedmann (1.23) e (1.24). Por isso, basta substituir nelas a lei de decaimento adotada (2.12) e seguir o mesmo procedimento descrito na seção 1.5 para obter a equação da que governa a dinâmica desse modelo (Perico et al., 2013):

$$
\dot{H}+\frac{3(1+\omega)}{2} H^{2}\left[1-\nu-\frac{c_{0}}{3 H^{2}}-\alpha\left(\frac{H}{H_{I}}\right)^{n}\right]=0 .
$$

Vimos na seção 2.1 que nessa descrição $\Lambda$ representa a densidade de energia do vácuo e age como um termo de fonte. Assim, a ideia desse modelo é que o Universo comece em um estado inicial de puro vácuo (Lima et al., 2013, 2016, Zilioti et al., 2018) e que esse vácuo seja responsável por gerar as outras componentes do fluido cosmológico, decaindo na componente dominante de cada fase. Isso significa que o modelo começa numa fase quase de Sitter (sendo, de fato, de Sitter apenas no instante inicial) e, portanto, como demonstramos na seção 1.2.1, acelerada. Além disso, sabemos que hoje o Universo também expande aceleradamente e, portanto, é também descrito por uma fase inflacionária.

Sob a luz desses dois argumentos, faremos a imposição de que o Universo comece e termine numa fase inflacionária do tipo de Sitter. Isso quer dizer que o termo entre chaves na equação (2.13) é aproximadamente nulo nos limites citados. Podemos utilizar esses limites para reduzir o número de parâmetros livres do modelo: 


$$
\begin{gathered}
H \rightarrow H_{I}: 1-\nu-\alpha \approx 0, \\
\Rightarrow \alpha \approx 1-\nu . \\
H=H_{F}<<H_{I}: 1-\nu-\frac{c_{0}}{3 H_{F}^{2}} \approx 0, \\
\Rightarrow c_{0} \approx 3(1-\nu) H_{F}^{2} .
\end{gathered}
$$

Utilizamos $H_{F}$ para denotar o parâmetro de Hubble da fase de Sitter final do Universo. O parâmetro $c_{0}$ está relacionado com o valor atual da densidade de energia do vácuo. Pela equação 2.12), temos $\Lambda_{0} \approx c_{0}+3 \nu H_{0}^{2} \approx 3 H_{F}^{2}+3 \nu\left(H_{0}^{2}-H_{F}^{2}\right)$. Os valores iniciais e finais da densidade de energia do vácuo podem ser obtidos tomando os limites adequados na equação 2.12 e são, respectivamente:

$$
\begin{aligned}
& \Lambda_{I}=3 H_{I}^{2}, \\
& \Lambda_{F}=3 H_{F}^{2} .
\end{aligned}
$$

Substituindo as expressões (2.14) acima na equação (2.13), obtemos:

$$
\dot{H}+\frac{3(1+\omega)(1-\nu)}{2} H^{2}\left[1-\left(\frac{H_{F}}{H}\right)^{2}-\left(\frac{H}{H_{I}}\right)^{n}\right]=0 .
$$

Essa é uma equação diferencial não-linear e, por esse motivo, não possui uma solução analítica. No entanto, ela pode ser resolvida analiticamente em dois limites: $H \approx H_{I}$ (Universo primordial) e $H \approx H_{F}$ (Universo atual).

\subsubsection{Universo Primordial}

No caso do universo primordial, a equação para a dinâmica do Universo assume a forma:

$$
\dot{H}+\frac{3(1+\omega)(1-\nu)}{2} H^{2}\left[1-\left(\frac{H}{H_{I}}\right)^{n}\right]=0 .
$$

A solução geral dessa equação, para uma componente qualquer do fluido é dada por (Perico et al., 2013):

$$
H=\frac{H_{I}}{\left[1+C a^{\frac{3}{2} n(1+\omega)(1-\nu)}\right]^{1 / n}},
$$


Como estamos estudando o limite válido para o Universo primordial, podemos utilizar $\omega=\frac{1}{3}$. Com isso, a solução acima pode ser reescrita como:

$$
H=\frac{H_{I}}{\left[1+C a^{2 n(1-\nu)}\right]^{1 / n}},
$$

onde $C=\frac{a_{*}^{-2 n(1-\nu)}}{(1-2 \nu)}$ é uma constante de integração que pode ser determinada considerando o final da fase de Sitter inicial. De fato, nesse momento temos $\ddot{a}=0\left(a=a_{*}\right)$ ou, equivalentemente, $\rho_{r}=\rho_{\Lambda}$, ver equações 2.22 e (2.23).

Para obter a evolução temporal do fator de escala, basta reescrever a equação 2.20 na sua forma integral:

$$
\int_{a_{*} \equiv a(t *)}^{a(t)} \frac{d a}{a}\left[1+C a^{2 n(1-\nu)}\right]^{1 / n}=H_{I} \Delta t,
$$

onde $t_{*}$ define o instante em que a inflação termina, $a_{*}$ é o fator de escala nesse momento e $\Delta t \equiv t-t_{*}$. No limite $t<<t_{*}$, ou seja, tempos próximos ao início do Universo, temos $C a^{2 n(1-\nu)}<<1$ e $H \approx H_{I}$. Portanto, o Universo inicia sua evolução de um estado quase de Sitter $a(t) \approx e^{H_{I} \Delta t}$.

Substituindo a equação (2.20) nas equações de Friedmann 1.23 e (1.24), podemos estudar a evolução das densidades de radiação e do vácuo no início do Universo, dadas respectivamente por (Perico et al., 2013):

$$
\begin{aligned}
\rho_{r} & =\rho_{I} \frac{(1-\nu) C a^{2 n(1-\nu)}}{\left[1+C a^{2 n(1-\nu)}\right]^{1+2 / n}}, \\
\rho_{\Lambda} & =\rho_{I} \frac{1+\nu C a^{2 n(1-\nu)}}{\left[1+C a^{2 n(1-\nu)}\right]^{1+2 / n}},
\end{aligned}
$$

onde $\rho_{I} \equiv \frac{H_{I}^{2}}{8 \pi G}$ é o valor da densidade de energia total inicial do Universo. Tomando o limite para o início do Universo $(a(t) \rightarrow 0)$ vemos que, efetivamente toda energia está concentrada na componente de vácuo, pois $\rho_{r} \rightarrow 0$ e $\rho_{\Lambda} \rightarrow \rho_{I}$. O gráfico 2.1 ilustra essa evolução para diferentes escolhas do parâmetro $n$. 


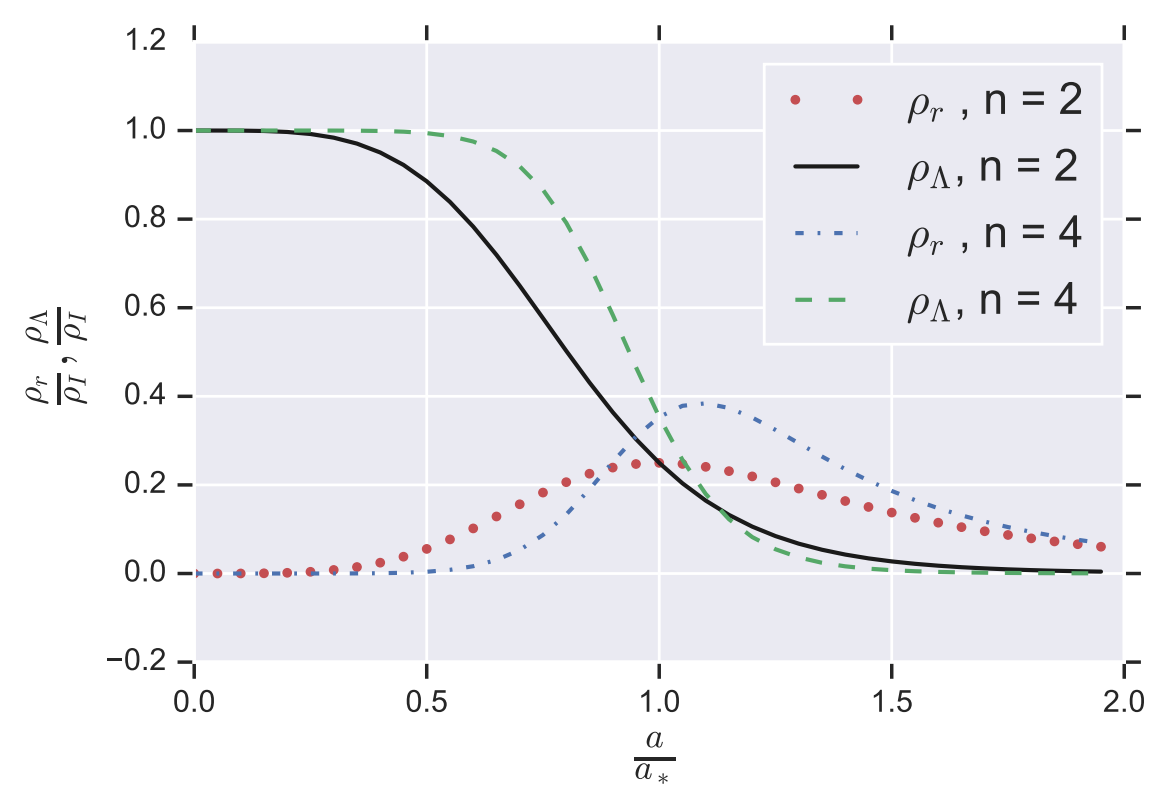

Figura 2.1: Nesse gráfico, mostramos a evolução das densidades de radiação e do vácuo com o fator de escala. Ele ilustra como o decaimento do vácuo depende do parâmetro n: ocorrendo mais tardiamente e gerando mais radiação à medida que este aumenta. É interessante ressaltar também que existe uma transição contínua da fase dominada pelo vácuo para a radiação. Para fazer o gráfico, utilizamos $\nu=10^{-3}$.

Sabemos que, durante a inflação, o fator de escala cresceu muito, portanto, discutiremos o período pós-inflacionário considerando $C a^{2 n(1-\nu)}>>1$. Neste limite, pode-se verificar que as densidades do fluido cosmológico evoluem da seguinte forma (Perico et al., 2013):

$$
\begin{aligned}
& \rho_{r} \propto(1-\nu) a^{-4(1-\nu)} \\
& \rho_{\Lambda} \propto \nu a^{-4(1-\nu)} .
\end{aligned}
$$

Vemos que, para $\nu<<1$, temos a dinâmica esperada na era da radiação. Ou seja, verificamos que existe um transição natural de uma fase inflacionária inicial dominada pelo vácuo para uma fase dominada pela radiação.

\subsubsection{Universo Atual}

No limite $H<<H_{I}$, a equação que fornece a dinâmica do modelo assume a forma:

$$
\dot{H}+\frac{3(1+\omega)(1-\nu)}{2} H^{2}\left[1-\left(\frac{H_{F}}{H}\right)^{2}\right]=0 .
$$

A solução dessa equação, para uma componente qualquer do fluido é dada por: 


$$
H=H_{F}\left[1+D a^{-3(1+\omega)(1-\nu)}\right]^{1 / 2} .
$$

Como estamos estudando o Universo recente, podemos utilizar $\omega=0$. Assim, a equação acima assume a forma obtida em Zilioti et al. (2018),

$$
H=H_{F}\left[1+D a^{-3(1-\nu)}\right]^{1 / 2},
$$

onde D é uma constante de integração.

É conveniente reescrever a equação acima em termos de parâmetros observáveis, uma vez que ela modela o Universo atual. Para isso, resolveremos a equação (2.26) em função do redshift $z$. Fazendo isso, obtém-se:

$$
H=H_{0} \sqrt{\frac{\Omega_{\Lambda 0}-\nu}{1-\nu}}\left[1+\frac{\Omega_{m 0}}{\Omega_{\Lambda 0}-\nu}(1+z)^{3(1-\nu)}\right]^{1 / 2} .
$$

Fazendo uma comparação entre as equações 2.28) e (2.29), podemos obter o valor da constante $\mathrm{D}$ e de $H_{F}$ em termos de parâmetros observáveis: $D=\frac{\Omega_{m 0}}{\Omega_{\Lambda 0}-\nu}$ e $H_{F}=H_{0} \sqrt{\frac{\Omega_{\Lambda 0}-\nu}{1-\nu}}$. Tomando $\nu \approx 0$ e $\Omega_{\Lambda 0} \approx 0$, obtemos $H_{f} \approx 0.84 H_{0}$. De fato, é esperado que $H_{F}$ seja menor do que a constante de Hubble, já que isso indica que o parâmetro de Hubble assumirá esse valor no futuro. Repare que $H_{0}=H_{F} \Leftrightarrow \Omega_{\Lambda 0}=1$, ou seja, o parâmetro de Hubble assumirá seu valor final quando o Universo for novamente dominado pelo vácuo. É interessante salientar também que todas as expressões nessa seção recuperam o modelo $\Lambda$ CDM se tomamos $\nu=0$.

Substituindo a equação 2.29 nas equações de Friedmann, podemos encontrar a evolução temporal das densidades de energia da matéria e do vácuo (Perico et al., 2013), dadas, respectivamente, por:

$$
\begin{aligned}
& \rho_{m}=\rho_{m 0} a^{-3(1-\nu)} \\
& \rho_{\Lambda}=\rho_{\Lambda 0}+\frac{\nu \rho_{m 0}}{1-\nu}\left[a^{-3(1-\nu)}-1\right] .
\end{aligned}
$$

Novamente, podemos ver que, se considerarmos $\nu=0$, recuperamos os resultados esperados pelo modelo $\Lambda$ CDM. Vemos também que, no limite $a(t) \rightarrow \infty$, a matéria é completamente diluída e o vácuo passa a dominar a dinâmica do Universo, assumindo o valor da constante cosmológica, ou seja, se comportando, efetivamente, como a energia escura. 


\subsection{Possível Solução de Alguns Problemas do Modelo Padrão}

Com esses resultados sobre a dinâmica do modelo $\Lambda(\mathrm{t})$, podemos discutir como esse modelo pretende resolver os problemas citados do $\Lambda$ CDM.

\subsubsection{Problema da Coincidência Cosmológica}

Para discutir esse problema, precisamos obter a dependência dos parâmetros de densidade desse modelo com o parâmetro de Hubble. Fazemos isso substituindo a equação para o decaimento do vácuo na definição dos parâmetros de densidade 1.26 e utilizando o fato de que $\Omega_{f}=1-\Omega_{\Lambda}$ :

$$
\begin{aligned}
& \Omega_{\Lambda}=\nu+(1-\nu)\left(\frac{H_{F}}{H}\right)^{2}+(1-\nu)\left(\frac{H^{n}}{H_{I}}\right)^{n} \\
& \Omega_{f}=1-\nu+(1-\nu)\left(\frac{H_{F}}{H}\right)^{2}+(1-\nu)\left(\frac{H^{n}}{H_{I}}\right)^{n}
\end{aligned}
$$

Os parâmetros de densidade do vácuo e do meio material são iguais em duas épocas distintas. Para mostrar isso, basta igualar as duas expressões acima,

$$
2 \nu+2(1-\nu)\left(\frac{H_{F}}{H}\right)^{2}+2(1-\nu)\left(\frac{H^{n}}{H_{I}}\right)^{n}=1,
$$

e tomar os limites para o universo primordial e o universo atual. Fazendo isso, obtemos os parâmetros de Hubble dos dois momentos de transição:

$$
\begin{aligned}
H_{*} & =H_{I}\left[\frac{(1-2 \nu)}{2(1-\nu)}\right]^{1 / n}, \\
H_{* *} & =H_{F}\left[\frac{2(1-\nu)}{(1-2 \nu)}\right]^{1 / 2} .
\end{aligned}
$$

A primeira igualdade ocorre logo após o fim inflação primordial. Tomando $\nu=0$, temos $H_{*}=\frac{H_{I}}{2^{1 / n}}$. Podemos obter o parâmetro de Hubble no momento da segunda transição, $H_{* *}$, em função de $H_{0}$. Tomando $H_{F}=0.84 H_{0}$ e $\nu=0$, temos $H_{* *}=1.18 H_{0}$, ou seja, essa transição ocorre, como esperado, em um momento anterior à hoje. Uma vez que, como sabemos, o Universo passou por uma fase dominada pela matéria e está agora em uma fase dominada pela energia escura. 

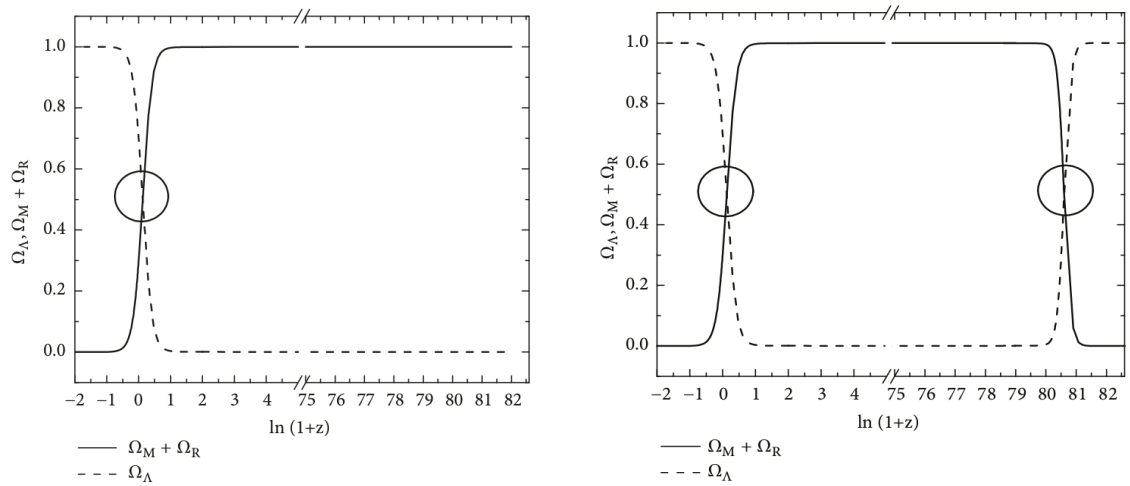

Figura 2.2: $\mathrm{O}$ gráfico à esquerda é referente ao modelo $\Lambda \mathrm{CDM}$ clássico, já o gráfico à direita é referente ao modelo de decaimento do vácuo (ambos retirados de Zilioti et al. (2018)). Nos dois gráficos, a linha pontilhada representa o parâmetro do vácuo e os círculos mostram o redshift para o qual $\Omega_{\Lambda}=\Omega_{f}$.

Nos gráficos 2.2, mostramos a evolução dos parâmetros de densidade no início e no final do Universo. A existência de dois pontos de igualdade é uma possível explicação para o problema da coincidência, pois mostra que o momento de igualdade entre as duas densidades não é um ponto único na história do Universo, tendo ocorrido ao menos uma outra vez, com a possibilidade de ser um evento cíclico.

Note que, em ambos os gráficos temos na ordenada o parâmetro de densidade do meio material total, isto é $\Omega_{f}=\Omega_{r}+\Omega_{m}$. No entanto, para o início do Universo esse parâmetro é representado apenas pela componente da radiação, enquanto no Universo atual ele é representado pela componente de matéria.

O gráfico da direita ilustra como em um modelo com decaimento do vácuo o Universo evolui de uma fase de Sitter, Essa fase é seguida por uma fase dominada pela radiação, depois para uma fase dominada pela matéria e, finalmente, volta à uma fase de Sitter final. Isso também é ilustrado ao se tomar os limites dos parâmetros de densidade no início e final do Universo:

$$
\begin{aligned}
& \lim _{H \rightarrow H_{I}} \Omega_{\Lambda}=1, \quad \lim _{H \rightarrow H_{I}} \Omega_{f}=0 . \\
& \lim _{H \rightarrow H_{F}} \Omega_{\Lambda}=1, \quad \lim _{H \rightarrow H_{F}} \Omega_{f}=0 .
\end{aligned}
$$

\subsubsection{Problema da Constante Cosmológica}

Como dissemos ao longo do texto, temos as densidades de energia inicial e final do vácuo dadas, respectivamente, por $\Lambda_{I}=3 H_{I}{ }^{2}$ e $\Lambda_{F}=3 H_{F}{ }^{2}$. Como $H_{F}<<H_{I}$, é esperado que 
a densidade de energia do vácuo atualmente seja muito menor do que a inicial. Tomando a razão da densidade inicial sobre a densidade final, obtemos:

$$
\frac{\rho_{\Lambda_{I}}}{\rho_{\Lambda_{F}}}=\frac{\Lambda_{I}}{\Lambda_{F}}=\left(\frac{H_{I}}{H_{F}}\right)^{2} .
$$

Sabemos que $H_{F} \sim H_{0} \sim 10^{-42} \mathrm{GeV}$. Então, assumindo $H_{I} \sim 10^{19} \mathrm{GeV}$ (limite de validade para teorias de gravitação clássica), temos $\rho_{\Lambda_{I}} / \rho_{\Lambda_{F}} \sim 10^{-122}$, valor consistente com estimativas baseadas na teoria quântica de campos (Weinberg, 1989, 2008b; Lima et al. 2013). Assim, se relacionarmos o valor da densidade de energia inicial do vácuo $\rho_{\Lambda_{I}}$ com os valores estimados por teorias quânticas e o valor da densidade de energia final $\rho_{\Lambda_{F}}$ com os valores medidos observacionalmente, essa poderia ser uma possível explicação para a sua disparidade. No entanto, esse cenário ainda não pode ser apresentado como uma solução efetiva a esse problema, uma vez que ainda não foi possível mostrar por primeiros princípios o valor da escala de energia de $H_{I}$.

Além de aliviar tais problemas, o modelo também oferece vantagens no que diz respeito ao cenário inflacionário, pois fornece uma explicação natural para o começo da inflação e possui uma saída graciosa, pois, como vimos, sua transição para uma fase de radiação é contínua. Por começar em uma era de Sitter o modelo também é livre do problema de horizontes e de uma singularidade inicial. Para uma discussão mais aprofundada desses problemas, ver Zilioti et al. (2018).

Dadas todas as vantagens, seria interessante confrontar as previsões desse modelo com o $\Lambda$ CDM. É esperado que as ondas gravitacionais forneçam medidas observacionais nesse sentido (Tamayo et al., 2017), embora atualmente nossa tecnologia não seja capaz de realizar as medições com a precisão necessária. Naturalmente, para que isso seja feito é necessário estudar melhor o cenário inflacionário desse modelo, sendo assim é conveniente obter uma descrição de campo escalar para o mesmo. Portanto, antes de obter essa descrição, nos aprofundaremos na discussão sobre os modelos de campo escalar e sua relação com a inflação. 


\section{Capítulo 3}

\section{Modelos com Campos Escalares: Campo Canônico e K-Essência}

Começaremos nossa discussão sobre campos escalares mostrando como podemos obter as equações de Einstein a partir da variação da ação de Einstein-Hilbert, definida por (Landau e Lifshitz, 1980):

$$
S=\int\left[-\frac{1}{16 \pi G} R+\mathcal{L}_{f}\right] \sqrt{-g} d^{4} x
$$

onde $g=\operatorname{det}\left(g^{\mu \nu}\right)$ é o determinante da métrica e $\mathcal{L}_{f}$ é a lagrangiana dos campos escalares que descrevem a presença do conteúdo energético-material do Universo. Pelo princípio de ação mínima, sabemos que a variação da ação acima dever ser nula, $\delta S=0$. Daí, obtemos (Landau e Lifshitz, 1980):

$$
\int\left[-\frac{1}{16 \pi G} \frac{\delta(\sqrt{g} R)}{\delta g^{\mu \nu}}+\frac{\delta\left(\sqrt{g} \mathcal{L}_{f}\right)}{\delta g^{\mu \nu}}\right] \delta g^{\mu \nu} d^{4} x=0 .
$$

Como essa igualdade deve ser válida para qualquer variação $\delta$, o termo entre chaves deve ser identicamente nulo,

$$
-\frac{1}{16 \pi G} \frac{\delta(\sqrt{g} R)}{\delta g^{\mu \nu}}+\frac{\delta\left(\sqrt{g} \mathcal{L}_{f}\right)}{\delta g^{\mu \nu}}=0 .
$$

Reorganizando a equação acima e efetuando a variação nos termos geométricos, temos a equação de movimento para esse campo,

onde definimos $M_{P}=\frac{1}{\sqrt{8 \pi G}}$.

$$
\frac{\delta R}{\delta g^{\mu \nu}}+\frac{R}{\sqrt{-g}} \frac{\delta \sqrt{-g}}{\delta g^{\mu \nu}}=M_{P}^{2} \frac{2}{\sqrt{-g}} \frac{\delta\left(\sqrt{g} \mathcal{L}_{f}\right)}{\delta g^{\mu \nu}} .
$$


É possível mostrar que o lado esquerdo dessa equação fornecerá os termos geométricos da equação de Einstein (Carroll, 2004),

$$
\frac{\delta R}{\delta g^{\mu \nu}}+\frac{R}{\sqrt{-g}} \frac{\delta \sqrt{-g}}{\delta g^{\mu \nu}}=R_{\mu \nu}-\frac{1}{2} g_{\mu \nu} R
$$

Assim, definindo o tensor energia-momento como o lado direito da equação (3.4),

$$
T_{\mu \nu}^{f} \equiv \frac{2}{\sqrt{-g}} \frac{\delta\left(\sqrt{g} \mathcal{L}_{f}\right)}{\delta g^{\mu \nu}}=-g_{\mu \nu} \mathcal{L}_{f}+2 \frac{\delta \mathcal{L}_{f}}{\delta g^{\mu \nu}}
$$

obtemos a equação de Einstein:

$$
R_{\mu \nu}-\frac{1}{2} g_{\mu \nu} R=M_{P}^{2} T_{\mu \nu}^{f}
$$

Fornecendo uma forma para a lagrangiana do fluido $\mathcal{L}_{f}$, definimos o tensor energiamomento e, daí podemos obter as equações dinâmicas para o modelo cosmológico adotado.

Nessa dedução, suprimimos a constante cosmológica. Para incluí-la, bastaria adicionar um termo constante ao tensor de Ricci na ação de Einstein-Hilbert. Alternativamente, $\Lambda$ também pode ser incluída no tensor de energia-momento como a componente que descreve o vácuo. Se fornecemos uma forma para a densidade langragena $\mathcal{L}_{f}$, é possível obter as densidades e pressão do fluido. Com isso, temos todos os ingredientes necessários para obter as equações de Friedmann nessa descrição e resolvê-las.

\subsection{Dinâmica Cosmológica para o Campo Escalar Canônico}

Nosso interesse nessa seção é mostrar como podemos obter as equações de Friedmann para um campo escalar. A lagrangiana mais simples que podemos escolher é a chamada lagrangiana do campo canônico:

$$
\mathcal{L}_{f}=\frac{1}{2} \partial^{\mu} \phi \partial_{\mu} \phi-V(\phi)
$$

onde o primeiro termo se relaciona com uma espécie de energia cinética do campo $\phi$ e o segundo com o potencial. Substituindo a lagrangiana acima em (3.6), podemos obter o seu tensor energia-momento: 


$$
\begin{aligned}
T^{\mu \nu} & =-g^{\mu \nu} X+g^{\mu \nu} V(\phi)+\partial^{\mu} \phi \partial^{\nu} \phi, \\
& =\frac{1}{2} \partial^{\mu} \phi \partial^{\nu} \phi+g^{\mu \nu} V(\phi) .
\end{aligned}
$$

Como os modelos cosmológicos FLRW são homogêneos e isotrópicos, fazemos a escolha $\phi \equiv \phi(t)$. Com essa imposição, a densidade de energia e pressão do campo são dadas por:

$$
\begin{gathered}
\rho_{\phi}=T^{00}=\frac{\dot{\phi}^{2}}{2}+V(\phi), \\
p_{\phi}=T_{j}^{i}=\frac{\dot{\phi}^{2}}{2}-V(\phi) .
\end{gathered}
$$

Substituindo a expressão para o tensor energia-momento (3.9) em (2.7), podemos obter as duas equações de Friedmann nessa descrição:

$$
\begin{aligned}
3 M_{P}^{2} H^{2} & =\frac{\dot{\phi}^{2}}{2}+V(\phi), \\
-2 M_{P}^{2} \dot{H}-3 M_{P}^{2} H^{2} & =\frac{\dot{\phi}^{2}}{2}-V(\phi) .
\end{aligned}
$$

Repare que essas são exatamente as equações (1.43) e (1.44), assim todo o tratamento que foi descrito na seção (1.4.1) se aplica ao campo escalar canônico. Aqui, apenas demonstramos como obter essa descrição de primeiros princípios.

É comum supor que o campo escalar descreve uma fluido perfeito com EOS $p=\omega \rho$. Assim, podemos definir definir $\omega$ por meio da razão entre a sua densidade e pressão:

$$
\omega=\frac{p_{\phi}}{\rho_{\phi}}=\frac{\frac{\dot{\phi}^{2}}{2}-V(\phi)}{\frac{\dot{\phi}^{2}}{2}+V(\phi)} .
$$

No limite $\frac{\dot{\phi}^{2}}{2}<<V(\phi)$, a quintessência assumirá a EoS do vácuo, enquanto no limite $\frac{\dot{\phi}^{2}}{2}>>(\phi)$ ela assumiria a EoS da matéria rígida, ou seja, temos $\omega=[-1,1]$. Portanto, se houver uma transformação de energia potencial em cinética (ou vice-versa) no campo, a quintessência pode ser usada para descrever modelos em que a equação de estado do vácuo evolui com o tempo.

\subsection{O Caso Geral: Formulação Lagrangiana de Horndeski}

A ação de Einstein-Hilbert é escolhida com o objetivo de se obter as equações de Einstein, ou seja, é uma ação para obter as equações de movimento em RG. No entanto, 
essa não é a única ação possível. Na verdade não precisamos nem nos limitar à teoria da relatividade geral, de forma que, hipoteticamente, seria possível escolher uma ação qualquer. É claro que hipóteses físicas (e matemáticas) limitam essa escolha, tendo sido demonstrado que a ação mais geral que fornece equações de movimento de segunda ordem é dada por (Horndeski, 1974; Kase e Tsujikawa, 2019):

$$
S_{H}=\int \sqrt{-g} \mathcal{L}_{H} d^{4} x
$$

sendo,

$$
\begin{aligned}
\mathcal{L}_{H} & =G_{2}(\phi, X)+G_{3}(\phi, X) \square \phi+G_{4}(\phi, X) R \\
& +G_{4, X}(\phi, X)\left[(\square \phi)^{2}-\left(\partial_{\mu} \partial_{\nu} \phi\right)\left(\partial^{\mu} \partial^{\nu} \phi\right)\right] \\
& +G_{5}(\phi, X) G_{\mu \nu} \partial^{\mu} \partial^{\nu} \phi-\frac{1}{6} G_{5, X}(\phi, X)\left[(\square \phi)^{3}-3(\square \phi)\left(\partial_{\mu} \partial_{\nu} \phi\right)\left(\partial^{\mu} \partial^{\nu} \phi\right)\right. \\
& \left.+2\left(\partial^{\mu} \partial_{\alpha} \phi\right)\left(\partial^{\alpha} \partial_{\beta} \phi\right)\left(\partial^{\beta} \partial_{\mu} \phi\right)\right] .
\end{aligned}
$$

Na expressão acima, utilizamos $\square \equiv \partial^{\mu} \partial_{\mu}$ para indicar o operador D'lembertiano, definimos $X \equiv \frac{1}{2} \partial^{\mu} \phi \partial_{\mu} \phi$, sendo este o termo cinético canônico para campos escalares e $G_{i}$ são funções de X e $\phi$.

Fazendo escolhas sobre as funções $G_{i}$, podemos obter diferentes teorias para a dinâmica do campo escalar $\phi$. Por exemplo, supondo uma descrição sem fluido, a ação de Einstein Hilbert poder ser obtida tomando $G_{2}=G_{4}=G_{5}=0$ e $G_{3}=-\frac{R}{16 \pi G}$. Em se tratando de modelos alternativos, essa ação descreve também generalizações da RG como teorias $\mathrm{F}(\mathrm{R})$, onde a ação é dada por (Bergmann, 1968):

$$
S_{H}=\int\left[\sqrt{-g} \frac{M_{P}^{2}}{2} f(R)\right] d^{4} x,
$$

onde $f(R)$ é uma função arbitrária do tensor de Ricci. Essa ação pode ser obtida fazendo as seguintes escolhas na lagrangiana (3.15),

$$
G_{2}=-\frac{M_{P}^{2}}{2}(R F-f), \quad G_{3}=0, \quad G_{4}=\frac{M_{P}^{2}}{2} F, \quad G_{5}=0
$$

onde $F=\partial f / \partial R$. 
Para uma discussão completa sobre como obter outras teorias de gravitação por meio dessa ação, ver Kase e Tsujikawa (2019). Nos concentraremos agora nas descrições de campo escalar da quintessência e k-essência a partir da lagrangiana (3.15).

\subsubsection{Quintessência}

Para obter a descrição de quintessência, escolhemos:

$$
G_{2}=X-V(\phi), \quad G_{3}=0, \quad G_{4}=\frac{M_{P}^{2}}{2}, \quad G_{5}=0
$$

onde $V(\phi)$ é um potencial do campo $\phi$. Justamente por utilizar X como seu termo cinético, essa descrição também é conhecida como descrição de campo escalar canônica.

Sabemos que o termo $\frac{M_{P}^{2}}{2} R$ é responsável pelo lado geométrico da equação de Einstein. Por isso, vamos associar a função $G_{2}$ com uma lagrangiana do campo escalar $\phi, \mathcal{L}_{\phi}$. A ação total nesse caso pode ser escrita na forma da eq. (3.1) se definirmos $\mathcal{L}_{T} \equiv \mathcal{L}_{\phi}+\mathcal{L}_{f}$, onde $\mathcal{L}_{f}$ é a lagrangiana do fluido material, cujo tensor energia-momento é dado por 1.16 .

O tensor energia-momento do campo escalar é o mesmo obtido na seção 3.1, uma vez que ambas são a mesma descrição. Por esse motivo, não repetiremos o procedimento descrito anteriormente.

\subsubsection{K-Essência}

Para obter a descrição de k-essência, escolhemos (Kase e Tsujikawa, 2019):

$$
G_{2}=G_{2}(\phi, X), \quad G_{3}=0, \quad G_{4}=\frac{M_{P}^{2}}{2}, \quad G_{5}=0
$$

Segue daí, que a k-essência pode ser interpretada como uma generalização da quintessência, sendo esta descrita pela escolha particular da função $G_{2}(\phi, X)=X-V(\phi)$.

Podemos fazer uma associação da função $G_{2}(\phi, X)$, com uma lagrangiana $\mathcal{L}_{\phi}$, da mesma forma que fizemos para a quintessência e, em seguida, seguir o procedimento descrito anteriormente para se estudar a dinâmica de um Universo cuja componente do vácuo é descrita pela k-essência. É possível estudar condições sobre esta forma geral da k-essência para que ela descreva um cenário inflacionário adequado (Armendariz-Picon et al., 1999, 2001; Chiba et al., 2009). 
No entanto, para se obter uma descrição completa da dinâmica, é necessário escolher uma forma para a lagrangiana $\mathcal{L}_{\phi}$. Existem várias propostas para a forma dessa lagrangiana, mas nos concentraremos no caso descrito por (Mukhanov e Vikman, 2006; Unnikrishnan et al., 2012; Sebastiani et al., 2017):

$$
\mathcal{L}_{\phi}=\alpha X\left(\frac{X}{M^{4}}\right)^{\beta-1}-V(\phi)
$$

onde os parâmetros livres $(\alpha, \beta)$ assumem valores reais e são adimensionais, $\mathrm{M}$ possui dimensão de massa e $[X] \equiv\left[M^{4}\right]$. Repare que a escolha $\alpha=\beta=1$ reduz a expressão acima ao caso canônico. Não faremos nenhuma suposição para o potencial $V(\phi)$ neste momento. O leitor interessado pode encontrar diversos modelos diferentes para o potencial nas referências citadas acima. Como veremos adiante, $V(\phi)$ será utilizado para fazer uma descrição do decaimento do vácuo.

Substituindo a lagrangiana acima na equação do tensor energia-momento (3.6), obtemos:

$$
T_{\phi}^{\mu \nu}=-g^{\mu \nu} \alpha \frac{X^{\beta}}{M^{4(\beta-1)}}+g^{\mu \nu} V(\phi)+\alpha \beta\left(\frac{X}{M^{4}}\right)^{\beta-1} \partial^{\mu} \phi \partial^{\nu} \phi .
$$

Fazendo a imposição $\phi \equiv \phi(t)$, o tensor é dado por:

$$
T_{\phi}^{\mu \nu}=-g^{\mu \nu} \frac{\alpha}{M^{4(\beta-1)}}\left(\frac{\dot{\phi}^{2}}{2}\right)^{\beta}+g^{\mu \nu} V(\phi)+\alpha \beta\left(\frac{\dot{\phi}^{2}}{2 M^{4}}\right)^{\beta-1} \partial^{\mu} \phi \partial^{\nu} \phi .
$$

Sendo a densidade de energia e pressão do campo:

$$
\begin{gathered}
\rho_{\phi}=T^{00}=\frac{\alpha(2 \beta-1)}{M^{4(\beta-1)}}\left(\frac{\dot{\phi}^{2}}{2}\right)^{\beta}+V(\phi), \\
p_{\phi}=T_{j}^{i}=\frac{\alpha}{M^{4(\beta-1)}}\left(\frac{\dot{\phi}^{2}}{2}\right)^{\beta}-V(\phi) .
\end{gathered}
$$

Como seria esperado, para $\alpha=\beta=1$ as relações $(3.9)$ do caso canônico são recuperadas. Supondo que o Universo é dominado pela k-essência, podemos substituir as expressões acima nas equações de Friedmann (1.23) e (1.24) para obter a dinâmica do modelo: 


$$
\begin{aligned}
\frac{\alpha(2 \beta-1)}{M^{4(\beta-1)}}\left(\frac{\dot{\phi}^{2}}{2}\right)^{\beta}+V(\phi) & =3 M_{P}^{2} H^{2}, \\
\frac{\alpha}{M^{4(\beta-1)}}\left(\frac{\dot{\phi}^{2}}{2}\right)^{\beta}-V(\phi) & =-M_{P}^{2}\left(2 \dot{H}+3 H^{2}\right) .
\end{aligned}
$$

Pela equação de conservação de energia (1.22), obtemos a equação de movimento do campo escalar (Unnikrishnan et al. 2012)1,

$$
\ddot{\phi}+\frac{3 H \dot{\phi}}{2 \beta-1}+\left(\frac{V^{\prime}}{\alpha \beta(2 \beta-1)}\right)\left(\frac{2 M^{4}}{\dot{\phi}^{2}}\right)^{\beta-1}=0 .
$$

Note que para a escolha $\alpha=\beta=1$, todas as equações dessa seção se reduzem ao modelo de quintessência, como seria esperado.

É possível adotar as definições para os parâmetros de rolamento lento fornecidas na seção 1.4 e obtê-los para esse modelo. Assim, definindo o potencial $V(\phi)$, temos tudo que precisamos para estudar a inflação dirigida pela k-essência. O leitor pode encontrar um estudo mais detalhado, incluindo vários modelos de potencial, nas referências indicadas ao longo dessa seção.

No capítulo a seguir, apresentaremos a parte original dessa dissertação, onde utilizaremos a k-essência para descrever o modelo de decaimento do vácuo estudado anteriormente.

\footnotetext{
${ }^{1}$ Tomando $\alpha=1$ recuperamos a equação (2.17) do artigo referido. É importante ressaltar que onde eles utilizaram $\alpha$, na nossa notação, temos $\beta$.
} 
Capítulo 4

\section{K-Essência e Decaimento do Vácuo}

Nesse capítulo, apresentaremos a parte original do nosso trabalho, envolvendo uma descrição de campo escalar para o decaimento do vácuo (Lima e Almeida, 2020). Embora esse tipo de descrição já seja conhecida (ver, por exemplo, Maia e Lima (2002), Santos e Moraes (2018) ) a literatura consultada sugere que nossa abordagem utilizando k-essência em conjunto com uma interpretação não habitual do seu significado é nova.

\subsection{Campo Escalar Canônico como Decaimento do Vácuo}

Com o objetivo de ajudar na compreensão do leitor, faremos uma discussão preliminar para o caso canônico. Além disso, como não utilizamos a interpretação usual na literatura sobre o que o campo escalar descreve, é importante discuti-la antes de nos aprofundarmos na construção do modelo de decaimento do vácuo utilizando campos escalares e, em particular, a k-essência.

\subsubsection{Interpretação do Campo escalar como Decaimento do Vácuo}

Sabemos que para determinar a conservação de energia do campo canônico, basta substituir as equações para a pressão e densidade de energia do campo (3.9) na equação de conservação de energia 1.22 :

$$
\dot{\rho_{\phi}}+3 H\left(\rho_{\phi}+p_{\phi}\right) \Leftrightarrow \frac{d}{d t}\left(\frac{\dot{\phi}^{2}}{2}\right)+\frac{d V}{d t}+3 H\left(\dot{\phi}^{2}\right)=0 .
$$

De fato, no capítulo anterior, vimos que, dessa forma podemos obter a equação de movimento do campo escalar, mas esse não é nosso objetivo no momento. Essa discussão servirá como motivação para a interpretação que faremos à respeito do campo escalar. 
Passando o termo do potencial $V(\phi)$ para o lado direito da igualdade, ficamos com uma equação de balanço de energia, onde o potencial interage com o termo cinético, agindo como um termo de fonte:

$$
\frac{d}{d t}\left(\frac{\dot{\phi}^{2}}{2}\right)+3 H \dot{\phi}^{2}=-\frac{d V}{d t} .
$$

Note que essa equação é completamente análoga à equação (2.3). Isso nos leva a concluir que podemos interpretar o potencial como uma densidade de energia do vácuo e o termo cinético como uma densidade de energia de um fluido com $\operatorname{EoS} p_{s}=\rho_{s}$ (matéria rígida).

$$
\begin{aligned}
& \rho_{\Lambda}=V(\phi), \quad p_{\Lambda}=-V(\phi), \\
& \rho_{s}=\frac{\dot{\phi}^{2}}{2}, \quad p_{s}=\frac{\dot{\phi}^{2}}{2} \text {. }
\end{aligned}
$$

Embora essa não seja a interpretação habitual, ela pode ser encontrada em outros trabalhos na literatura (Carneiro e Tavakol, 2009). Isso significa que, nessa interpretação, a densidade total do fluido cosmológico é $\rho_{T}=\rho_{s}+\rho_{\Lambda}$ e a pressão total $p_{T}=p_{s}+p_{\Lambda}$. Nesse caso, a equação para o balanço de energia assume a seguinte forma:

$$
\dot{\rho_{s}}+6 H \rho_{s}=-\dot{\rho_{\Lambda}}
$$

Em outras palavras, podemos interpretar o campo escalar canônico como uma descrição do decaimento do vácuo em matéria rígida. Vamos, portanto estudar a dinâmica do universo para essa descrição.

\subsubsection{Dinâmica Cosmológica para o Campo Escalar Canônico}

Podemos utilizar as equações (3.25) $\operatorname{com} \alpha=\beta=1$ para obter as equações dinâmicas do fluido cosmológico descrito por um campo escalar canônico:

$$
\begin{aligned}
& \frac{\dot{\phi}^{2}}{2}+V(H(\phi))=3 M_{P}^{2} H^{2} \\
& \frac{\dot{\phi}^{2}}{2}-V(H(\phi))=-M_{P}^{2}\left(2 \dot{H}+3 H^{2}\right) .
\end{aligned}
$$


Como as equações do campo dependem apenas de $H=H(\phi)$, podemos supor que $H(\phi)$ define a dependência do potencial com o campo escalar. Somando e subtraindo as equações acima obtemos, respectivamente:

$$
\begin{aligned}
\dot{\phi}^{2} & =-2 M_{P}^{2} \dot{H} \\
V & =M_{P}^{2}\left(\dot{H}+3 H^{2}\right) .
\end{aligned}
$$

Para resolver esse par de equações, precisamos de um modelo para a densidade de energia do potencial $V(H)$. Com isso basta substituir o modelo na equação (4.7) para obter a equação que governa a dinâmica do universo e, em seguida, inserir esse resultado na equação 4.6 para obter a forma explícita de $\phi(H)$ (e $H(\phi)$ desde que a função seja inversível). Assim, podemos obter o potencial $V(\phi)$ e utilizá-lo para estudar o cenário inflacionário desse modelo.

Note que esse é um procedimento geral para obter a dinâmica de um universo descrito pelo campo escalar canônico, dado o potencial $V(\phi)$.

\subsubsection{Campo escalar e o Universo Primordial em Cosmologias com Decaimento do Vácuo}

Como vimos acima, podemos interpretar o potencial $V(H)$ como a densidade de energia do vácuo. Portanto, utilizaremos o modelo de decaimento do vácuo 2.12 com as aproximações adequadas para descrever o Universo Primordial, $\nu=c_{0} \approx 0$. Temos:

$$
\rho_{\Lambda}=M_{P}^{2} \Lambda=V(H)=3 M_{P}^{2} H^{2}\left(\frac{H}{H_{I}}\right)^{n}
$$

Faremos, a seguir, o tratamento descrito na seção anterior para obter a dinâmica e as densidades de energia desse modelo. Começamos substituindo a equação 4.10 em (4.9) para determinar a dinâmica:

$$
\dot{H}+3 H^{2}\left[1-\left(\frac{H}{H_{I}}\right)^{n}\right]=0 .
$$

Substituindo a o valor de $\dot{H}$ da expressão acima em 4.8 , obtemos a seguinte equação diferencial: 


$$
\dot{\phi}^{2}=6 M_{P}^{2} H^{2}\left[1-\left(\frac{H}{H_{I}}\right)^{n}\right]
$$

ou ainda,

$$
\frac{d \phi}{d H}= \pm \sqrt{\frac{2}{3}} M_{P}\left\{H^{2}\left[1-\left(\frac{H}{H_{I}}\right)^{n}\right]\right\}^{-1 / 2},
$$

onde utilizamos $\dot{\phi}=\frac{d \phi}{d H} \dot{H}$ no segundo passo. Essa equação pode escrita na forma integral:

$$
\phi-\phi_{I}= \pm \sqrt{\frac{2}{3}} M_{P} \int_{H_{I}}^{H(t)} \frac{d H}{\left\{H^{2}\left[1-\left(\frac{H}{H_{I}}\right)^{n}\right]\right\}^{1 / 2}}
$$

O leitor pode comparar essa equação com a equação (28) de Maia e Lima (2002). As duas assumem a mesma forma se escolhemos $x=1, \gamma=2$ e $\Lambda=\frac{V(H)}{M_{P}^{2}}$. Essa analogia está em total acordo com as interpretações feitas no presente trabalho: sob nossas hipóteses é esperado que esses sejam os valores adotados para os parâmetros mencionados acima. Dessa forma, podemos dizer que as duas descrições são equivalentes.

Efetuando a integração acima, obtemos o seguinte resultado:

$$
\begin{aligned}
\phi(H)-\phi_{I} & =\mp \frac{1}{n} \sqrt{\frac{2}{3}} M_{P} \ln \left(\frac{1-\sqrt{1-\left(\frac{H}{H_{I}}\right)^{n}}}{1+\sqrt{1-\left(\frac{H}{H_{I}}\right)^{n}}}\right), \\
H(\phi) & =H_{I} \operatorname{sech}^{\frac{2}{n}}\left(\sigma_{n} \phi\right),
\end{aligned}
$$

onde $\sigma_{n}=\frac{\sqrt{6} n}{M_{P}}$.

Podemos escolher $\phi_{I}=0$ sem perda de generalidade, pois seria possível redefinir $\phi$ de forma que seu valor inicial fosse igual à zero. Vamos descartar o sinal negativo na equação (4.14), pois $\phi>\phi_{I}$. Utilizamos relações das funções hiperbólicas para obter $H(\phi)$. Com essas soluções e a equação da dinâmica do modelo, podemos obter as densidades de energia do vácuo e da matéria rígida:

$$
\begin{gathered}
\rho_{\Lambda} \equiv V(\phi)=\rho_{I} \operatorname{sech}^{2[(n+2) / n]}\left(\sigma_{n} \phi\right), \\
\rho_{s} \equiv \frac{\dot{\phi}^{2}}{2}=\rho_{I} \frac{\operatorname{senh}^{4}\left(\sigma_{n} \phi\right)}{\cosh ^{2[(n+2) / n]}\left(\sigma_{n} \phi\right)} .
\end{gathered}
$$


Como um exercício, podemos estudar o valor das densidades iniciais do fluido cosmológico. Para isso, tomaremos o limite das densidades de energia acima quando $\phi \rightarrow 0$,

$$
\begin{aligned}
& \lim _{\phi \rightarrow 0} \rho_{\Lambda}=\rho_{I} \lim _{\phi \rightarrow 0} \operatorname{sech}^{2[(n+2) / n]}\left(\sigma_{n} \phi\right)=\rho_{I}, \\
& \lim _{\phi \rightarrow 0} \rho_{s}=\rho_{I} \lim _{\phi \rightarrow 0} \frac{\operatorname{senh}^{4}\left(\sigma_{n} \phi\right)}{\cosh ^{2[(n+2) / n]}\left(\sigma_{n} \phi\right)}=0 .
\end{aligned}
$$

As equações acima nos mostram que, de fato, o universo começa em uma era de Sitter, pois a densidade de energia inicial do vácuo é a densidade de energia inicial (ou da energia total) do universo e a densidade de energia da matéria rígida é zero. Nos gráficos abaixo podemos ter uma ideia melhor de como se dá a evolução das densidades com o campo $\phi$.
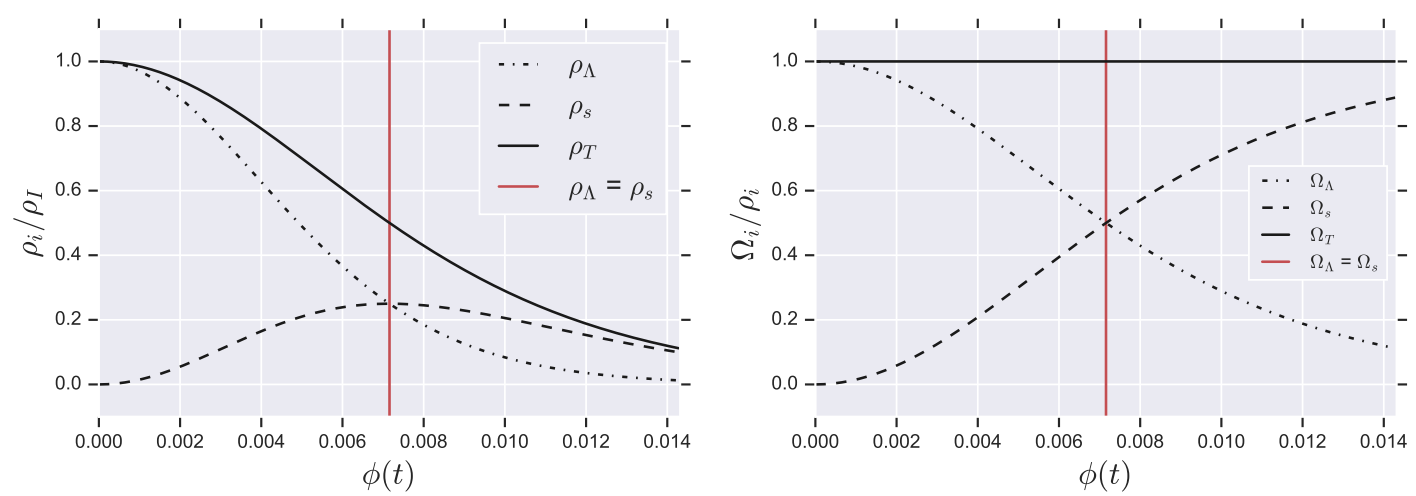

Figura 4.1: No gráfico à esquerda está representado a evolução das densidades com o campo escalar, à direita temos a evolução dos parâmetros de densidade. Escolhemos o modelo $n=2$ e unidades naturais. A linha vertical em vermelho marca o tempo em que as densidades de energia do vácuo e da matéria rígida se tornam os mesmos.

Vemos que, à medida que o tempo passa, a densidade de energia do vácuo decresce, enquanto a densidade de energia da matéria rígida aumenta até o momento em que elas se tornam iguais. A partir daí a densidade de energia da matéria também começa a decrescer. Note, entretanto, que o parâmetro de densidade da matéria continua maior do que o parâmetro de densidade do vácuo. Isso mostra que existe uma transição contínua da fase dominada pelo vácuo para a fase dominada pela matéria.

\subsection{K-Essência como Decaimento do Vácuo}

Agora que já vimos que essa interpretação do campo escalar pode nos ajudar a descrever o universo primordial, vamos fazer uma generalização para que ele seja capaz de descrever 
um fluido perfeito uma vez que, com a descrição de campo canônico, só conseguimos estudar o decaimento do vácuo em matéria-rígida, sendo essa uma componente não tradicional em modelos cosmológicos e que exigiria uma nova transição de fase para a era da radiação. Para isso utilizaremos K-essência, descrita no capítulo anterior.

\subsubsection{K-Essência como um Fluido Perfeito}

Para explicar como podemos utilizar a k-essência para descrever um fluido perfeito, vamos começar estudando o caso em que o potencial é nulo, $V(\phi)=0$. Nessa forma, as equações para a densidade e pressão do campo escalar (3.23) se reduzem à:

$$
\begin{aligned}
& \rho_{\phi}=T^{00}=\frac{\alpha(2 \beta-1)}{M^{4(\beta-1)}}\left(\frac{\dot{\phi}^{2}}{2}\right)^{\beta}, \\
& p_{\phi}=T_{j}^{i}=\frac{\alpha}{M^{4(\beta-1)}}\left(\frac{\dot{\phi}^{2}}{2}\right)^{\beta} .
\end{aligned}
$$

Queremos que o termo cinético descreva um fluido perfeito com EoS $p=\omega \rho$. Para isso, escolhemos $\beta=\omega$ e $\alpha(2 \beta-1)=1$. Assim, obtemos as seguintes expressões para a pressão e densidade de energia do fluido:

$$
\begin{aligned}
& \rho_{\phi}=M^{4}\left(\frac{\dot{\phi}^{2}}{2 M^{4}}\right)^{\frac{1+\omega}{2 \omega}}, \\
& p_{\phi}=\omega M^{4}\left(\frac{\dot{\phi}^{2}}{2 M^{4}}\right)^{\frac{1+\omega}{2 \omega}}=\omega \rho_{\phi} .
\end{aligned}
$$

Com isso, conseguimos obter uma descrição de fluido perfeito utilizando a k-essência. É importante salientar que essa descrição vale para $\omega$ no intervalo $[-1,1]$ a menos do ponto $\omega=0$, devido à nossa escolha para o parâmetro $\alpha$. Isso significa que não podemos descrever a fase da matéria com esse campo.

Agora podemos incluir novamente o potencial $V(\phi)$ ou, equivalentemente, a densidade de energia do vácuo, $\rho_{\Lambda}$. Temos: 


$$
\begin{array}{r}
\rho_{\phi}=M^{4}\left(\frac{\dot{\phi}^{2}}{2 M^{4}}\right)^{\frac{1+\omega}{2 \omega}}+V(\phi), \\
p_{\phi}=\omega M^{4}\left(\frac{\dot{\phi}^{2}}{2 M^{4}}\right)^{\frac{1+\omega}{2 \omega}}-V(\phi) .
\end{array}
$$

Esse par de equações nos permite interpretar a densidade (pressão) do campo escalar como uma soma das densidades (pressões) de dois fluidos perfeitos, de maneira análoga à que fizemos para o campo canônico. Nesse caso, temos:

$$
\begin{aligned}
\rho_{\Lambda} & =V(\phi), & p_{\Lambda} & =-V(\phi), \\
\rho_{\omega} & =M^{4}\left(\frac{\dot{\phi}^{2}}{2 M^{4}}\right)^{\frac{1+\omega}{2 \omega}}, & p_{\omega} & =\omega M^{4}\left(\frac{\dot{\phi}^{2}}{2 M^{4}}\right)^{\frac{1+\omega}{2 \omega}}=\omega \rho_{\phi} .
\end{aligned}
$$

Note que, para $\omega=1$ recuperamos as equações de pressão e densidade para o campo canônico (3.9) e que, utilizando essa interpretação, podemos recuperar exatamente a equação para o balanço de energia (2.3) obtida para o decaimento do vácuo.

Essa interpretação nos permite fazer uma descrição de campo escalar para o decaimento do vácuo em um fluido perfeito.

\subsubsection{Dinâmica Cosmológica para a K-Essência}

De posse dos resultados acima, podemos estudar a dinâmica do Universo na descrição de k-essência. Somando e subtraindo as equações de Friedmann para a k-essência (3.24), obtemos respectivamente:

$$
\begin{array}{r}
(1+\omega) M^{4}\left(\frac{\dot{\phi}}{2 M^{4}}\right)^{\frac{1+\omega}{\omega}}=-2 M_{P}^{2} \dot{H}, \\
(1+\omega) V(\phi)=2 M_{P}^{2} \dot{H}+3(1+\omega) M_{P}^{2} H^{2} .
\end{array}
$$

Substituindo 4.28 em 4.29 e utilizando $\dot{\phi}=\dot{H} \frac{d \phi}{d H}$, podemos obter $\phi(H)$ através da seguinte integral:

$$
\phi(H)=C_{\omega} \int_{H_{I}}^{H(t)} \frac{d H}{\left[3 M_{P}^{2} H^{2}-V(H)\right]^{\frac{1}{1+\omega}}},
$$


onde $C_{\omega}=\frac{2 \sqrt{2} M_{P}^{2} M^{\frac{1-\omega}{1+\omega}}}{1+\omega}$ e já assumimos $\phi>\phi_{I}=0$. Novamente, essa integral se reduz à integral (4.14), de forma que ela também recupera a equação (28) de Maia e Lima (2002), sob as condições descritas anteriormente.

Finalmente, dado um potencial $V(H)$, podemos obter $H(\phi)$ se a solução da integral acima for inversível e, em seguida, $V(\phi)$. Isso nos permitiria estudar não só a dinâmica do universo nesse modelo, mas também sua inflação.

\subsubsection{Universo Primordial na Descrição de Campo Escalar}

Como queremos estudar o universo primordial, estamos na era da radiação, ou seja, temos $\omega=1 / 3$. Além disso, utilizaremos o potencial 4.10. Nessas condições, o campo é dado por:

$$
\phi(H)= \pm\left(\frac{3 M_{P}^{2} M^{4}}{4 H_{I}^{2}}\right)^{\frac{1}{4}} \int_{1}^{H(t) / H_{I}} \frac{d\left(H / H_{I}\right)}{\left[\left(\frac{H}{H_{I}}\right)^{2}-\left(\frac{H}{H_{I}}\right)^{n+2}\right]^{\frac{3}{4}}} .
$$

A solução da integral indefinida do lado direito da equação é:

$$
\int \frac{d\left(H / H_{I}\right)}{\left[\left(\frac{H}{H_{I}}\right)^{2}-\left(\frac{H}{H_{I}}\right)^{n+2}\right]^{\frac{3}{4}}}=2\left(\frac{H_{I}}{H}\right)^{\frac{1}{2}} F_{1}\left[\frac{3}{4},-\frac{1}{2 n} ; 1-\frac{1}{2 n} ;\left(\frac{H}{H_{I}}\right)^{n}\right]+\text { cte. }
$$

onde $F_{1}$ é a função hipergeométrica. Nos concentraremos agora no caso $n=2$, pois assim a solução acima pode ser escrita em termos de uma função algébrica,

$$
\phi(H)-\phi_{I}= \pm\left. 2\left(\frac{3 M_{P}^{2} M^{4}}{4 H_{I}^{2}}\right)^{\frac{1}{4}} \cdot\left[\left(\frac{H_{I}}{H}\right)^{2}-1\right]^{\frac{1}{4}}\right|_{H_{I}} ^{H(t)} .
$$

Tomando os limites de integração, obtemos:

$$
\begin{aligned}
& \phi(H)=k\left[\left(\frac{H_{I}}{H}\right)^{2}-1\right]^{\frac{1}{4}}, \\
& H(\phi)=\frac{H_{I}}{\left[1+\sigma^{4} \phi^{4}\right]^{\frac{1}{2}}},
\end{aligned}
$$


onde $k=\left(\frac{12 M_{P}^{2} M^{4}}{H_{I}^{2}}\right)^{\frac{1}{4}}, \sigma=k^{-1}$ e $H(\phi)$ foi obtido tomando a inversa da função que descreve o campo escalar. Aqui, vale ressaltar a semelhança da equação 4.35 com a equação 2.20):

$$
H(a)=\frac{H_{I}}{\sqrt{1+\left(\frac{a}{a_{*}}\right)^{4}}}
$$

onde tomamos $\nu=0, n=2$ e utilizamos a expressão $C=a_{*}^{-4}$. Vemos, por meio de comparação entre as expressões 4.35) e 4.36), que $a \propto \phi$ :

$$
a(\phi)=a_{*}\left(\frac{H_{I}^{2}}{12 M_{P}^{2} M^{4}}\right)^{\frac{1}{4}} \phi .
$$

Assim, conhecendo a expressão para $a(t)$ (Perico et al., 2013), podemos tomar a inversa da equação acima e obter a expressão para $\phi(t)$.

O potencial $V(\phi)$ pode ser obtido substituindo a equação 4.35 em (4.29). Já a densidade de energia da radiação pode ser determinada utilizando as equações (4.28) e (4.29). Com isso, temos:

$$
\begin{aligned}
V(\phi) \equiv \rho_{\Lambda} & =\frac{\rho_{I}}{\left[1+\sigma^{4} \phi^{4}\right]^{2}}, \\
\rho_{r} & =\frac{\rho_{I} \sigma^{4} \phi^{4}}{\left[1+\sigma^{4} \phi^{4}\right]^{2}} .
\end{aligned}
$$

Abaixo, mostramos os gráficos da evolução das densidades e dos parâmetros de densidade da radiação e do vácuo com o campo escalar.
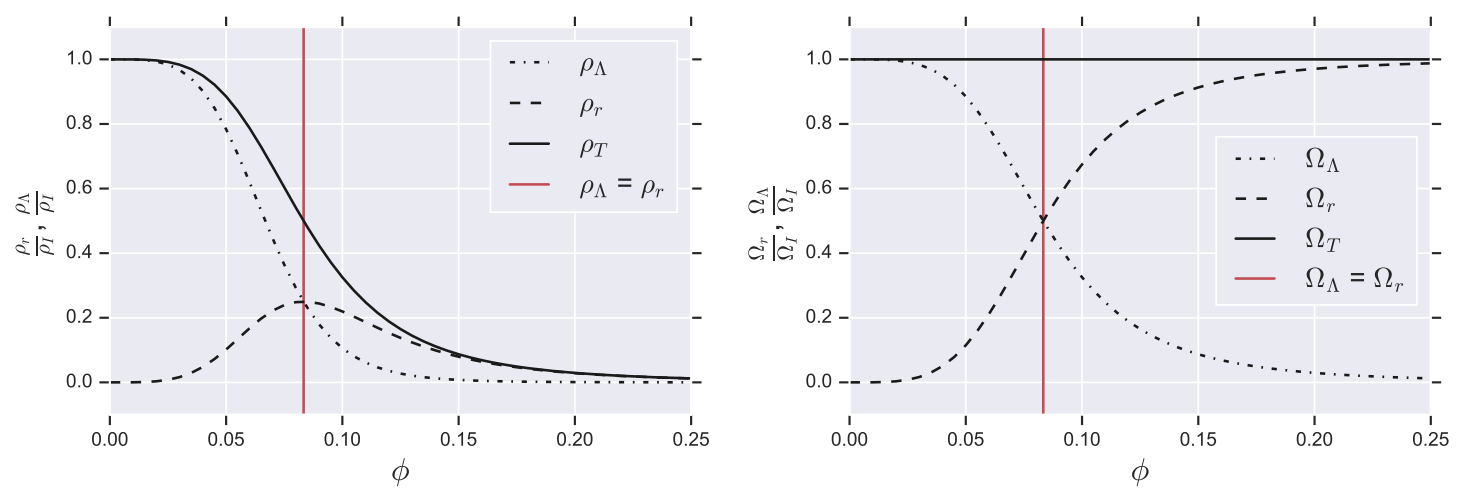

Figura 4.2: No gráfico à esquerda está representada a evolução das densidades com o campo escalar, à direita temos a evolução dos parâmetros de densidade. Aqui, escolhemos $M=M_{P}=H_{I}=1$. A linha vertical em vermelho marca o tempo da igualdade entre as densidades de energia do vácuo e da radiação. Isso marca o fim da Inflação primordial no modelo. 
Esses gráficos mostram que o universo evolui continuamente de uma fase dominada pelo vácuo para uma fase dominada pela radiação. É interessante notar a semelhança entre esses gráficos e os mostrados nas subseções 2.3.1 e 2.4.1. Por esse motivo, uma nova discussão não será feita.

Os resultados demonstrados acima sugerem que a k-essência fornece uma descrição efetiva do decaimento do decaimento do vácuo tal como descrita nos modelos fenomenológicos utilizados na literatura e descritos no capítulo 2 . 
Capítulo 5

\section{Conclusões e Perspectivas}

A discussão abordada nessa dissertação girou em torno de uma classe de modelos com decaimento do vácuo. Sendo assim, é importante frisar os principais resultados que o sugerem como um modelo alternativo viável ao modelo padrão.

Para começar, vamos ressaltar que a lei de decaimento adotada 2.12 nos permite descrever um Universo que começa numa fase de Sitter primordial que evolui naturalmente para a fase da radiação. Ou seja, essa descrição nos fornece uma fase inflacionária primordial sem problema de saída graciosa e livre de horizontes. Além disso, a escala de energia dessa fase pode ser fixada de forma a resolver o problema da constante cosmológica.

Para o Universo tardio o modelo fornece as mesmas transições de fase obtidas no modelo padrão: radiação-matéria-vácuo. O fato de existirem duas transições de fase fluido-vácuo alivia o problema da coincidência cosmológica. Além disso, a descrição da dinâmica do modelo com $\Lambda(t)$ é próxima ao $\Lambda \mathrm{CDM}$, se reduzindo a este quando tomamos $\nu=0$, como seria esperado devido aos resultados observacionais.

O modelo começa e termina em fases de Sitter, estabelecendo uma relação entre a fase inflacionária primordial e o atual período de expansão acelerada, justificando ambos com a densidade de energia do vácuo. Sendo assim, essa classe de modelos oferece algumas vantagens em relação ao modelo padrão.

Essas vantagens justificam uma investigação observacional da viabilidade do modelo. O caminho mais promissor para essa investigação parece ser por meio do espectro das ondas gravitacionais primordiais (Tamayo et al. 2017). Entretanto, para isso é necessário um estudo da fase inflacionária primordial dessa classe de modelos. Com este objetivo, uma descrição de campo escalar para o modelo se torna essencial, visto que essa é a linguagem utilizada para se estudar a inflação. 
Partindo dessa motivação, nós mostramos que, utilizando uma interpretação não tradicional, a k-essência pode descrever o Universo primordial para a classe de modelos com decaimento do vácuo. Essa descrição efetiva recupera toda a dinâmica dos modelos com $\Lambda(t)$ discutidos ao longo do texto. Esse resultado facilitará a descrição do estágio inflacionário primordial e, possivelmente, nos permitirá obter o espectro de potência das ondas gravitacionais primordiais, uma vez que, em geral, esses estão ligados com os parâmetros de rolamento lento (Gron, 2016).

Essa descrição possui, atualmente, duas limitações: não descrever a fase de matéria e não possuir soluções algébricas para $n \neq 2$. Esse primeiro problema significa que, nessa descrição, o modelo não é capaz de resolver o problema da coincidência, já que ele não diz nada à respeito do Universo tardio; é interessante investigar outras escolhas na lagrangeana de Horndeski para resolver essa questão. A segunda limitação é mais uma questão intrigante do que um problema, dado que um estudo detalhado sobre funções hiperbólicas pode fornecer um forma algébrica para o restante dos casos; de qualquer modo é interessante ressaltar a coincidência de que, justamente o caso sustentado por TQC $(n=2)$ possui uma solução algébrica.

Finalmente, é importante fazer um estudo termodinâmico dessa descrição, visto que ela propõe um campo escalar não interagente que descreve tanto a densidade de energia do vácuo quando a densidade de energia do fluido material. 
Apêndices 



\section{Apêndice A}

\section{Tensor Energia-Momento do Vácuo}

Nesse apêndice demonstraremos que a equação de estado do vácuo é dada por $p_{v}=-\rho_{v}$. Seguiremos a linha de raciocínio de Gron (1986).

Todas as propriedades do vácuo devem ser invariantes por transformações de Lorentz $L_{\mu^{\prime}}^{\mu}$, incluindo as componentes do seu tensor energia-momento. Caso contrário o vácuo poderia ser tomado como um referencial privilegiado em relação ao qual observadores inerciais poderiam medir sua velocidade absoluta. Dessa forma, temos:

$$
T_{\mu \nu}=T_{\mu^{\prime} \nu^{\prime}}=L_{\mu^{\prime}}^{\alpha} L_{\nu^{\prime}}^{\beta} T_{\alpha \beta}
$$

Vamos considerar, primeiramente, o caso de observador em um referencial inercial $S$ que observa um sistema $S^{\prime}$ se movimentar ao longo da direção $x$, com velocidade $v$. Nesse caso, a transformação de Lorentz de um sistema para o outro é dada por,

$$
L_{\mu^{\prime}}^{\mu} \equiv\left(\begin{array}{cccc}
\gamma & \gamma^{\nu} & 0 & 0 \\
\gamma^{\nu} & \gamma & 0 & 0 \\
0 & 0 & 1 & 0 \\
0 & 0 & 0 & 1
\end{array}\right),
$$

em que $\gamma=(1-v / c)^{-1 / 2}$ é o fator de contração de Lorentz. Substituindo A.2 em A.1), obtemos:

$$
T_{00}=T_{0^{\prime} 0^{\prime}}=\gamma^{2}\left[T_{00}+v\left(T_{01}+T_{10}\right)+v^{2} T_{11}\right] .
$$

Utilizando o fato de que $\gamma^{2}-1=\gamma^{2} v^{2}$, a equação acima pode ser escrita na forma,

$$
v\left(T_{00}+T_{11}\right)+T_{01}+T_{10}=0 .
$$


A transformação para a componente $T_{11}$ nos fornece o mesmo resultado. De maneira análoga, as transformações para as componentes $T_{01}$ e $T_{10}$ nos dão,

$$
T_{00}+T_{11}+v\left(T_{01}+T_{10}\right)=0
$$

As equações A.4 e A.5 nos fornecem um sistema de equações. Queremos que o sistema seja solucionado para uma velocidade $v$ qualquer. Assim, temos:

$$
T_{00}=-T_{11}, \quad T_{01}=-T_{10} .
$$

As transformações das componentes $T_{02}$ e $T_{12}$ nos fornecem, respectivamente:

$$
\begin{aligned}
& T_{02}=\gamma T_{02}+\gamma v T_{12}, \\
& T_{12}=\gamma v T_{02}+\gamma T_{12},
\end{aligned}
$$

que impõe:

$$
T_{02}=T_{12}=0
$$

Analogamente, encontramos:

$$
T_{20}=T_{21}=T_{03}=T_{13}=T_{30}=T_{31}=0 .
$$

Até o momento, encontramos a seguinte forma para o tensor de energia-momento do vácuo:

$$
T_{\mu \nu}=\left(\begin{array}{cccc}
T_{00} & T_{01} & 0 & 0 \\
-T_{01} & -T_{00} & 0 & 0 \\
0 & 0 & T_{22} & T_{23} \\
0 & 0 & T_{32} & T_{33}
\end{array}\right)
$$

Para obter as restrições sobre as componentes restantes, vamos analisar as transformações de Lorentz para translações nas direções $y$ e $z$. A primeira nos permite inferir:

$$
T_{01}=T_{10}=T_{23}=T_{32}=0, \quad T_{22}=-T_{00} .
$$


Enquanto as translações na direção $z$, implicam

$$
T_{33}=-T_{00}
$$

Substituindo A.12 e A.13 no tensor energia-momento do vácuo A.11), obtemos sua forma final:

$$
T_{\mu \nu}=\left(\begin{array}{cccc}
T_{00} & 0 & 0 & 0 \\
0 & -T_{00} & 0 & 0 \\
0 & 0 & -T_{00} & 0 \\
0 & 0 & 0 & -T_{00}
\end{array}\right) .
$$

Dada essa forma para o tensor energia-momento, podemos encontrar a densidade de energia e pressão para o vácuo:

$$
\begin{aligned}
& \rho_{v}=T_{00} \\
& p_{v}=T_{j}^{i}=-T_{00}
\end{aligned}
$$

Ou seja, podemos associar o vácuo com um fluido perfeito cuja EoS seja dada por $p=-\rho$. 


\section{Apêndice B}

\section{Redshift, Distância de Luminosidade e Horizontes}

As demonstrações desse apêndice seguirão Weinberg (2008a).

\section{B.1 Redshift}

$\mathrm{Na}$ presente seção, vamos demonstrar como o fator de escala pode ser descrito em função do redshift. Para isso, considere a emissão de um fóton por um astro no tempo $t_{1}$. Este fóton é detectado por um observador, à uma distância $r$, no tempo $t_{0}$. Como a luz se movimenta percorrendo uma geodésica no espaço-tempo, seu tempo próprio é nulo, assim na métrica FLRW, podemos escrever:

$$
\begin{aligned}
& d \tau^{2}=d t^{2}-a^{2}(t) \frac{d r^{2}}{1-k r^{2}}=0 \\
& \Rightarrow \frac{d t}{a(t)}=\frac{d r}{\sqrt{1-k r^{2}}}
\end{aligned}
$$

onde descartamos o sinal negativo pois o tempo é definido como estritamente positivo. Agora, podemos integrar essa expressão tomando os limites de integração descritos no parágrafo acima:

$$
\int_{t_{1}}^{t_{0}} \frac{d t}{a(t)}=\int_{0}^{r} \frac{d r^{\prime}}{\sqrt{1-k r^{\prime 2}}} .
$$

A integral do lado direito da equação depende do parâmetro de curvatura do universo (nesse momento não estamos interessados em sua solução, mas ela será importante quando falarmos de distância de luminosidade). Portanto, iremos definí-la como a integral $\mathcal{I}(r)$, cuja solução analítica para cada valor de " $k$ "é dada por: 


$$
\mathcal{I}(r) \equiv \int_{0}^{r} \frac{d r^{\prime}}{\sqrt{1-k r^{\prime 2}}}=\left\{\begin{array}{l}
\operatorname{arcsenh}(r), \quad k=-1 \\
r, \quad k=0 . \\
\operatorname{arcsen}(r), \quad k=1 .
\end{array}\right.
$$

Estabelecida esta notação, a equação $(\overline{B .3})$, pode ser escrita na forma:

$$
\int_{t_{1}}^{t_{0}} \frac{d t}{a(t)}=\mathcal{I}(r) .
$$

Considere, agora, um próximo fóton emitido um tempo $\delta t_{1}$ após o primeiro e detectado um tempo $\delta t_{0}$ após o primeiro. Esses intervalos de tempo podem ser interpretados como o inverso da frequência dos fótons. Assim, podemos escrever:

$$
\int_{t_{1}+\delta t_{1}}^{t_{0}+\delta t_{0}} \frac{d t}{a(t)}=\mathcal{I}(r) .
$$

O caminho percorrido pelos dois fótons pode ser aproximado como tendo sido o mesmo, desde que fonte e observador não estejam se afastando (ou aproximando) com velocidade alta. Em outras palavras, desde que o Universo não esteja se expandindo rapidamente, podemos dizer que os dois fótons percorrem o mesmo caminho. Substituindo (B.6) em (B.5), obtemos:

$$
\begin{array}{r}
\int_{t_{1}+\delta t_{1}}^{t_{0}+\delta t_{0}} \frac{d t}{a(t)}-\int_{t_{1}}^{t_{0}} \frac{d t}{a(t)}=\mathcal{I}(r)-\mathcal{I}(r)=0 \\
\Rightarrow \int_{t_{1}}^{t_{0}} \frac{d t}{a(t)}+\int_{\delta t_{1}}^{\delta t_{0}} \frac{d t}{a(t)}-\int_{t_{1}}^{t_{0}} \frac{d t}{a(t)}=0 \\
\int_{\delta t_{1}}^{\delta t_{0}} \frac{d t}{a(t)}=\frac{\delta t_{1}}{a\left(t_{1}\right)}-\frac{\delta t_{0}}{a\left(t_{0}\right)}=0
\end{array}
$$

Definindo $a\left(t_{i}\right) \equiv a_{i}$, da última igualdade, temos:

$$
\frac{\delta t_{1}}{a_{1}}=\frac{\delta t_{0}}{a_{0}} \Rightarrow \frac{\delta t_{1}}{\delta t_{0}}=\frac{a_{1}}{a_{0}}
$$

Lembrando que os intervalos de tempo $\delta t_{0}$ e $\delta t_{1}$ podem ser pensados como o inverso da frequência dos fótons emitido, podemos escrever

$$
\frac{\nu_{0}}{\nu_{1}}=\frac{a_{1}}{a_{0}} .
$$


O parâmetro de redshift, "z", é definido como a fração da variação do comprimento de onda, $\delta \lambda=\lambda_{0}-\lambda_{1}$, em relação ao comprimento de onda emitido $\lambda_{1}$. Ou seja:

$$
z \equiv \frac{\lambda_{0}-\lambda_{1}}{\lambda_{1}}=\frac{\lambda_{0}}{\lambda_{1}}-1
$$

Agora, utilizamos o resultado sobre a relação entre o comprimento de onda e a frequência de um fóton, $\lambda \nu=c$, para escrever a relação $\mathrm{B} .12$ em termos da frequência

$$
z=\frac{\nu_{1}}{\nu_{0}}-1
$$

Finalmente, substituindo a equação (B.11) em (B.13), podemos escrever o parâmetro de redshift em termos do fator de escala:

$$
z=\frac{a_{0}}{a(t)}-1=\frac{a_{0}-a(t)}{a(t)} .
$$

Onde redefinimos $a_{1} \equiv a(t)$, sem perda de generalidade. Tomando a expressão inversa, podemos escrever o fator de escala em termos do redshift, $a(t) / a_{0}=[1+z(t)]^{-1}$. Este redshift se deve exclusivamente ao da expansão do Universo. No caso de uma medida observacional, deve-se descontar o efeito Doppler correspondente às velocidades peculiares, seja ele de "blueshift" (objetos se aproximando) ou de "redshift" (objetos se afastando).

\section{B.2 Distância de Luminosidade}

Antes de mais nada, é importante definir os conceitos de luminosidade aparente e absoluta. A luminosidade absoluta é definida como a quantidade de energia $\Delta E$ emitida por uma fonte luminosa no intervalo de tempo $\Delta t$,

$$
L \equiv \frac{\Delta E}{\Delta t} .
$$

Já a luminosidade aparente é definida como a quantidade de energia $\Delta E^{\prime}$ que atinge um detector de área $A$ no tempo $\Delta t$ :

$$
l \equiv \frac{\Delta E^{\prime}}{A \Delta t}
$$

Essa quantidade de energia $\Delta E^{\prime}$ é uma fração da quantidade de energia emitida $\Delta E$, que deve ser proporcional à área $A$ do detector e, como a radiação é emitida em todas as 
direções, ela deve ser inversamente proporcional à área de uma esfera centrada na fonte, cujo raio é a distância entre a fonte e observador. É comum denominar esse raio por distância de luminosidade $d_{l}$ :

$$
\Delta E^{\prime}=\frac{A}{4 \pi d_{l}^{2}} \Delta E .
$$

Assim, substituindo (B.17) em (B.16) obtemos a distância de luminosidade,

$$
d_{l}=\sqrt{\frac{L}{4 \pi l}} .
$$

Note que, se existir algum objeto astronômico cuja luminosidade absoluta é conhecida, podemos utilizar a medida da sua luminosidade aparante para determinar a distância que aquele objeto se encontra. Na verdade, em astronomia o usual é medir a magnitude de um objeto, mas essas suas grandezas se relacionam por meio da equação,

$$
M_{b o l, *}-M_{b o l, \odot}=-2.5 \log _{10}\left(\frac{L_{*}}{L \odot}\right)
$$

onde o subíndice $\odot$ indica uma grandeza solar e "** indica uma grandeza do objeto observado.

Utilizando a hipótese de que a fonte emite, isotropicamente, $n$ fótons de frequência $\delta \nu_{1}$ no intervalo de tempo $\delta t_{1}$, podemos obter a reescrever a luminosidade absoluta B.15 desse objeto:

$$
L=\frac{\Delta E}{\Delta t}=\frac{n h \nu_{1}}{\delta t_{1}}
$$

onde h é a constante de Planck. Os fótons nos atingem com uma frequência $\nu_{0}$ em no intervalo de tempo $\delta t_{0}$. Assim, a luminosidade aparente é dada por:

$$
l=\frac{f \Delta E}{A \Delta t}=f \frac{n h \nu_{0}}{A \delta t_{0}},
$$

onde utilizamos a igualdade (B.17). Como dissemos anteriormente, a fração $f$ deve ser inversamente proporcional à área de uma esfera centrada na fonte, cujo raio $r$ é a distância entre a fonte e o observador. Levando em conta que o Universo está se expandindo, essa distância não é constante durante o tempo que o fóton leva para viajar da fonte até o observador, dessa forma, precisamos obter a área levando isso em conta. Utilizando a métrica FLWR em coordenadas esféricas, temos, 


$$
S=\int_{0}^{\pi} \int_{0}^{2 \pi} \sqrt{g_{\theta \theta}} \sqrt{g_{\phi \phi}} d \theta d \phi=4 \pi a^{2}(t) r^{2}
$$

onde $g_{\theta \theta}$ e $g_{\phi \phi}$ são determinados pela métrica:

$$
g_{\theta \theta}=a^{2}(t) r^{2}, g_{\phi \phi}=a^{2}(t) r^{2} \operatorname{sen}^{2} \theta .
$$

Substituindo essa área na fração $f$ da relação B.21 e, dividindo B.20 por ela, obtemos,

$$
\frac{L}{l}=\frac{\nu_{1} \delta t_{0}}{\nu_{0} \delta t_{1}} 4 \pi a_{0}^{2} r^{2}
$$

Utilizando as relações B.10 e B.11 e, dividindo a equação acima por $4 \pi$, podemos escrever a equação acima na forma,

$$
\frac{L}{4 \pi l}=\frac{a_{0}^{4}}{a_{1}^{2}} r^{2} .
$$

Substituindo (B.25) em (B.18), obtemos a distância de luminosidade em função do fator de escala,

$$
d_{L}=\frac{a_{0}^{2}}{a(t)} r
$$

onde utilizamos $a(t)$ no lugar de $a_{1}$ para indicar que esse resultado vale para um tempo $t$ qualquer, podemos fazer isso pois bastaria redefinir $t_{1} \equiv t$ sem nenhuma perda de generalidade. Finalmente, podemos reescrever essa distância em termos do redshift por meio da expressão (B.14):

$$
d_{L}(z)=(1+z) a_{0} r(z)
$$

Ainda falta um último passo para que possamos obter a expressão exata para o modelo $\Lambda$ CDM plano em termos de medidas observacionais: precisamos obter $r(z)$ em função dos parâmetros de densidade do modelo. Utilizando as expressões (B.3) e (B.4) podemos tomar sua inversa de $\mathcal{I}(\mathrm{r})$ para obter,

$$
r(z)=F\left[\int_{t(z)}^{t_{0}} \frac{d t}{a(t)}\right],
$$


onde utilizamos a notação $F[y]$ para indicar que $F$ é uma função do argumento entre colchetes. Essa função depende do parâmetro de curvatura $k$, sendo:

$$
\mathcal{I}(r) \equiv \int_{0}^{r} \frac{d r^{\prime}}{\sqrt{1-k r^{\prime 2}}}=\left\{\begin{array}{l}
\operatorname{senh}(y), \quad k=-1 \\
y, \quad k=0 \\
\operatorname{sen}(y), \quad k=1 .
\end{array}\right.
$$

Para o caso do modelo padrão temos $k=0$ e podemos obter a integral dentro dos colchetes em termos dos parâmetros de densidade por meio da equação (1.62):

$$
r(z)=\frac{1}{a_{0} H_{0}} \int_{\frac{1}{1+z}}^{1} \frac{d x}{x^{2} \sqrt{\Omega_{\Lambda 0}+\Omega_{m 0} x^{-3}+\Omega_{r 0} x^{-4}}} .
$$

Agora, basta substituir a expressão acima em (B.27) para obter a expressão da distância de luminosidade no modelo $\Lambda \mathrm{CDM}$,

$$
d_{L}(z)=\frac{(1+z)}{H_{0}} \int_{\frac{1}{1+z}}^{1} \frac{d x}{x^{2} \sqrt{\Omega_{\Lambda 0}+\Omega_{m 0} x^{-3}+\Omega_{r 0} x^{-4}}} .
$$

Como foi dito anteriormente, a medida de distância luminosa pode ser obtida observacionalmente se soubermos qual é a luminosidade absoluta do objeto observado. Assim, a integral acima pode ser utilizada para se determinar os parâmetros de densidade observacionalmente, uma vez que de possa de várias medidas de distância de luminosidade é possível fazer um estudo estatístico para obter os parâmetros de densidade que melhor ajustam todas as distâncias.

De fato, existem objetos cuja luminosidade absoluta pode ser determinada. Alguns dos exemplos mais comuns são as Cefeidas e as Supernovas do tipo Ia (Carroll e Ostlie, 2007), por esse motivo, esses objetos são comumente chamados de velas padrão do Universo. As primeiras, são estrelas de luminosidade variável com período conhecido, sendo assim a determinação observacional desse período fornece a magnitude absoluta dessa estrela, nos permitindo determinar sua distância. Esses objetos, foram utilizados por Hubble na sua determinação original de $H_{0}$, elas são utilizadas para escalas da ordem de $\approx 20 M p c$. As supernovas ocorrem quando anãs brancas em um sistema binário acretam suficiente matéria da sua parceira para ultrapassar o limite de Chandrasekhar: a massa máxima que pode ser suportada pela pressão de degenerescência do núcleo estelar. Quando isso acontece, pode ser observada, a vários parsecs de distância, uma explosão da anã branca 
instável. Devido ao fato de que essa explosão ocorre quando as estrelas possuem a massa do limite de Chandrasekhar, sua magnitude absoluta é sempre a mesma, sendo de 4 a 6 ordens de grandeza maior do que a magnitude de estrelas comuns; o que nos permite identificar sua ocorrência em outras galáxias. Assim, podemos utilizar supernovas para obter a distância de luminosidade para as galáxias hospedeiras destes eventos e, com suficiente medidas, determinar os parâmetros de densidade com um elevado grau de confiança. A sua observação nos permite obter informações para escalas da ordem de $\approx 100 M p c$.

\section{B.3 Horizontes}

Horizontes podem ser pensados como uma superfície limite que separa duas regiões com comportamentos diferentes. O exemplo mais comum de horizonte que temos é o horizonte de eventos de um buraco negro: para distâncias menores do que a do horizonte de eventos, nenhum objeto consegue escapar da atração gravitacional do buraco negro. Em outras palavras, o horizonte de eventos delimita a região em que um observador é capaz de receber um sinal sobre uma fonte qualquer.

Em cosmologia, existem dois tipos de horizontes que nos interessam, o horizonte de eventos e o horizonte de partículas. Já definimos o primeiro deles no parágrafo acima, portanto nos resta definir o conceito de horizonte de partículas: a distância no tempo atual para que dois objetos estivessem em contato causal no tempo $t_{e}$. Ou seja, enquanto o primeiro define a distância máxima que uma fonte pode ter nós para que sejamos capazes de receber seu sinal em algum momento do futuro, o segundo define qual a distância máxima que um objeto pode ter de nós atualmente para que estivéssemos em contato causal em algum tempo do passado. Vamos estudar, à seguir, as condições cosmológicas para que cada um desses horizontes aconteça.

\section{B.3.1 Horizonte de Eventos}

Um horizonte de eventos ocorrerá na métrica FLRW se a taxa de expansão do Universo, $H(t)$, for suficientemente alta, de forma que fótons partindo de uma distância $r>r_{H E}$, onde $r_{H E}$ é a distância do horizonte, levem um tempo infinito para atingir um observador em $r=0$. Isso significa que, de acordo com a definição dada no parágrafo anterior, eventos que ocorram em $r>r_{H E}$ estarão, para sempre, inacessíveis ao observador. Podemos 
utilizar a equação (B.3), com os limites adequados, para descrever a trajetória desse fóton,

$$
\int_{t_{0}}^{\infty} \frac{d t^{\prime}}{a\left(t^{\prime}\right)}=\int_{0}^{r_{H E}} \frac{d r}{\sqrt{1-k r^{2}}}
$$

Vamos resolver apenas para o caso $k=0$, dado que queremos obter a distância do horionte de eventos para o modelo $\Lambda \mathrm{CDM}$ plano. A distância própria do horizonte de eventos é dada por,

$$
d_{H E}=\int_{0}^{r_{H E}} \sqrt{g_{r r}} d r=a_{0} \int_{0}^{r_{H E}} \frac{d r}{\sqrt{1-k r^{2}}} .
$$

Assim, podemos substituir B.33 em B.32, para obter a distância do horizonte de eventos:

$$
d_{H E}=a_{0} \int_{t_{0}}^{\infty} \frac{d t}{a(t)} .
$$

Para resolver a integral do lado direito, basta utilizarmos a expressão para $a(t)$ do modelo $\Lambda$ CDM (1.60). Com isso, obtemos,

$$
d_{H E}=a_{0} \int_{t_{0}}^{\infty} \frac{1}{a_{0}}\left[\frac{3(1+\omega) H_{0} t}{2}\right]^{-2 / 3(1+\omega)} d t .
$$

Efetuando a integração acima, obtemos:

$$
d_{H E}=\left.\left[\frac{3(1+\omega) H_{0}}{2}\right]^{-2 / 3(1+\omega)} t^{(3 \omega+1) / 3(\omega+1)}\right|_{t_{0}} ^{\infty} .
$$

O horizonte de eventos existirá apenas quando a integral acima não divergir. Para que isso aconteça, o expoente de $t$ deve ser negativo. Como $1+\omega \geq 0$ para todos os valores permitidos de $\omega$, queremos:

$$
\begin{aligned}
& 3 \omega+1<0, \\
& \Rightarrow \omega<-\frac{1}{3} .
\end{aligned}
$$

Portanto, vemos que o horizonte de eventos só existe para universos cuja dinâmica é dominada por um fluido com $\omega<-\frac{1}{3}$, ou seja, um universo acelerado. 


\section{B.3.2 Horizonte de Partículas}

Matematicamente, as diferenças nas definições dos dois tipos de horizontes de traduzem nos limites da integral (B.34), relembrando que o horizonte de partículas nos dá a distância máxima que dois objetos podem ter atualmente para terem estado em contato causal no tempo $t_{e}$. Vamos tomar $t_{e} \rightarrow 0$, para analisar dois fótons vindos da época do Big Bang:

$$
d_{H P}=a_{0} \int_{0}^{t_{0}} \frac{d t}{a(t)} .
$$

Podemos resolver essa equação da mesma maneira que fizemos para o horizonte de partículas, basta substituir a expressão para $a(t)$ 1.60. A solução dessa integral é exatamente a fornecida pela equação $(\mathrm{B} .36)$, com os limites de integração adequados,

$$
d_{H P}=\left.\left[\frac{3(1+\omega) H_{0}}{2}\right]^{-2 / 3(1+\omega)} t^{(3 \omega+1) / 3(\omega+1)}\right|_{0} ^{t_{0}} .
$$

Assim como para o horizonte de eventos, o horizonte de partículas só existirá quando a integral acima convergir. Nesse caso, o expoente de t não pode ser negativo, portanto queremos,

$$
\begin{aligned}
& 3 \omega+1 \geq 0, \\
& \Rightarrow \omega \geq-\frac{1}{3} .
\end{aligned}
$$

A igualdade foi incluída na condição acima, pois a integral converge nesse caso. Repare que as condições (B.38) e (B.42) são complementares, ou seja, é impossível que um cenário cosmológico esteja livre de ambos os horizontes. A condição (B.42 mostra que universos acelerados estão livres de horizonte de partículas. Com isso, podemos afirmar que um Universo do tipo de Sitter é livre de horizonte de partículas, mas possui um horizonte de eventos. 


\section{Referências Bibliográficas}

Abdel-Rahman A-M. M., Singularity-free decaying-vacuum cosmologies, Phys. Rev. D, 1992, vol. 45, p. 3497 , doi:10.1103/PhysRevD.45.3497.

Aghanim N., et al., Planck 2018 results. VI. Cosmological parameters, 2018 , arXiv:1807.06209.

Alpher R. A., Bethe H., Gamow G., The Origin of Chemical Elements, Phys. Rev., 1948, vol. 73 , p. 803 , doi:10.1103/PhysRev.73.803.

Alpher R. A., Herman R. C., Evolution of the Universe. vol. 162, Nature, 1948

Amanullah R., et al., Spectra and Light Curves of Six Type Ia Supernovae at 0.511 ; z ; 1.12 and the Union2 Compilation, Astrophys. J., 2010, vol. 716, p. 712 , doi:10.1088/0004$637 \mathrm{X} / 716 / 1 / 712$.

Armendariz-Picon C., Damour T., Mukhanov V. F., k - inflation, Phys. Lett., 1999, vol. B458, p. 209 , arXiv:hep-th/9904075.

Armendariz-Picon C., Mukhanov V., Steinhardt P. J., Essentials of k-essence, Physical Review D, 2001, vol. 63 , doi:10.1103/physrevd.63.103510.

Basilakos S., Plionis M., Solà J., Hubble expansion \& Structure Formation in Time Varying Vacuum Models, Phys. Rev., 2009, vol. D80, p. 083511 , arXiv:0907.4555.

Berera A., Warm inflation, Phys. Rev. Lett., 1995, vol. 75, p. 3218 , arXiv:astro$\mathrm{ph} / 9509049$.

Bergmann P. G., Comments on the scalar tensor theory, Int. J. Theor. Phys., 1968, vol. 1, p. 25 , doi:10.1007/BF00668828. 
Blumenthal G. R., Faber S. M., Primack J. R., Rees M. J., Formation of galaxies and large-scale structure with cold dark matter., Nature, 1984, vol. 311, p. 517 , doi:10.1038/311517a0.

Carneiro S., Tavakol R., On vacuum density, the initial singularity and dark energy, Gen. Rel. Grav., 2009, vol. 41, p. 2287 , arXiv:0905.3131. [Int. J. Mod. Phys.D18,2343(2009)]

Carroll B. W., Ostlie D. A., An Introduction to Modern Astrophysics 2nd (international) edn. Addison-Wesley, San Francisco: Pearson, 2007

Carroll S. M., Spacetime and Geometry. Cambridge University Press, 2004 , URL http: //www.slac.stanford.edu/spires/find/books/www?cl=QC6:C37:2004.

Carvalho J. C., Lima J. A. S., Waga I., On the cosmological consequences of a time dependent lambda term, Phys. Rev., 1992, vol. D46, p. 2404 , doi:10.1103/PhysRevD.46.2404.

Chen W., Wu Y.-S., Implications of a cosmological constant varying as, Physical Review D, 1990, vol. 41, p. 695 , doi:10.1103/physrevd.41.695.

Chiba T., Dutta S., Scherrer R. J., Slow-roll k-essence, Phys. Rev., 2009, vol. D80, p. 043517 , arXiv:0906.0628.

Clifton T., Ferreira P. G., Padilla A., Skordis C., Modified Gravity and Cosmology, Phys. Rept., 2012, vol. 513, p. 1 , arXiv:1106.2476.

Clowe D., Bradac M., Gonzalez A. H., Markevitch M., Randall S. W., Jones C., Zaritsky D., A direct empirical proof of the existence of dark matter, Astrophys. J. Lett., 2006, vol. 648, p. L109, arXiv:astro-ph/0608407.

de Sitter W., On the relativity of inertia. Remarks concerning Einstein's latest hypothesis, Koninklijke Nederlandse Akademie van Wetenschappen Proceedings Series B Physical Sciences, 1917, vol. 19, p. 1217

Dicke R. H., Peebles P. J. E., Roll P. G., Wilkinson D. T., Cosmic Black-Body Radiation, Astrophys. J., 1965, vol. 142, p. 414 , doi:10.1086/148306.

Dodelson S., Modern cosmology 1 edn. Academic Press, 2003 , URL http://gen.lib. rus.ec/book/index .php?md5=a58cc844174b751d6bcfeee415a948b2 . 
Einstein A., On the electrodynamics of moving bodies, Annalen Phys., 1905, vol. 17, p. 891 , doi:10.1002/andp.200590006. [Annalen Phys.14,194(2005)]

Einstein A., The Field Equations of Gravitation, Sitzungsber. Preuss. Akad. Wiss. Berlin (Math. Phys.), 1915, vol. 1915, p. 844

Einstein A., Cosmological Considerations in the General Theory of Relativity, Sitzungsber. Preuss. Akad. Wiss. Berlin (Math. Phys.), 1917, vol. 1917, p. 142

Eisenstein D. J., et al., Detection of the Baryon Acoustic Peak in the Large-Scale Correlation Function of SDSS Luminous Red Galaxies, Astrophys. J., 2005, vol. 633, p. 560 , arXiv:astro-ph/0501171.

Friedmann A., On the Curvature of space, Z. Phys., 1922, vol. 10, p. 377 , doi:10.1007/BF01332580. [Gen. Rel. Grav.31,1991(1999)]

Friedmann A., On the Possibility of a world with constant negative curvature of space, Z. Phys., 1924, vol. 21, p. 326 , doi:10.1007/BF01328280. [Gen. Rel. Grav.31,2001(1999)]

Gamow G., The Evolutionary Universe, Scientific American, 1956, vol. 195, p. 136 , doi:10.1038/scientificamerican0956-136.

Grande J., Sola J., Basilakos S., Plionis M., Hubble expansion and structure formation in the 'running FLRW model' of the cosmic evolution, JCAP, 2011, vol. 1108, p. 007 , arXiv:1103.4632.

Gron O., Repulsive Gravitation and Inflationary Universe Models, Am. J. Phys., 1986, vol. 54, p. 46 , doi:10.1119/1.14769.

Gron O., Warm Inflation, Universe, 2016, vol. 2, p. 20 , doi:10.3390/universe2030020.

Guth A. H., Inflationary universe: A possible solution to the horizon and flatness problems, Physical Review D, 1981, vol. 23 , doi:10.1103/physrevd.23.347.

Guth A. H., Weinberg E. J., Could the universe have recovered from a slow first-order phase transition?, Nuclear Physics B, 1983, vol. 212 , doi:10.1016/0550-3213(83)90307-3.

Hawking S. W., Moss I. G., Stewart J. M., Bubble collisions in the very early universe,

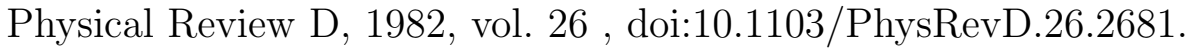


Hinshaw G., et al., Nine-Year Wilkinson Microwave Anisotropy Probe (WMAP) Observations: Cosmological Parameter Results, Astrophys. J. Suppl., 2013, vol. 208, p. 19 , arXiv:1212.5226.

Horndeski G. W., Second-order scalar-tensor field equations in a four-dimensional space, Int. J. Theor. Phys., 1974, vol. 10, p. 363 , doi:10.1007/BF01807638.

Hubble E., A relation between distance and radial velocity among extra-galactic nebulae, Proc. Nat. Acad. Sci., 1929a, vol. 15, p. 168 , doi:10.1073/pnas.15.3.168.

Hubble E., A spiral nebula as a stellar system, Messier 31., ApJ, 1929b, vol. 69, p. 103 , doi:10.1086/143167.

Kamionkowski M., Kovetz E. D., The Quest for B Modes from Inflationary Gravitational Waves, Annual Review of Astronomy and Astrophysics, 2016, vol. 54, p. 227 , arXiv:1510.06042.

Kase R., Tsujikawa S., Dark energy in Horndeski theories after GW170817: A review, Int. J. Mod. Phys., 2019, vol. D28, p. 1942005 , arXiv:1809.08735.

Kirshner R. P., Hubble's diagram and cosmic expansion, Proceedings of the National Academy of Science, 2004, vol. 101, p. 8 , doi:10.1073/pnas.2536799100.

Kolb E. W., Turner M. S., The early universe. Frontiers in Physics, Addison-Wesley Publishing Company, 1990 , URL http://gen.lib.rus.ec/book/index.php?md5= 50e1df2ef0896df989baffd78baf6d57.

Landau L. D., Lifshitz E. M., The classical theory of fields 4 edn. vol. Volume 2, Butterworth Heinemann, 1980 , URL http://gen.lib.rus.ec/book/index.php?md5= d17e5c15636bc940268c26682f10a459.

Lemaitre G., The Beginning of the World from the Point of View of Quantum Theory., Nature, 1931, vol. 127, p. 706 , doi:10.1038/127706b0.

Liddle A. R., Lyth D. H., Cosmological inflation and large-scale structure 1st edn. Cambridge University Press, 2000 , URL http://gen.lib.rus.ec/book/index.php?md5= db2775b127da7183e1b18e1c1f520df5. 
Lima J., Basilakos S., Solà J., Thermodynamical aspects of running vacuum models, Eur. Phys. J. C, 2016, vol. 76, p. 228 , arXiv:1509.00163.

Lima J. A. S., Almeida P. E. M., . K-essence and Decaying Vacuum. a ser submetido

Lima J. A. S., Basilakos S., Solà J., Expansion History with Decaying Vacuum: A Complete Cosmological Scenario, Mon. Not. Roy. Astron. Soc., 2013, vol. 431, p. 923 , arXiv:1209.2802.

Lima J. A. S., Germano A. S. M., On the equivalence of bulk viscosity and matter creation, Physics Letters A, 1992, vol. 170, p. 373 , doi:10.1016/0375-9601(92)90890-X.

Lima J. A. S., Maia J. M. F., Deflationary cosmology with decaying vacuum energy density, Phys. Rev., 1994, vol. D49, p. 5597 , doi:10.1103/PhysRevD.49.5597.

Lima J. A. S., Santos R. C., Do Eclipse Solar de 1919 ao Espetáculo das Lentes Gravitacionais, Revista Brasileira de Ensino de Física, 2019, vol. 41, URL http://www. scielo. br/scielo.php?script=sci_arttext\&pid=S1806-11172019000500204\&nrm=iso.

Lima J. A. S., Trodden M., Decaying vacuum energy and deflationary cosmology in open and closed universes, Phys. Rev. D, 1996, vol. 53, p. 4280 , arXiv:astro-ph/9508049.

Maia J. M. F., . Algumas Aplicações de Campos Escalares em Cosmologia. PhD thesis Instituto de Física - Universidade de São Paulo (IFUSP)

Maia J. M. F., Lima J. A. S., Scalar field description of decaying- $\Lambda$ cosmologies, Phys. Rev. D, 2002, vol. 65, p. 083513 , arXiv:astro-ph/0112091.

Mukhanov V., Physical Foundations of Cosmology. Cambridge University Press Oxford, 2005

Mukhanov V. F., Vikman A., Enhancing the tensor-to-scalar ratio in simple inflation, JCAP, 2006, vol. 0602, p. 004 , arXiv:astro-ph/0512066.

Nemiroff R., Bonnell J., The Nature of the Universe Debate in 1998, Publications of the Astronomical Society of the Pacific, 1999, vol. 111, p. 285, URL http://www.jstor. org/stable/10.1086/316326. 
Ozer M., Taha M. O., A Solution to the Main Cosmological Problems, Phys. Lett., 1986, vol. B171, p. 363 , doi:10.1016/0370-2693(86)91421-8.

Ozer M., Taha M. O., A Model of the Universe with Time Dependent Cosmological Constant Free of Cosmological Problems, Nucl. Phys., 1987, vol. B287, p. 776 , doi:10.1016/0550-3213(87)90128-3.

Peebles P. J. E., Large-scale background temperature and mass fluctuations due to scaleinvariant primeval perturbations, ApJ, 1982, vol. 263, p. L1 , doi:10.1086/183911.

Peebles P. J. E., Principles of physical cosmology. Princeton University Press, 1994

Penzias A. A., Wilson R. W., A Measurement of Excess Antenna Temperature at 4080 Mc/s., ApJ, 1965, vol. 142, p. 419 , doi:10.1086/148307.

Perico E. L. D., Lima J. A. S., Basilakos S., Solà J., Complete Cosmic History with a dynamical $\Lambda=\Lambda(H)$ term, Phys. Rev., 2013, vol. D88, p. 063531, arXiv:1306.0591.

Perlmutter S., et al., Measurements of $\Omega$ and $\Lambda$ from 42 High-Redshift Supernovae, The Astrophysical Journal, 1999, vol. 517, p. 565 , doi:10.1086/307221.

Poincaré H., La dynamique de l'électron, Revue générale des sciences pures et appliquées, 1908, vol. 19, p. 386

Prigogine I., Geheniau J., Gunzig E., Nardone P., Thermodynamics and cosmology, General Relativity and Gravitation, 1989, vol. 21 , doi:10.1007/BF00758981.

Ratra B., Peebles P. J. E., Cosmological Consequences of a Rolling Homogeneous Scalar Field, Phys. Rev., 1988, vol. D37, p. 3406 , doi:10.1103/PhysRevD.37.3406.

Riess A. G., et al., Observational evidence from supernovae for an accelerating universe and a cosmological constant, Astron. J., 1998, vol. 116, p. 1009 , arXiv:astro-ph/9805201.

Riess A. G., Macri L., Casertano S., Lampeitl H., Ferguson H. C., Filippenko A. V., Jha S. W., Li W., Chornock R., A 3\% Solution: Determination of the Hubble Constant with the Hubble Space Telescope and Wide Field Camera 3, Astrophys. J., 2011, vol. 730, p. 119 , arXiv:1103.2976. [Erratum: Astrophys. J.732,129(2011)] 
Robertson H. P., Kinematics and World-Structure, Astrophys. J., 1935, vol. 82, p. 284 , doi:10.1086/143681.

Rubin V. C., Ford W. K. J., Thonnard N., Rotational properties of 21 SC galaxies with a large range of luminosities and radii, from NGC $4605(\mathrm{R}=4 \mathrm{kpc})$ to UGC 2885 $(\mathrm{R}=122 \mathrm{kpc}) .$, ApJ, 1980, vol. 238, p. 471, doi:10.1086/158003.

Santos J. R. L., Moraes P. H. R. S., Cosmology from a running vacuum model driven by a scalar field, International Journal of Geometric Methods in Modern Physics, 2018 , arXiv:1806.07493.

Schneider P., Extragalactic Astronomy and Cosmology: An Introduction 1 edn. Elsevier, 2006 , URL http://gen.lib.rus.ec/book/index.php?md5= a983dff228c9f489c7eaef5b25d024f5.

Sebastiani L., Myrzakul S., Myrzakulov R., Reconstruction of k-essence inflation in Horndeski gravity, Eur. Phys. J. Plus, 2017, vol. 132, p. 433 , arXiv:1702.00064.

Shapiro I. L., Solà J., The scaling evolution of the cosmological constant, Journal of High Energy Physics, 2002, vol. 2002, p. 006 , doi:10.1088/1126-6708/2002/02/006.

Shapiro I. L., Solà J., In Theoretical and experimental problems of general relativity and gravitation. Gravity, strings and quantum field theory. Proceedings, 11th Conference and International Workshop, GRG 11, Tomsk, Russia, July 1-8, 2002 , 2002, p. 1238 , arXiv:hep-ph/0210329., URL http://www-library.desy.de/preparch/desy/proc/ proc02-02/Proceedings/pa.4/sola_pr.pdf. . [,1238(2002)]

Smoot G. F., et al., Structure in the COBE differential microwave radiometer first year maps, Astrophys. J., 1992, vol. 396, p. L1, doi:10.1086/186504.

Solà J., Dark energy: A Quantum fossil from the inflationary Universe?, J. Phys., 2008, vol. A41, p. 164066 , arXiv:0710.4151.

Starobinsky A. A., A New Type of Isotropic Cosmological Models Without Singularity, Phys. Lett., 1980, vol. 91B, p. 99 , doi:10.1016/0370-2693(80)90670-X. [Adv. Ser. Astrophys. Cosmol.3,130(1987); ,771(1980)] 
Sunyaev R. A., Zeldovich Ya. B., Small scale fluctuations of relic radiation, Astrophys. Space Sci., 1970, vol. 7, p. 3

Sunyaev R. A., Zeldovich Ya. B., Microwave background radiation as a probe of the contemporary structure and history of the universe, Ann. Rev. Astron. Astrophys., 1980, vol. 18, p. 537 , doi:10.1146/annurev.aa.18.090180.002541.

Tamayo D. A., Lima J. A. S., Alves M. E. S., de Araujo J. C. N., Primordial Gravitational Waves in Running Vacuum Cosmologies, Astropart. Phys., 2017, vol. 87, p. 18 , arXiv:1504.07092.

Unnikrishnan S., Sahni V., Toporensky A., Refining inflation using non-canonical scalars, JCAP, 2012, vol. 1208, p. 018 , arXiv:1205.0786.

Vikman A., . K-essence: cosmology. PhD thesis Ludwig-Maximilians-Universität München

Walker A. G., On Riemannian Spaces with Spherical Symmetry about a Line, and the Conditions for Isotropy in General Relativity, The Quarterly Journal of Mathematics, 1935, vol. os-6, p. 81 , doi:10.1093/qmath/os-6.1.81.

Weinberg S., The Cosmological Constant Problem, Rev. Mod. Phys., 1989, vol. 61, p. 1 , doi:10.1103/RevModPhys.61.1. [,569(1988)]

Weinberg S., Cosmology. Cosmology, OUP Oxford, 2008a, URL https://books.google. com.br/books?id=nqQZdg020fsC.

Weinberg S., Effective field theory for inflation, Phys. Rev. D, 2008b, vol. 77, p. 123541 , doi:10.1103/PhysRevD.77.123541.

Wu X.-P., Chiueh T., Fang L.-Z., Xue Y.-J., A comparison of different cluster mass estimates: consistency or discrepancy?, Mon. Not. Roy. Astron. Soc., 1998, vol. 301, p. 861 , arXiv:astro-ph/9808179.

Yang W., Banerjee N., Pan S., Constraining a dark matter and dark energy interaction scenario with a dynamical equation of state, Phys. Rev., 2017, vol. D95, p. 123527 , arXiv:1705.09278. [Addendum: Phys. Rev.D96,no.8,089903(2017)] 
Zilioti G. J. M., Santos R. C., Lima J. A. S., From de Sitter to de Sitter: decaying vacuum models as a possible solution to the main cosmological problems, Adv. High Energy Phys., 2018, vol. 2018, p. 6980486 , arXiv:1508.06344. 